\title{
REAL-TIME PROCESSING OF BINARY IMAGES FOR INDUSTRIAL APPLICATIONS
}

\author{
Foith, J.P., Eisenbarth, C., Enderle, E., Geisselmann, H., \\ Ringshauser, H., Zimmermann, G. \\ Fraunhofer-Institut für Informations- und Datenverarbeitung \\ Karlsruhe, W-Germany
}

\begin{abstract}
Summary
This paper deals with problems and solutions in applications of Image Analysis Systems. Important applications are visual inspection, sensor-controlled handling and assembly, and the control of tools, machines, or processes. When designing Image Analysis Systems one must not only solve image analys is problems: the whole workplace must be engineered in order to reach practical solutions.

One particular requirement for Image Analysis Systems is the capability of real-time processing. Selected methods of image processing and analys is are discussed under this aspect. It is pointed out why practical systems perform binary image processing, and it is argued that this suffices for many practical tasks. A brief survey of existing systems shows the state-of-the-art and a discussion of applications illustrates the power of these systems.

In the third part is presented a system -- 'S.A.M.' (for: Sensorsystem for Automation and Measurement) -- that the authors have developed. S.A.M. is a kit whose modules can be combined into configurations that are adapted to particular tasks. S.A.M. hardware consists of three groups of modules that perform: 1) video input processing, 2) image processing and analys is (including image storage), and 3) data processing. The purpose of the second group of processors is the reduction of data (TV on-line feature extraction). Extracted features are: component labels, area, perimeter, number of holes, and centroid coordinates for up to 255 blobs in a binary image. These data are collected and highly organized by the Data Processing Modules. S.A.M. software is implemented in three layers: 1) basic software, 2) problem oriented software, and 3) operator oriented software. With the aid of S.A.M. hardware and software the analysis of complex binary images can be performed in less than $500 \mathrm{~ms}$-- despite the use of a micro-processor. In concluding, the authors give an example where a S.A.M. configuration was coupled with an industrial robot for grasping parts from a running conveyor belt.
\end{abstract}


1. DIGITAL IMAGE ANALYSIS SYSTEMS FOR INDUSTRIAL APPLICATIONS

The present economic times are marked by high costs for labor and material, lack of trained workers, avoidance of inhumane work, demands of reliability, safety, and quality, as well as aspects of environmental protection. In the last years, industrial nations have put a lot of effort into the development of more efficient productivity technologies in order to cope with that situation. Improvements in this area appeal for social, economic, and technological reasons.

Manufacturing operations are generally classified as either mass production, batch production, or job shop /ABRAHAM et al. 177/. While mass production is automated since long, this did not hold for batch manufacturing until a decade ago. With the advent of industrial robot technologies, we are about to experience improvements in batch production that will continue to increase within the next two decades /COLDING et a1. 179/. In the early days of research and development in robotics technology emphasis was on manipulator arms and control algorithms. In the meantime it has become obvious that these manipulator systems must be equipped with sensors in order to be applicable in a wide variety of tasks. Sensor systems can be based on many different physical signals. In robotics mostly visual and/or tactile sensor systems are put to use. In this paper we will only deal with visual sensor systems.

Microprocessors and other integrated circuits facilitate the construction of special digital systems for practical image analysis. Various aspects of Digital Image Analysis systems (DIA-Systems) will be discussed in this paper. After a general discussion, a particular system will be presented that the authors have developed.

\subsection{TASKS}

Tasks for practical DIA-systems can be classified from different view points. We choose 3 categories into which these tasks fall: inspection, handling, and control.

\section{1) Visual Inspection}

Quality control is an important task in automated production processes. In many operations the human worker performs an "implicit visual inspection" while handling parts (i.e. he just glances at the part in order to check it superficially). In other instances he makes an "explicit inspection" in order to check the quality of parts more scrutinious $1 y$. Boths kinds of inspection 
are open to automation and it is in this area that we expect widespread use of DIA-systems.

\section{2) Part Handling}

Historically seen, interest in DIA-systems stems from the development of robotics technology: while the first generation of industrial robots was "blind", it was soon felt that a sensor-equipped generation was needed. Obvious tasks for DIA-systems are in the control of industrial robots in handling workpieces. This handling occurs during part transfer, loading and emptying of machines, assembly or sorting. Despite many efforts to introduce industrial robots in a wide range, progress has been slow so far in Europe. This is due to a number of reasons that are outside the scope of this paper. However, this implies that the application of DIA-systems in this area will be even slower.

\section{3) Control of Tools, Machines, and Processes}

This category of applications is of somewhat younger origin, yet there is a high potential of applications to be expected. Here, emphas is is on "inprocess"-control. Examples are the control of tools (e.g. screwdrivers in assembly tasks), the control of machines (e.g. cutting machines), or the control of processes (e.g. Welding). This category may be the most challenging, but with progress in efficiency of DIA-systems a wide range of applications can be expected.

Let us briefly resume these 3 categories with the aid of a few examples (cf. Fig. 1.1 - 1). In row A we list examples for inspection tasks such as: check the presence of parts or presence and completeness of labels on bottles (A1); check the shape of the screw or the completeness of a part coming out of a press (A2); check the surface of running metal sheets or check liquids for particles (A3).

Row $B$ shows examples for control tasks: determine the identity of parts on a conveyor belt including their position-class, location ( $x-y$-coordinates) and orientation (rotation $\phi$ in the image plane) (B1); determine the position of goal sites for assembly tasks (B2); determine the position of the weld head along a seam (B3); in this last example we may also observe the weld pool in order to control welding process parameters. For a systematic view of tasks see /ROSEN $179 /$. 

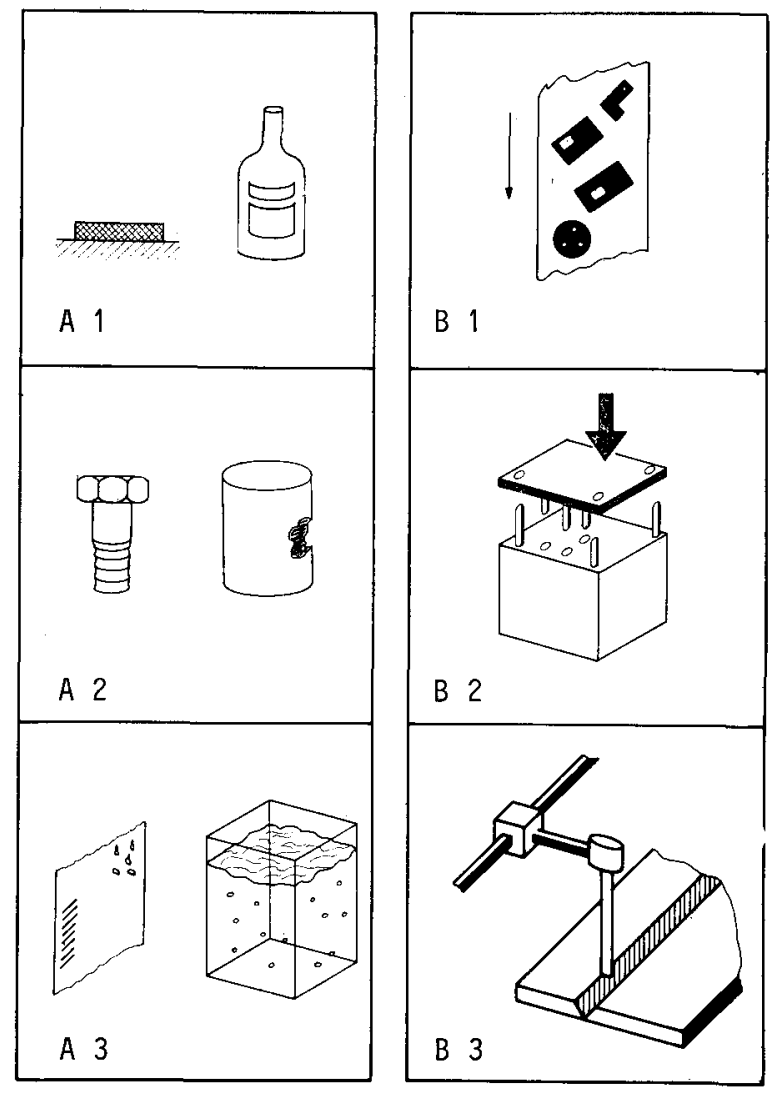

Fig. 1.1. - 1: Examples for Industrial Applications

(see text for explanation)

\subsection{CONSTRAINTS}

A number of constraints determine how effectively a workstation can be automated. In particular one must consider (cf. Fig. 1.2. - 1):

- geometry of the set-up

- degree of order of workpieces

- illumination

- imaging system

- architecture of the DIA-system 
- output processing

- transport \& handling of objects.

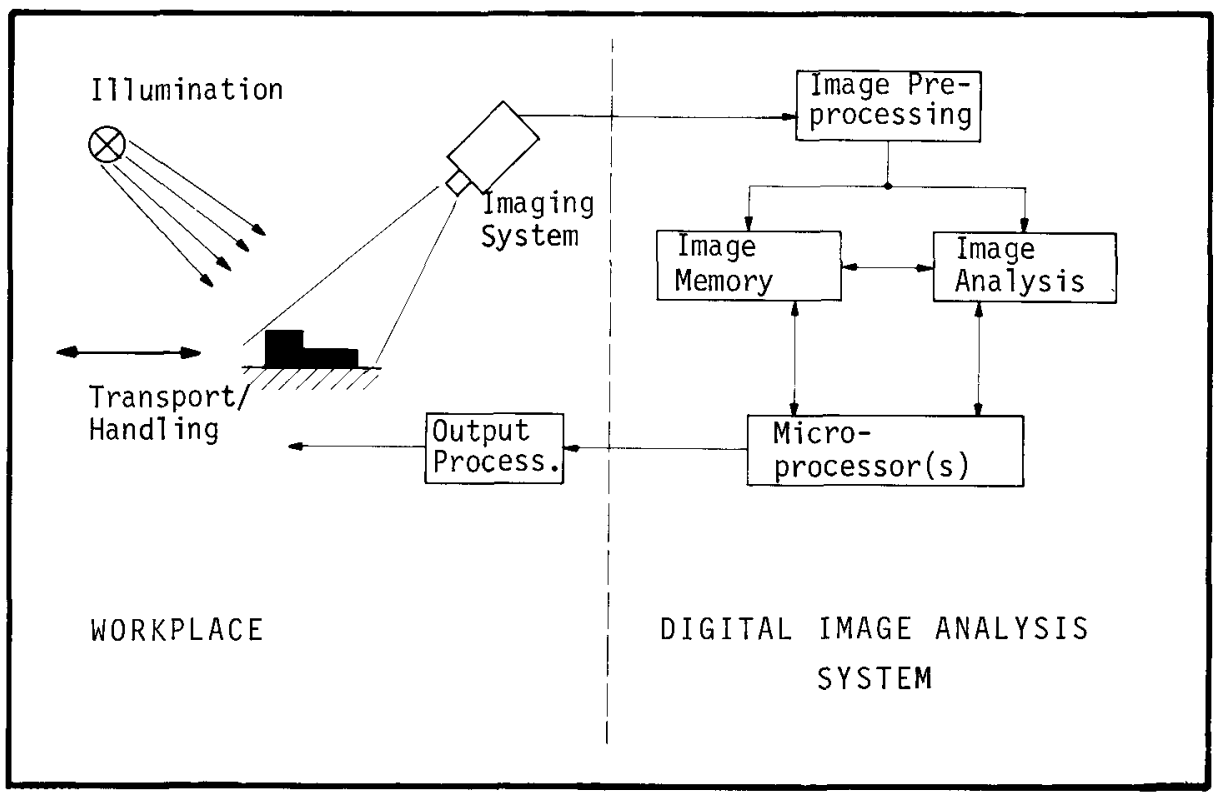

Fig. 1.2. - 1: Components of a workplace

A11 these components are related with each other and must be considered as a whole in order to obtain cost-effective solutions. For example, if the illumination is chosen poorly then one will need a much more powerful DIA-system in order to cope with the inferior quality of images that must be processed.

The geometry of the set-up is determined mainly by the angle and the distance between camera, workpiece and illumination. This geometry can either be fixed or variable. In the latter case the camera is mounted directly onto the manipulator arm and is moved together with the arm. Then both, distance and angle from camera to the workpiece will change permanently and must be considered accordingly. Since that may require a lot of computations (or table look-ups) it is advisible to use fixed geometry whenever possible. If there is a working plane (e. $\underline{\text { g. }}$ conveyor belt) and if the optical axis coincides with the normal of this plane there will be fewer aspects of the objects. It is there- 
fore recommendable to mount the camera directly above the working plane at a fixed distance.

The degree of order of the workpieces is an important factor and a lot of attention must be paid to this part of the problem: the higher the degree of order, the easier the task that the DIA-system must solve (cf. Fig. 1.2. - 2).

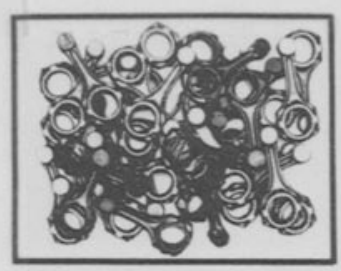

a

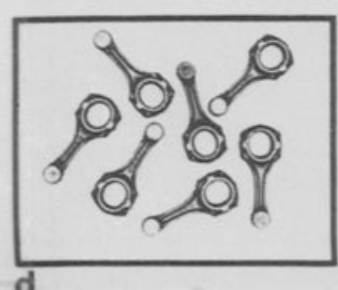

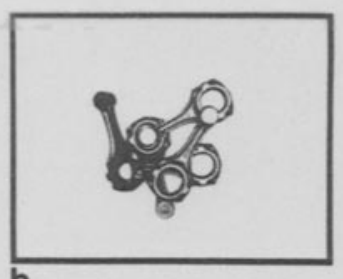

b

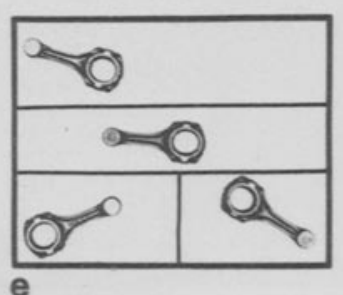

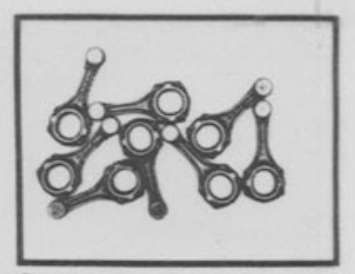

C

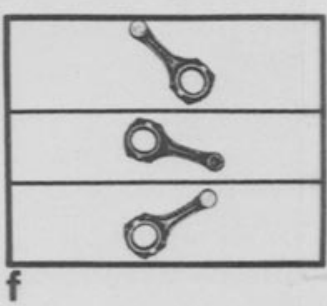

Fig. 1.2. - 2: Complexity of scenes

a - parts in a bin (multiple overlap)

b - few overlapping parts

$c$ - touching parts

d - isolated parts at random

e - isolated parts in semi-order

$f$ - isolated parts in linear order

The complexity of the scene may range from completely loose positions of the parts to completely fixed positions. In the first case parts will overlap and occlude each other (parts in a bin). At the time of writing no DIA-system is known that could analyze such scenes. In the other extreme, when the positions of parts are completely fixed, then there is no need for a DIA-system. Practical tasks for DIA-systems are found in the mid-range. It is obvious that the complexity of the scene determines the required competence of the DIAsystem and thus the costs of the solution.

In general, it can be said, that order is expensive to introduce and maintain Quite sophisticated mechanical periphery is necessary in order to separate 
parts. If one wishes to maintain order it is often necessary to use special magazines. One must therefore find a balance between the costs for mechanical periphery and the costs of the DIA-system.

Illumination can be used in a number of ways to facilitate image analys is. One has the following options (cf. Fig. 1.2. - 3):

- back lighting

- top lighting

- diffuse

- directed

- light sectioning.

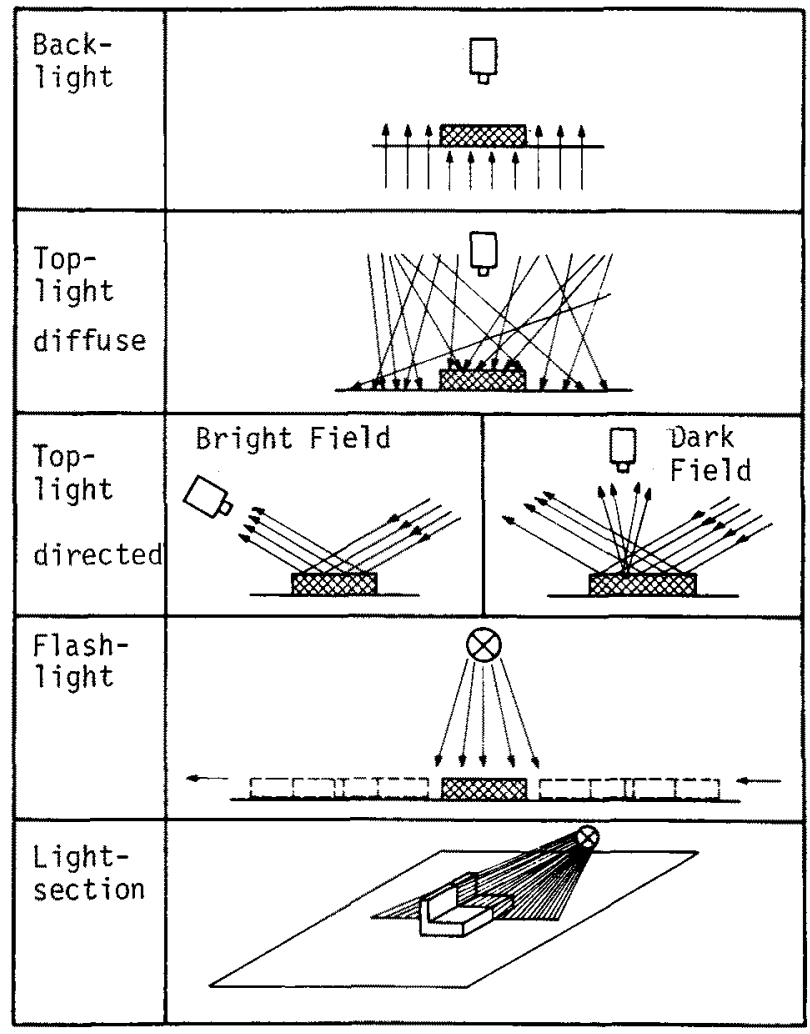

Fig. 1.2. - 3: Illumination techniques

These different illumination techniques can be applied either in steady or in strobe mode. Strobe mode is particularly suitable when motion blur must be suppressed. Back light leads directly to binary images since one only ob- 
serves the silhouettes of parts. Top lighting can be used in different ways: a diffuse light source will in general provide homogenous reflections and thus facilitate the image segmentation process. For inspection tasks (such as measurement of surface smoothness) directed light will often prove to be useful. Here one may observe either directly the reflected light (bright field observation) or the stray light that stems from surface flaws (dark field observation).

Light section techniques provide different informations about the scene than the other illumination techniques. While these deliver surface reflection functions, light sectioning informs about the 3-D structure of a scene. These techniques will not be discussed in this paper. Yet in general, they can be powerful methods for many practical purposes (cf. /HOLLAND et al. '79/).

There are basically two types of imaging systems: 1) CRT-cameras and 2) solid state cameras. In the latter case one can either work with linear cameras or with diode arrays. CRT-cameras have been around for a long time and are readily available. Yet it is assumed that solid state cameras will prevail in the near future.

The architecture of the DIA-system depends on many constraints: required processing speed, image resolution, type of image to be processed (binary vs. gray leve1), complexity of the images, to name the important ones.

A11 practical DIA-systems must work in real-time: quite often this notion is meant as image processing at TV-speed. Here we understand by "real-time image analysis" a process which follows the cycle of the production process. Typical cycle times range from several hundred milliseconds to several seconds. There are of course shorter as well as longer cycle times that can occur in practice. If short cycle times are required, then much of the image processing and analysis must be done by hardware; if long cycle times are allowed one may easily store the image and perform the task by software. The issue of system-architecture will be discussed in greater depth in a later section of this paper. Typically, the output that a DIA-system provides, is some kind of quantified description of the observed scene: what objects are there?; which way do they lie?; what is their exact location?; which way are they rotated?; and so on. Before this information is handed over to the handling system, NCmachine, etc. there is often the need for output processing. Two examples may clarify this point. In the first example, one encounters problems with coordinate spaces when dealing with a sensor-driven industrial robot: DIA-systems 
work in cartesian coordinate spaces $(x, y, z)$ while robots have their own coordinate space which is given by the structure of the robot (polar or cylindrical systems constitute the easy cases; sometimes one has to deal with quite complicated systems). It is therefore necessary to perform coordinate transformations which may be computationally demanding.

A second example may be taken from visual quality contro1. Once the DIAsystem has determined the quality of a part, it must output commands for further treatment of the part. Assume that there are different classes of flaws: those that can be repaired by further processing and those that cannot. That means that the system must decide which step to initialize next.

This brings us to the first/last step in the system: parts must be transported and handled. This issue is related with some of the other components of the system. Parts must be brought to the workplace and also transported from it. While being brought, the parts may be separated and a certain degree of order may be installed. Thus the complexity of the scene is determined in this step. If the parts are moved at constant speed across the sensor field then one might well apply a one-dimensional imaging system (diode line) since the second dimension is provided by the transport system. Transport of parts to the next workplace may also be a crucial step since here input to the next processing system is prepared. If in this step, one is able to maintain the order of pieces then the efforts that have to be made at the next station can be reduced.

Let us briefly summarize the issues of this section. If one wants to automate a workplace it does not suffice to substitute a worker by a handling system/ robot and a sensor-system. Rather, one must consider a 11 components in order to reach an optimal solution. Since most of the components are related with each other, one may reduce the costs of one component by making another component more efficient. This will certainly require a much better cooperation between the mechanical engineer and computer scientist than exists today.

\subsection{EVALUATION OF PRACTICAL SYSTEMS}

From the previous section it has become clear that the performance of a DIAsystem cannot be evaluated per se. Rather, it has to be seen in the context of the complete system. Though one cannot specify a-priori what a good DIAsystem should look like, one can at least list the features that determine the quality of a DIA-system. 
$\underline{\operatorname{COST}}$

Here one must differentiate between costs for the installation and costs for operation and maintenance. The costs for the DIA-system must be related to the complete system whose costs quite often range from $100.000 \$$ to $300.000 \$$. It would therefore seem appropriate to allow $10.000-35.000 \$$ for the costs of the DIA-system. The costs of operation are rather low and can practically be ignored.

\section{RELIABILITY}

This is an important feature since malfunction of the DIA-system could lead to bad damages in the workplace. It is therefore necessary to build failsafe systems that monitor their own out-put. As an example: prior to datatransfer of object coordinates from a DIA-system to a robot controller it must be checked that the coordinates are within allowed ranges.

\section{PROCESSING SPEED}

The required speed is mostly determined by the cycle of the production process. Typical cycle times range from $100 \mathrm{~ms}$ to up to $10 \mathrm{~s}$ although exceptions in both directions occur.

Thus DIA-systems should be able to operate at processing speeds between a few hundred milliseconds and a few seconds. If one wants to process TV images online, $\underline{i}$.e. without storing them, then one must operate in the $20 \mathrm{~ms}$ range.

\section{FLEXIBILITY}

Flexibility is required whenever there are frequent changes of workpieces that must be inspected, recognized or located. This is typical in inspection tasks or robot handling. In these cases it is imperative that the DIA-system can be easily adapted to the new task. This requirement is strongly related with the operability of the system.

\section{OPERABILITY}

Since the DIA-system must be operated at the worksite it should not require familiarity with programing languages in order to operate the system.

Rather, the operator ought to be supported through dialogue-systems, menuing techniques, and problem oriented programming languages that are easy to use. 


\section{MAINTAINABILITY}

Ease of maintainability is a requirement that applies to all kind of equipment and is not a special feature of DIA-systems. Since some of these systems can be rather complex, it may be advisible to install diagnosis routines that help find the source of trouble fast. Modular hardware design for easy replacements is state-of-the-art.

\section{ACCURACY}

The required accuracy that the DIA-system must obtain may vary considerably. Thus one can only specify a typical range: from our experience it can be stated that an accuracy in coordinate measurement around $1 \%$ af the visual field and angle resolution around $1^{\circ}$ are quite typical.

\section{COMPETENCE}

There is a wide variety of tasks that determine what a DIA-system must be capable of. This begins with simple measurements (width, length, area, ...) and stretches out to complex scene analysis tasks. A very crucial point is the following: whatever analysis the system performs, it must be absolutely reliable. There is no use to implement algorithms that "work most the time" since these will not be suitable for practical applications in the industry. 


\section{SELECTED METHODS OF IMAGE AND SCENE ANALYSIS FOR PRACTICAL SYSTEMS}

It is outside the scope of this paper to give a detailed survey on image processing and scene analysis. Rather, a few methods will be selected that seem appropriate for implementation in practical DIA systems. Emphas is is here on practicability, i.e. methods that are feasible for industrial applications in an economic way, now.

Let us briefly define the terms 'Image Processing' and 'Scene Analysis'. In Image Processing one transforms an image into some other image; in Scene Analys is one starts with an image and ends with a description of the underlying scene. Figure 2.-1 illustrates this process: we begin with a graylevel image; the task is to decompose this image into meaningful elements from which a description can be built. In the computer an image is represented as a discrete picture function. In the three-dimensional plot of Fig. 2.-1 we see how the objects in the gray-level image correspond to certain structures in the 3-D plot. By going through a number of processing steps, higher and higher levels of abstraction are obtained until one has eventually reached the desired description.

It is clear that the nature of the description depends on the task the DIAsystem must fulfill. In industrial applications such descriptions could be: number and type of objects in a scene, their positional class, position parameters - in fact all the information that a robot needs in order to grasp objects; statements about the completeness of an object or the quality of a surface (for visual inspection); and so on.

A digital image is a matrix of $\mathrm{N} \times \mathrm{N}$ pixels whose values correspond to gray tones. While the human observer sees lines and regions with meanings rather than pixel matrices, the machine initially "perceives" $\mathrm{N} \times \mathrm{N}$ pixels with no apparent interrelations. It is the task of the processing step to group together spatially neighboured pixels into ensembles that "belong together". This first step is called 'segmentation'. It partitions the image into regions.

There are two basic approaches to segmentation: either by outlining contours or by specifying all pixels inside a region. In the first approach one makes use of differences between the gray values of neighbouring regions; in the second approach one utilizes gray value similarities between pixels within 


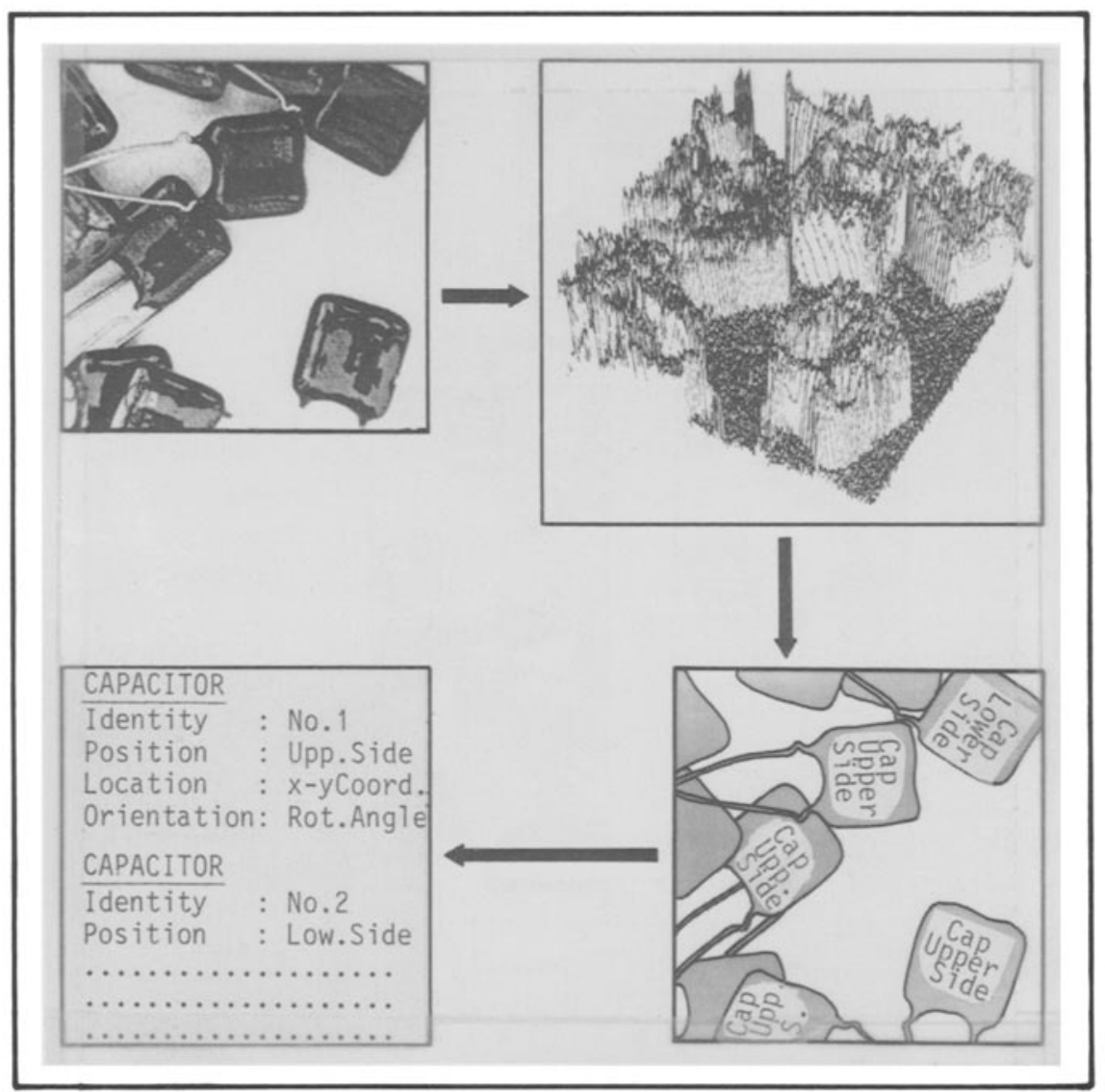

Fig. 2.-1.: Scene Analysis: From Gray-Level Images To Descriptions (the 3-D plot is inverted, i.e. black pixels are high and vice versa)

a region. Sometimes combinations of these two approaches are used - but that will not be considered here (for examples cf. /MILGRAM $177 \mathrm{~A}, \mathrm{~B} /$ or /SCHARF $77 /$ /). Despite the differences between the two approaches both are based on four discrete steps with the same underlying idea: selection/ detection of appropriate points, postprocessing, connectivity analysis, and representation of the resulting structures (cf. Figure 2.-2). 


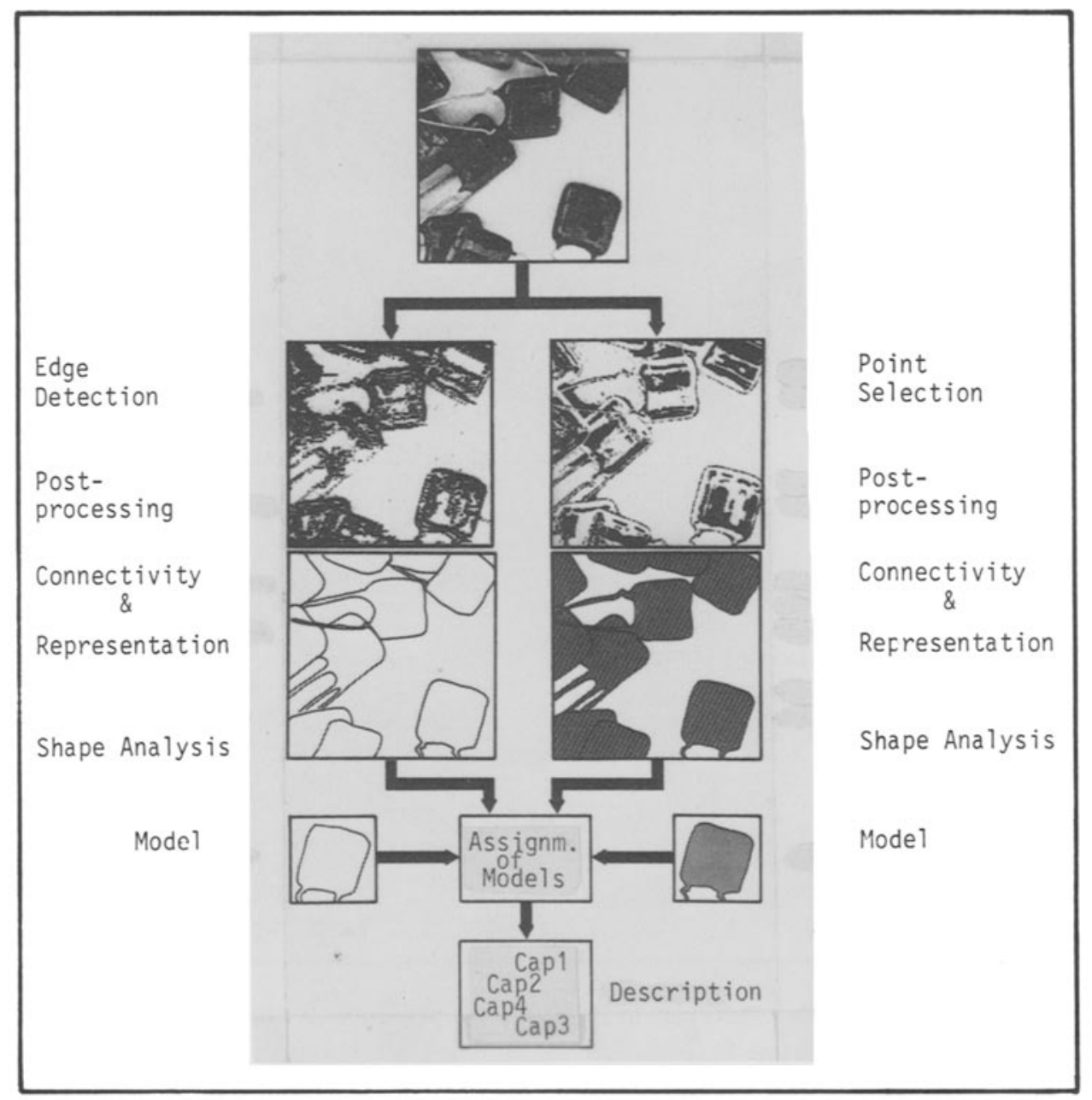

Fig. 2.-2.: Processing Steps During Scene Analys is

With this in mind we can now specify the steps that transform an image into a description:

1) Pixel Selection: in the contour approach this step is the application of edge detection techniques; in the region approach this step can be determined as feature value determination;

2) Postprocessing: Pixel Selection usually leads to noisy results that must be cleaned before further processing is feasible; in the contour approach this could be thinning and gap filling; in the region approach 
this could be the removal of isolated points;

3) Connectivity Analysis: this is an important (and as will turn out:also cruciall step; here one must determine which of the selected pixels "belong together"; in the contour approach this step is performed by line finding; in the region approach this step is usually done by label propagation (or 'component labeling');

4) Representation of Connected Components: once connected components are determined, they must be represented by appropriate means; in the contour approach this can be done by using straight line and curve segments; in the region approach this is somewhat more difficult. Here techniques such as representation by overlapping convex sets can be brought to bear;

5) Shape Recognition: each of the (sub-) structures that are a result of the segmentation process must now be recognized. For this the shape of the element can be used. Shape analysis may be used to assign symbols to each of the elements. These symbols may be compared to some kind of vocabulary with which to describe the visual appearance of objects;

6) Assignment of Models: by using the afore mentioned vocabulary we can express through appropriate data structures what objects 'do look like. With the aid of this a-priori information we can now compare extracted elements and structures with stored models and assign matching models to groups of image elements. The record of these assignments is then the desired description (this implies that the models contain all the information that one seeks).

This scheme is a rather generalized one which completely ignores control structures among the various steps. There are many ways how to introduce feedback loops between all processing steps; quite seldom will the analysis proceed as straightforwardly as has been presented here. Ideally, all steps should be provided with as much a-priori as well as a-posteriori information as possible. Yet, it is outside the scope of this paper to discuss any issues of control structures.

In the following we will briefly discuss various techniques for segmentation, shape analysis, and assignment of models. We will emphasize here which techniques are ready for real-time applications and which are not.

\subsection{SEGMENTATION}

As has already been mentioned the goal of segmentation is the partitioning of an image into meaningful entities. In Scene Analysis there are of course many semantic levels. It must therefore be determined at which level the image is to be described. Low levels describe the image in terms of local features such as boundary elements or homogenous reflections and provide only general information. Intermediate levels describe the image in terms of 
regions and lines and provide knowledge about object surfaces. Higher levels describe the image via object parts or objects and provide specific knowledge. In workpiece recognition it seems appropriate to segment the image into regions that correspond to visible surfaces. Due to variations in illumination, reflectance, and surface orientation it will of ten be impossible to establish exactly that correspondance. The process of segmentation will therefore result in an incomplete partitioning.

There are two basic approaches to segmentation: via contours or via regions. Both approaches will be discussed in this section. It has been pointed out that both approaches are based on 4 steps. Of these, 2 steps are most important: pixel selection and connectivity analysis. It is important to understand that these two processes are principally different: the process of pixel selection is based upon properties of the intensity array, connectivity analysis is based on spatial continuity.

\subsubsection{SEGMENTATION VIA CONTOURS}

The basic idea of this approach is to delineate the regions into which the image is to be segmented. This implies that neighbouring regions must differ sufficiently in gray level from each other. We exclude here the problem of texture discrimination. The state-of-the-art is still a long way from segmentation through texture discrimination. We therefore assume for the remainder of this section that all regions in the image are homogenous to some degree or at least not heavily textured. This is often true in industrial environments. If not, other techniques must be brought to bear for the segmentation process.

Boundaries of homogenous regions are in general edges, $\underline{i}$.e. step-like structures in the intensity array. As can be seen from Figure 2.1.1.-1 real edges are not clear cut steps but vary considerably in shape. The first step in segmentation is the process of edge detection. Here one determines which pixels are possible edge points and which are not. 


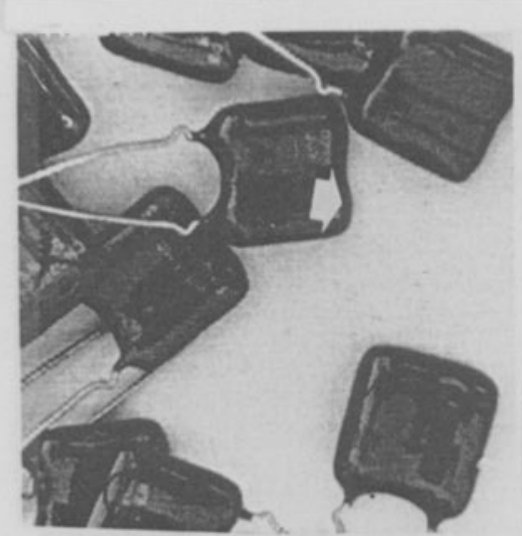

A

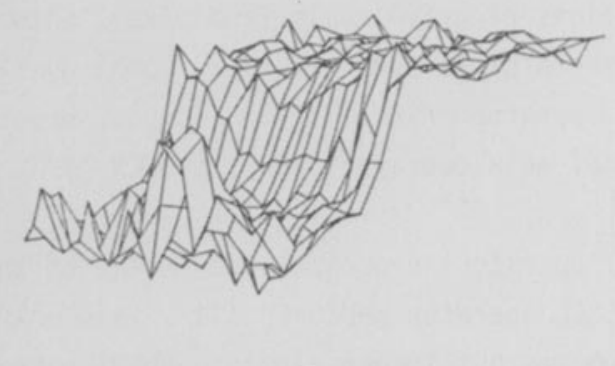

B

Fig. 2.1.1.-1.: The Three-dimensional Structure of A Real Edge

A - Gray Level Image (see arrow!)

B - 3-D Plot

Basically, the following kind of information can be extracted during edge detection:

- local orientation ef edge elements;

- strength of edge elements, i.e. the contrast in brightness between neighboured regions;

- width of edge elements (since edges are not ideal steps they can vary in width considerably);

- placement of edge representant; since edges may be more than 1 pixel wide, it is necessary to determine where to place the edge point;

- polarity of the edge element (which of the regions is the brighter one?);

- gray value of the edge element;

- gray value of the neighbouring regions.

There is a huge number of operators available for edge detection. Since there exist excellent surveys /DAVIS '75/, /LEVIALDI ' 80 / we will not give any details. Typically, most operators fall in one of the following three classes: local, regional or global operators. 
Local operators process a relatively small part of the image at one time. Typical window sizes are $2 \times 2,3 \times 3$ pixels; in general window sizes don " $t$ exceed $5 \times 5$ pixels. The performed operations are linear or non-linear combinations of gray-levels from pixels within the window. A few examples are: Roberts cross /ROBERTS '65/, Sobel operator/TENENBAUM et a 1. '69/, Prewitt operator /PREWITT 170/, compass gradient operator /ROBINSON 177/, or orthogonal mask operators /FREI \& CHEN $177 /$.

Regional operators process larger areas of the image. A typical example is the HUECKEL operator /HUECKEL 171. This operator does not detect point-1 ike edge elements but longer elements that cross a (circular) region. Simplified modifications of this operator have been developed by /MERO \& VASSY '75/, /0'GORMAN '78/, /BUROW \& WAHL '79/ and a number of other authors.

Global operators are those that process the complete image. Typical examples are filtering approaches such as high pass filtering/ROSENFELD \& KAK $176 /$ or Wiener filtering /FRIES \& MODESTINO $177 /$.

A

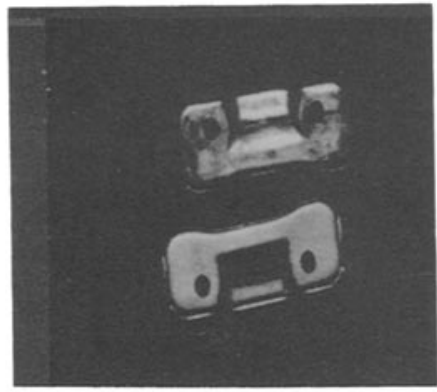

D

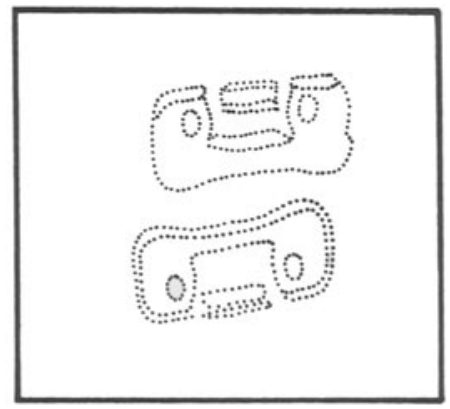

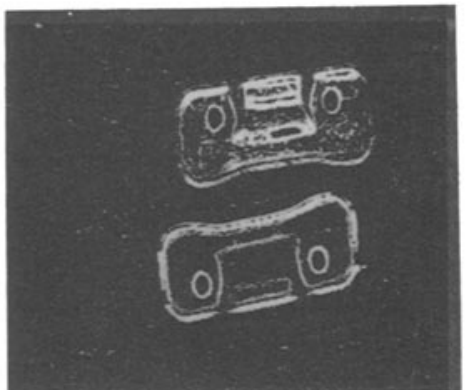

B

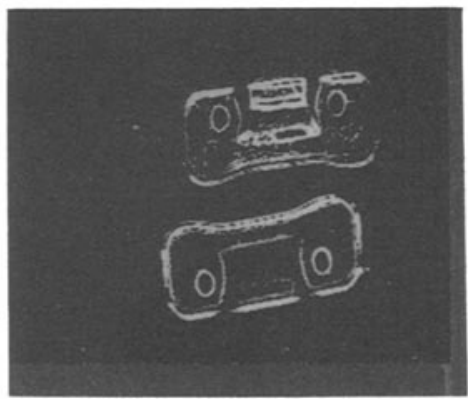

Fig. 2.1.1.-2.: Local Edge Detection

A - Gray Level Image

$B$ - Gradient Image (Sobel Operator)

C - Thresholded Image (B), THRSH $=40$

D - A Reminder: We still have a matrix of points, and no 1 ines! 
Figure 2.1.1.-2 shows an example for a local edge detector - the Sobel operator. There are at least 4 problems that one has to cope with at this stage:

- noise (even background pixels yield some edge value);

- smear (parts of the contours are rather thick);

- fragmentation (some parts of the contours are missing);

- misplacement.

These problems make it necessary to apply some postprocessing which provides line thinning, gap filling, as well as noise suppression. The most obvious technique is of course thresholding, i.e. the suppression of all points whose edge value is below a given threshold. Clearly thresholding removes noise to a certain degree, yet it is neither suited for gap filling nor line thinning. Better postprocessing techniques make use of local context and yield far better results. The most commonly used techniques are:

- non-maximum suppression

- local connectivity analysis

- relaxation.

A11 three techniques are based upon much the same idea: if a pixel shares similar edge directions with its neighbouring pixels then it is likely to be an edge point and will be enhanced; otherwise it will be suppressed. In non-maximum suppression the considered neighbourhood consists of the left and right neighbour across the edge. If either of these has a higher edge value, the considered pixel is suppressed. For discussions of these techniques see /ROSENFELD \& THURSTON 171/, RISEMAN \& ARBIB $177 /$ or /PRAGER '80/. In local connectivity analysis one looks at a $3 \times 3$ window around the actual pixel. Here, continuation along the edge point is checked: if there are preceeding and succeeding pixels with similar edge directions, then the actual pixel is considered an edge point. In the approach of /ROBINSON 177/, /ROBINSON \& REIS 177/ similar edge values are also required; if these exceed an adaptive threshold value and edge continuity is given, then the pixel is entered into a binary edge map.

The techniques of non-maximum suppression and connectivity analysis work in one single step and throw away all the information that is contained in those pixels that are suppressed. The third technique, relaxation, makes use of these informations as well and constitutes a generalization of the other techniques. The basic idea of relaxation is to set up a 'cooperation and 
competition' between neighbouring pixels with respect to their local interpretation. Let us rather briefly clarify this point: contrary to non-maximum suppression the edge values of weaker elements are not thrown away but are 'collected' by the stronger elements. In other words, strong edge points that are consistent within their local context are enhanced further and further while weak and/or inconsistent elements are gradually diminished in importance. Relaxation schemes work basically in parallel and iteratively; strength or weakness of elements is expressed by probabilities of labels and the process of 'value collection' is performed by changing these probabilities in dependence of local context. We are not going to discuss these techniques any further but refer the reader to /ROSENFELD, HUMMEL \& ZUCKER ${ }^{1} 76 /$, /ZUCKER, HUMMEL \& ROSENFELD ' 77/, /ROSENFELD ' $77 /$, /RISEMAN \& HANSON ' $78 /$, /RISEMAN \& ARBIB ' $77 /$, /PRAGER ' $80 /$ or/PERKINS ' $80 \%$

As a result of these postprocessing techniques one obtains edge images with thin lines and filled gaps. Yet, while the human eye sees clear lines, in the computer we still have a matrix of single edge points that must be linked in order to form lines. The process of line finding is certainly one of the most difficult steps in image analysis. In comparison with the huge variety of edge detection techniques there are only few line finding techniques available today. We want to classify these into 3 basic approaches (although other classification schemes might well be more suitable):

- local methods

- global methods

- iterative methods.

Typical local methods link edge points by starting at an appropriate point and looking for good continuations in the next line /ROSENFELD \& KAK $176 /$, /KORN '78/. If some criterion doesn't hold any longer that line is abandoned and another line is started. Yet another local linking scheme consists in binding edge points pairwise into 'streaks' /SLANSKY '78/, /NEVATIA \& BABU 179/. Among the global approaches we see two different types of methods: 1.) tree search techniques that evaluate a 'goodness' function while they go along and

2.) transform or template matching techniques that determine what prevails in the image.

Among the tree search approaches we find techniques such as heuristic search /MARTELLI '72/, dynamic programming /MONTANARI $171 /$, /EHRICH $177 /$, minimum 
cost tree search /ASHKAR \& MODESTINO $178 /$, and locus search /YACHIDA, IKEDA, \& TSUJI 179/. Among the important transform techniques for line finding there is the Hough transform which transforms an edge image into a 2-dimensional parameter space where collinear points cluster /IANNINO \& SHAPIRO 178/. It can be shown that this transform corresponds to template matching /STOCKMAN \& AGRAWALA '76/. A11 these techniques on ly make use of information that is available in the image.

The last (iterative) approach makes use of a-priori information as we11: here one first extracts major contours that indicate what object is present; finer contours are then found by model-driven programs /SHIRAI '78/.

Let us evaluate how well 1 ine finding approaches work. Very generally speaking, it can be said that line finding methods deliver results that are far from what the human observer perceives. Figure 2.1.1.-3 shows a typical example of the problems that one encounters in line finding: lines are missing, lines are too short, lines are too long, lines have the wrong direction, lines are fragmented into small pieces, there are duplicate lines.
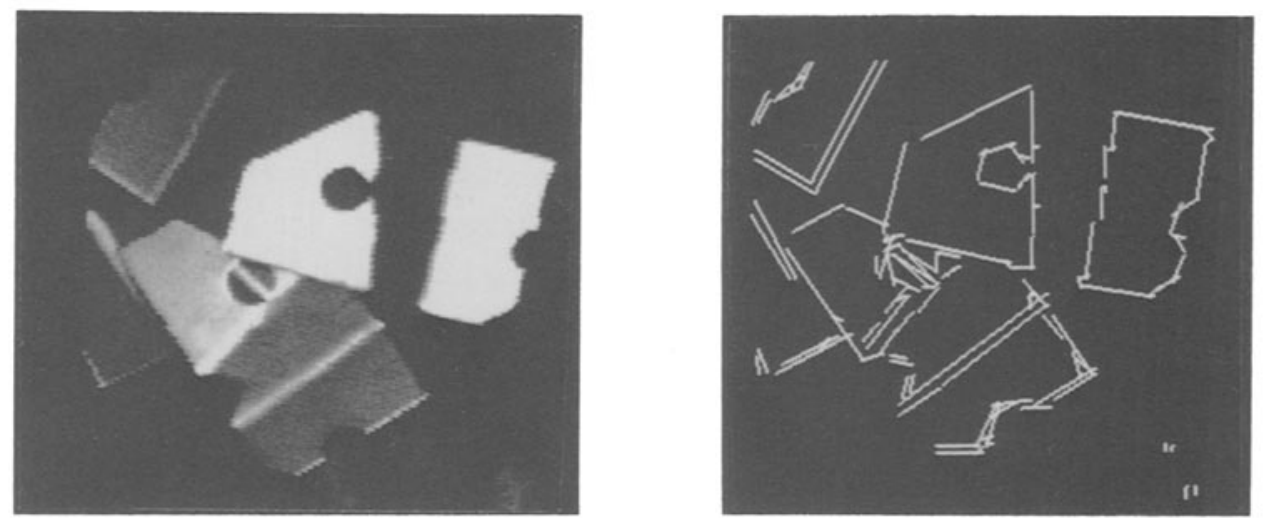

Fig. 2.1.1.-3: Line Finding

A - Gray Level Image

B - Lines Extracted By Line Finder From /KORN '78/ 
The final step in contour finding is yet another postprocessing with the aim of cleaning up resulting lines. Here, short line elements are removed or linked into longer segments, if possible. Thus one finally obtains a list of contour 1 ines that are the result of the segmentation process.

How well suited is this segmentation approach for real-time processing and for practical applications in the industry? Recall from the introduction that there are three important issues which are related with the practicability of DIA-systems. These are: real-time processing capability, cost effectiveness, and reliability.

Let us briefly discuss real-time processing via contour segmentation. Given the processing speed of today's micro-electronics one must rely upon local computations where relatively few data have to be handled. It would therefore seem most appropriate to develop dedicated hardware for local edge detectors. This is indeed happening in a number of various efforts. Examples are: the Sobel chip in CCD technology /NUDD et al. 177/; a multifunction chip which includes a $5 \times 5$ programmable transform, a $3 \times 3$ Laplacian operator, a $7 \times 7$ mask programmable kernel, a $5 \times 5$ cross shaped median and a bipolar convolutional filter for $26 \times 26$ pixels /NUDD et al. 179/; discrete circuitry for the on-1 ine computation of the gradient /ZURCHER '79/; or a development of JPL which performs computation of gradient, construction of an 'Edge Map' and postprocessing within an 8-pixel-neighbourhood /ESKENAZI \& WILF 179/; one even investigates VLSI technologies for image processing algorithms /EVERSOLE et al. 179/. All these processors are specified to work with TV speed, i.e. they are capable of real-time processing. There are also developments that support real-time postprocessing: connectivity analys is is performed by a 'real-time edge processing unit' from Northrop /ROBINSON \& REIS 177/; the development of cellular structures would support the application of relaxation algorithms /WILLETT et al. 179/; the development of a local gradient direction histogrammer /BIRK, KELLEY et a1. '80/ prepares the extraction of straight lines. The really hard problem is the line finding stage. Since this is a search process backtracking may be necessary. Today, we are not aware of hardware projects that would support search processes. A step in the right direction seems to be the development of'SPARC'/ALLEN \& JUETTEN 178/. Although postprocessing facilitates the line finding process it is only possible to extract major contours in a reliable way. In general it can be said that contour segmentation requires high computational time (or much specialized hardware), large memories, and a fine tuning of 
many parameters and thresholds. Despite the developments for real-time edge detection it does not seem feasible to base practical DIA-systems upon contour segmentation. With few exceptions it will take some time before these techniques can be applied for industrial purposes.

\subsubsection{SEGMENTATION VIA REGIONS}

The alternative approach to segmentation is to specify which pixels belong to a certain region. Again, segmentation is based upon 4 discrete steps,viz.: point selection, postprocessing, connectivity analysis, and representation. We will advocate the use of thresholding techniques and completely ignore methods of region growing since these do not seem appropriate for industrial applications.

When using thresholding one should state the assumptions that one makes about the nature of the images:

- at least some of the object surfaces must reflect homogenously;

- there should be no heavy textures in the image;

- illumination should be homogenous.

In industrial applications these assumptions are often true. Most workpieces (metal, plastic, ceramic, and so on) tend to have smooth surfaces. Most often parts are presented to the DIA-system during the process of manufacturing; they are therefore 'brandnew' and rust or other soiling are scarce. Since the illumination can be adapted to the task one can make sure that it will be homogenous. Even if not, one could still apply local adaption techniques which can be applied as real-time preprocessing systems /WEDLICH '77/.

Let us briefly discuss the various steps of region segmentation. Fig.2.1.2.-1 shows a simple gray value image that fulfills our basic assumptions. The 3-D-plot of Fig. 2.1.2.-1.C reveals that there are basically two different populations of gray levels, viz. gray and bright ones. These appear in the gray level histogram as one large mode (for gray values) and a small plateau to its right (for the bright pixels). Of course gray pixels correspond to the background, bright pixels belong to object surfaces. If we now select a threshold such that it lies between the right flank of the mode and the beginning of the plateau we obtain a binary image as in Fig. 2.1.2.-1.D (above-threshold pixels are colored black as 'figure', 


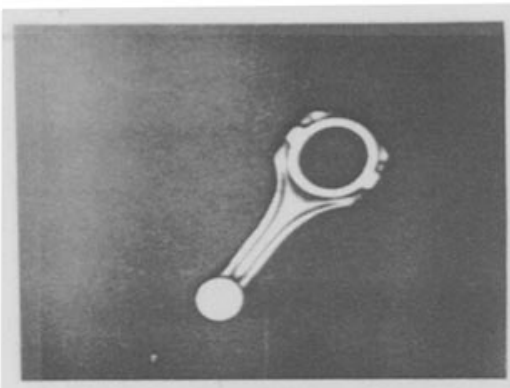

A

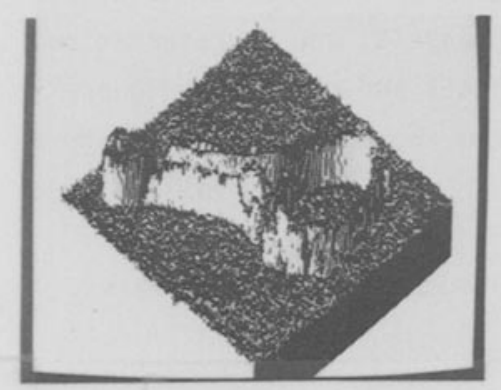

C

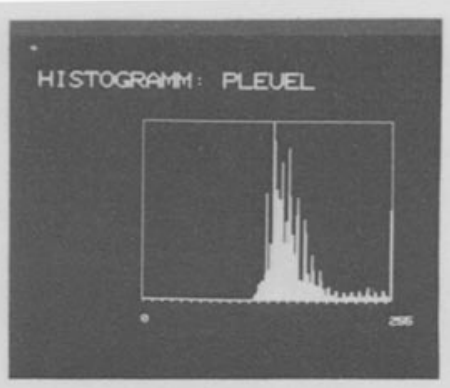

B

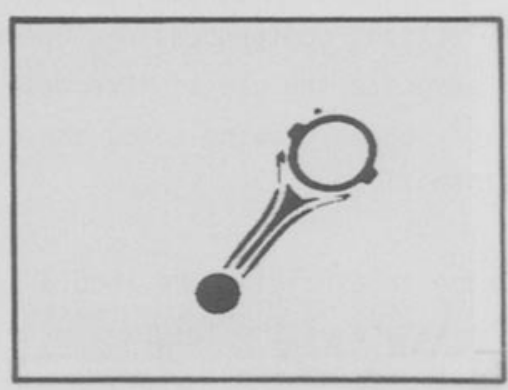

D

Fig. 2.1.2.-1: Image Segmentation Trough Thresholding

A - gray level image

B - gray level histogram

C - 3-D plot

D - binary image

below-threshold pixels are colored white). Obviously much depends on the choice of the right value for the treshold.

The selection of thresholds has found quite some interest in the literature and there are various approaches. One can work with

- fixed thresholds

- adaptive thresholds

- image dependent thresholds

- result dependent thresholds.

Fixed thresholds are determined interactively by a human operator; usually the same threshold value is used all over the image. This works well when one has complete control over the illumination and observation conditions. 
Adaptive thresholds are determined from local information (either by computing an average gray level /TOKUMITSU et a1. 178/ or a histogram in a local neighbourhood /NAKAGAWA \& ROSENFELD '78/). Adaptive thresholds can cope with local changes in the 11 lumination. Image dependent thresholds are selected

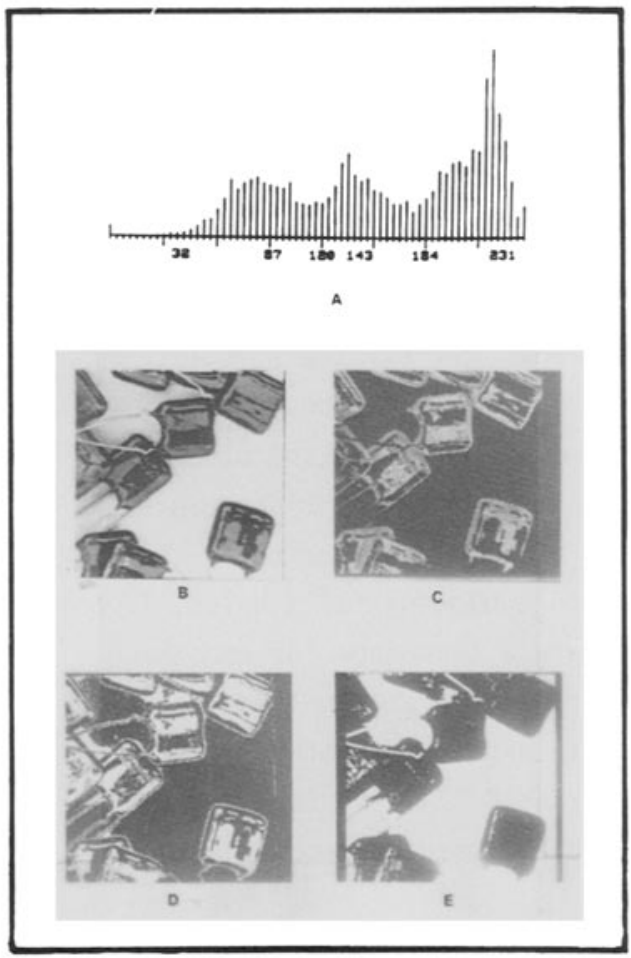

Fig. 2.1.2.-2: Threshold

Selection From Histogram Analys is

A - gray level histogram

$B$ - gray level image $(0 \quad-255)$

C - gray level slice $(32-120)$

D - gray level slice $(121$ - 183)

E - gray level slice $(184-255)$

from global histograms. Fig. 2.1.2.-2 shows the basic idea of this approach: often, modes in the gray level histogram correspond to pixels that belong to the same region in the image. Thus by selecting threshold values at valleys that separate modes in the histogram one is able to extract exactly those regions. The classic example for this approach is /OHLANDER et a1. ${ }^{178 /}$ (a) though they used color images). Instead of using gray values one may also analyze edge value histograms. Here one determines the right shoulder of the histogram where the second derivative is maximum /BAIRD 177/. Yet another and more powerful technique is the analys is of 2-dimensional histograms; here gray value is plotted against edge value /PANDA \& ROSENFELD 178/, /MILGRAM \& HERMAN 179/. As a result one obtains varying thresholds that depend on the location of pixels: pixels within a region are thresholded differently than those on a boundary. This technique is reported to lead to good results. Finally, result dependent techniques start with some threshold 
that was randomly selected and use the result to derive a new threshold /RIDLER \& CALVARD 178/. Even after selection of a good threshold there can still be noise in the resulting image. Typically this noise occurs as:

- small biobs or isolated points

- small holes within blobs

- fringed border lines of blobs.

We therefore need postprocessing techniques that can perform suppression of small blobs, hole filling, as well as border smoothing. There exist indeed operators that are well suited. These have long been known in the 1 iterature as 'shrink' and 'blow' /ROSENFELD \& KAK '76/ and are realized in several systems such as the T.A.S. /KAMIN 174, /NAWRATH 179/ or others /LOFFLER \& JAGER '79/. Shrinking erases all pixels that have a neighbour in the background and thus 'erodes' the blobs; blowing works just the other way and adds another layer of border points thus 'dilating' the blobs. Both techniques can be applied iteratively. It is obvious that shrinking eliminates small blobs and isolated points while blowing fills holes. If both techniques are applied sequentially one obtains a technique for smoothing border lines. Here the order in which these operators are applied plays an important role: 'blow - shrink" tends to close gaps and smoothes border lines; it is therefore called 'Fermeture' /KAMIN '74/. On the other hand, 'shrink - blow' tends to deepen cracks in the borders and removes small noise; it is therefore called 'Ouverture'.

After postprocessing the connectivity analysis must take place. Since at this step all connected components in the binary image are marked with an identifying label, this process is often called 'component labeling'. There are many algorithms that perform component labeling. A few examples are /ROSENFELD \& KAK '76/, /KRUSE '73/, /MORI et al. '78/, /DUFF '76/, /VEILLON 179/, /AGRAWALA \& KULKARNI 177/. Instead of discussing any of these algorithms let us briefly give the basic idea of those algorithms that are based on a top-down, left-to-right image scan (such as the TV scan); cf. Fig. 2.1.2.-3: when we scan the image in this fashion we store linewise the intersections of the scan with connected components (determination of linewise connectivity is obvious); each time we cross a component that was not previously encountered, a new label is assigned to that section; while scanning the next line one checks for overlap of sections in the two lines; if an overlap occurs then the actual section gets assigned the same label as the section 


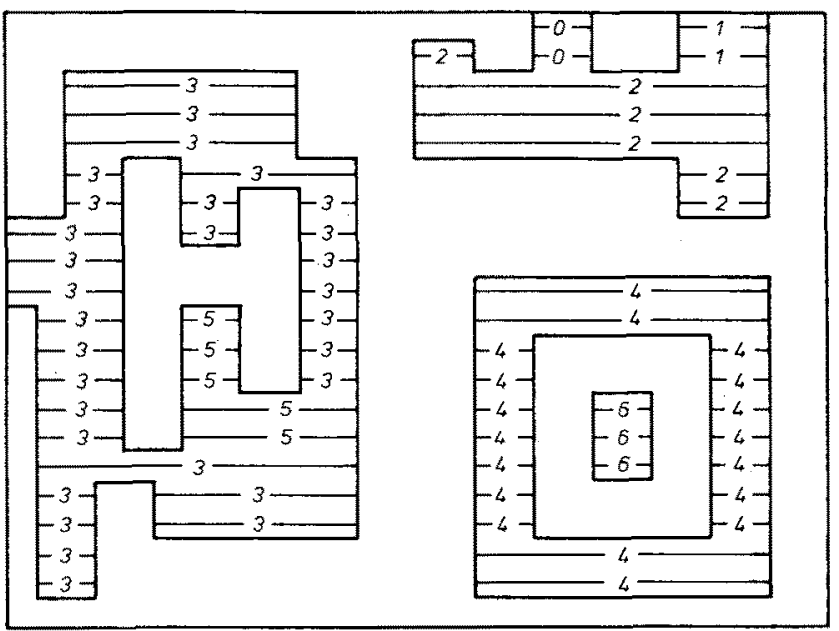

Fig. 2.1.2.-3 : Component Labelina In The Order of Appearance During A TV-scan

from the previous 1 ine. As can bee seen from Fig. 2.1.2.-3 care must be taken when two different labels converge; here one stores this event in an 'equivalence table' which can later be used for relabelina.

After component labeling each blob in the image can now be identified through its set of labels. If the labels are assigned to each pixel of the blob then this constitutes a representation of that blob already (though not a very efficient one!). Another way of representation maybe the decomposition of each blob into regular subfigures such as convex sets, cf, for instance /ZAMPERONI '78/. More decomposition techniques can be found in /PAVLIDIS '77/, /HARALICK \& SHAPIRO '77/, /FENG \& PAVLIDIS '75/, or /PAVLIDIS '72/. A11 these techniques represent blobs explicitly in terms of substructures or primitives. Such techniques are seldom used for industrial applications. Far more often one derives directly some shape feature from the labeled blobs (see the next section) and stores those features together with the labels.

Let us briefly evaluate how well suited the region segmentation approach is for real-time processing. It is clear that thresholding can be easily performed with TV speed; the same holds for operators such as shrink and blow: these are local operations that can be easily performed with high speed (and this has been done a long time). Thus the only critical step could be 
the component labeling but here experience tells us that it can indeed be done with TV speed (cf. chapter 3.). One example for a hardware realization in CCD technology is /WILLETT \& BLUZER '77/. Thus we may conclude that this approach is well suited for industrial applications and real-time processing. Therefore it does not come as a surprise that almost all practical DIAsystems that are available today for industrial purposes are based on the analysis of binary images that are obtained through thresholding.

\subsection{SHAPE, MODELS, AND MATCHING}

After the process of segmentation (and postprocessing) the image is decomposed into a set of discrete structures. An ideal segmentation would yield exactiy the silhouettes of a 11 workpieces that are present in the image; we understand here by 'silhouette' the set of all pixels that the image of a workpiece covers. In reality perfect silhouettes are only obtained when backlighting is used (since this leads directly to binary images). Otherwise one must expect segmentation results that are quite imperfect and where only parts of the silhouettes are extracted. In the contour approach we may obtain only some of the major contours (cf. Fig. 2.1.1.-3); in the thresholding approach silhouettes will fall apart into a set of blobs as in Fig. 2.1.2-1.

In order to analyze the complete image we must therefore:

- assign some meaning to the extracted structures;

- group these structures such that they correspond to the images of workpieces that one is looking for. 
In order to assign some semantic label to the extracted structures one must recognize them. This is done by shape analysis of contours or regions (a) though other information such as contrast, polarity, or gray level could also be used). Approaches for shape analysis for both kinds of structures will be discussed in this section. After recognition of structures one must determine in which way they "belong together". The search for meaningful ensembies cannot be performed efficientiy in a blind way. Rather, it must be guided by models that specify what to look for. Therefore, the system must contain a set of models that describe all aspects of workpieces that are to be recognized; the process of recognition then consists in matching the extracted data with those of the models. Model structures and matching techniques will also be discussed in this section.

Shape analysis is a difficult problem (cf. Fig. 2.2.-1). There is no "Theory of Shape" but there are many - sometimes singular - approaches to shape analysis. Let us look at two principles according to which the manifold of approaches can be cast into some kind of scheme (cf.Fig.2.2.-2):

1) what spatial property is used for the analysis?

2) Is the result of the analysis a number (or a vector) or is it a structure (such as strings, trees, graphs,...)? 


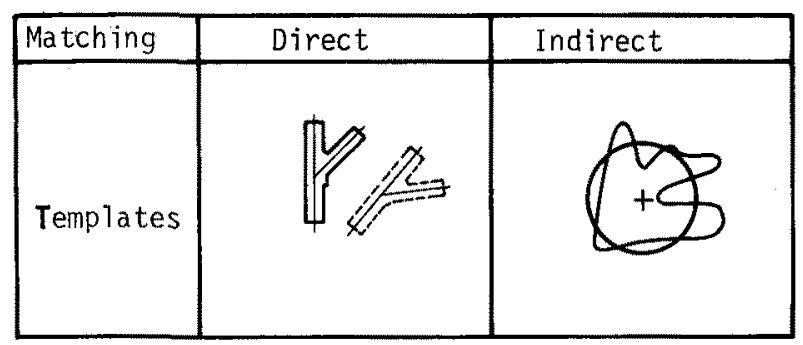

\begin{tabular}{|l|l|l|}
\hline Features & Scalar & Structural \\
\hline contours & \\
\hline Regions & \\
\hline
\end{tabular}

Fig. 2.2.-1: Methods of Shape Analysis

We can either take the complete figure and compare it with some reference (template matching) or we can derive features from contour or regional properties. If we obtain numerical features we talk about 'scalar' methods; if the result of feature extraction leads to structures we call these methods 'structural'. It should be noted that there are no clear boundaries between these categories.

Template matching can be performed either directly by using iconic references or indirectly by applying an artifical template (such as circles around the centroid of the figure). The resulting intersections between the template and the figure deliver features that can be used for classification as well as for the determination of orientation in the image plane. Direct feature extraction can be based on contour or region analysis. In the first case "one walks around the figure", in the second case "one walks 


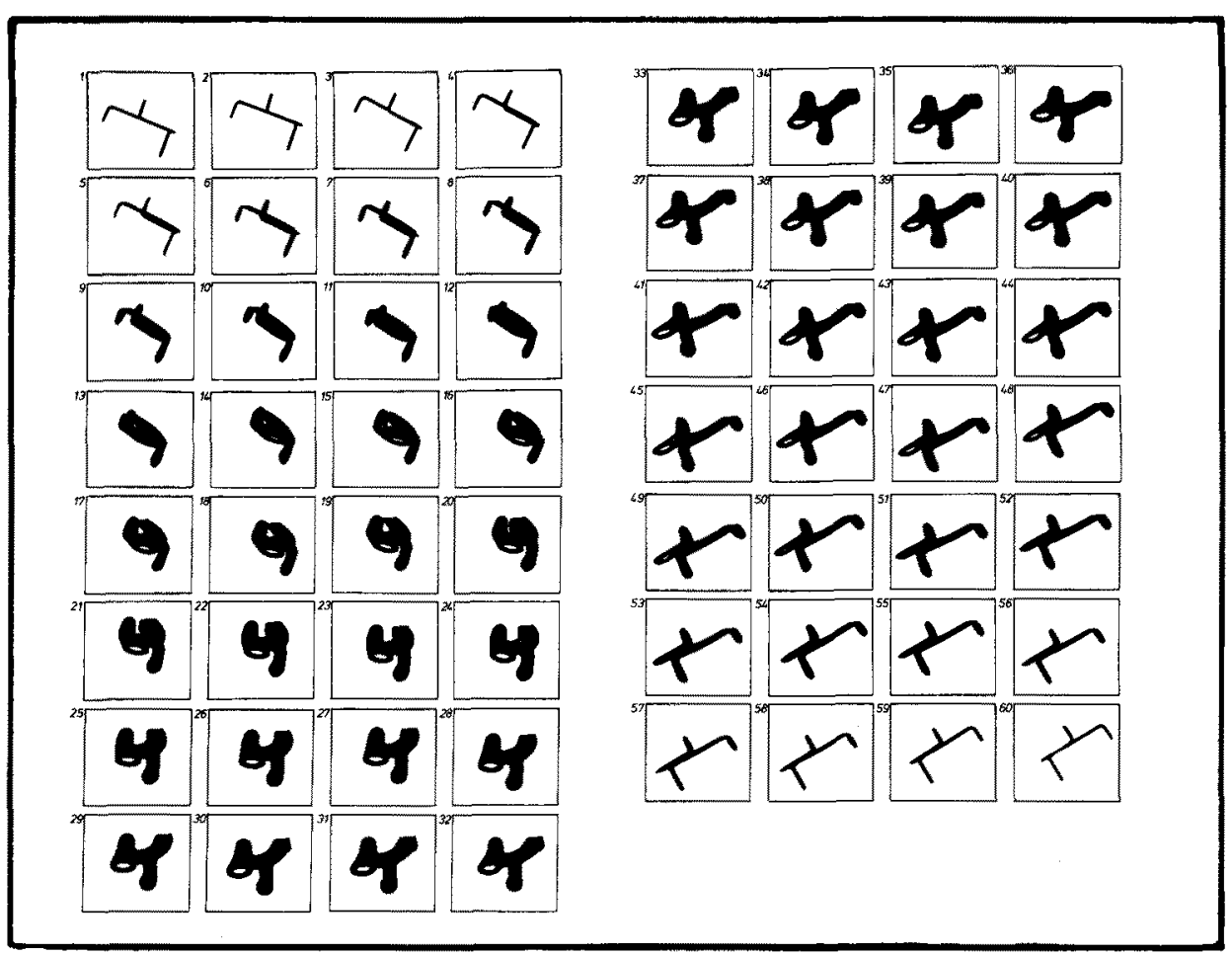

Fig. 2.2.-2: Shapes of Workpiece Silhouttes /FOITH '78/

inside it"/PAVLIDIS ' $78 /$.

Examples for scalar features that can be extracted from contours are: perimeter; minimal, maximal or average curvature; minimal, maximal or average polar distance (= the distance of contour points from the centroid). Scalar features that can be extracted from regions are: area, moments of inertia, number of holes,... If one works with scalar features then usually numerical classifiers are used for the assignment of semantic labels. Typically, nearest neigbour classifiers prevail /DUDA \& HART 173/. These can be modified to improve their performance by adding tests in each feature dimension /FOITH 178/. Another powerful classification technique is the decision tree approach where features are tested sequentially in order to determine an optimal path through a search tree /ROSEN et al. ${ }^{176 /,}$ /GIRALT et al. '79/. 
Examples for structural features that can be extracted from contours are: straight line elements or elements with constant curvature. Structural features that can be extracted from regions are: convex subsets or other regular substructures. Structural features require different analys is methods; here either syntactic or heuristic approaches are used. In the syntactic approach structural elements are considered as primitives of a vocabulary; shape analysis is then performed through parsing. It is outside the scope of this paper to discuss the merits and drawbacks of the syntactic approach. Generally, it can be said that the basic weakness is the sensitivity of this method to disturbed data (although work is going on to make this approach more flexible). Heuristic approaches are far more flexible in this respect, yet they sometimes lack generality.

In practice, all kinds of approaches have been used for industrial applications. Let us give a few examples. /BAIRD ' $76 \mathrm{~A} /$ uses local and global templates to determine the corners of IC chips (see also/BAIRD 178/). Indirect template matching techniques are used by: /BRETSCHI 176/ who uses TV-1ines to intersect object silhouettes or by /HEGINBOTHAM '73/ and /GEISSELMANN '80/ who use circles around the centroid. Examples for scalar methods of contour analysis can be found in: /AGIN 175/, /PAVLIDIS 177/, /DE COULON \& KAMMENOS $77 /$, /DESSIMOZ '78/, /NAKAGAWA \& ROSENFELD $179 /$ or /ARMBRUSTER et al. '79/. One particular advantage of working with contour features is the fact that these can be used when parts are overlapping each other. Here, contour features provide local cues that can be used for recognition of partial views /MCKEE \& AGGARWAL 177/, /PERKINS 177/, /KELLY, BIRK \& WILSON '77/, /DESSIMOZ et al. '79/, /TROPF ' $80 \%$.

Regional features such as area and moments have been used by /BIRK, KELLEY et a $1.76 /$, /BAIRD $76 /$, /HASEGAWA \& MASUDA $77 /$ or /FOITH $178 /$. The syntactic approach, finally, has been applied by /MUNDY \& JOYNSON $177 /$ and /BJORKLUND $177 /$, among others. Structural, regional features seem to have found only little interest - probably because decomposition into regular substructures involves high computational costs. With the support of dedicated hardware most methodes discussed here are well suited for real-time processing and industrial applications.

As can be seen from these examples the boundary between shape analys is and model matching is rather fuzzy. The simplest models that can be constructed for workpieces are just lists containing shape features; model matching then turns into classification of the feature vector. More complex and more power- 
ful are 'relational models' that contain substructures and their interrelations such as distance and relative orientations. These informations are usually stored in graph-structures -- an approach that goes back to the early seventies /BARROW \& POPPLESTONE 171//BARROW et a1. '71/.

The matching of relational structures is known to be an NP-complete problem /SHAPIRO 179/. One must therefore keep in mind to use only graphs with few nodes or to partition the graph into subgraphs such as suggested in /BOLLES '79A/. Relational models can be constructed for binary images/HOLLAND '76/ as well as for gray level images/PERKINS $77 /$. In the first case, blobs are used as substructures, in the second case one typically chooses contour segments. In both cases one is well advised to include as much information as possible in the description of each substructure in order to facilitate the search through the model. For example, if local orientations of model elements are stored in the model, then the search for neighbouring elements can be confined to restricted areas in the image. Encouraging results have been obtained in the area of model driven analysis, yet there are many open questions and a lot of research will be necessary to make this approach applicable in a general and powerful way.

\subsection{EXAMPLES FOR PRACTICAL DIA-SYSTEMS}

In conclusion of the discussion of approaches from the literature we want to point to some specific examples that clarify the state-of-the-art. Early approaches for industrial applications have started in the seventies; as off the mid-seventies such applications are finding an ever increasing interest. The first approaches were typically simulations which did not perform in real-time, i.e. most often processes had to be slowed down or simulated completely. Also, these approaches were oriented towards very particular problems; only few approaches tried to tackle more general problems. Yet, with the development of this area, there is a strong tendency for more generality and we are seeing the first examples of systems that are commercially available.

In this section we will first give a survey on some approaches that seem rather typical. Due to the vast amount of literature a complete survey would be outside the scope of this paper, so we will more or less point to some 
articles. We will conclude this section with examples of DIA-systems that can be used in general ways with emphasis on those systems that are commercially available. As was pointed out in the introduction, important tasks for practical DIA-systems fall into the categories of visual inspection, handling, and control. In the literature, especially the first two categories have found a lot of interest.

Visual inspection and handling are two tasks that often go together for 2 reasons: parts that are to be inspected must be presented to the DIAsystem (often in a determined way); 2) if parts are presented to a DIAsystem for handling tasks one might as well inspect their quality (provided that the DIA-system is powerful enough).

Most inspection tasks are performed with binary images. These are not always obtained by thresholding but also by applying edge detectors to gray level images and then converting the results to binary images. Typical objects of interest for visual inspection are:

- Parts whose shape must be inspected, for instance

Screws where the shape of the thread can be determined from a silhouette which is easily obtained from binary images. Typical approaches work with the boundary of the silhouette or some derived representation. An example for the boundary approach is /BATCHELOR 178A/where the boundary lines are transformed into a chain code representation; an example for the second approach is /MUNDY \& JOYNSON '77/ where the median curve of the screw silhouette is first derived from the boundary lines and then used for syntactic recognition. Yet another approach works directly with the silhouette with a run length coding from which shape features can be computed /FLOSCHER \& PARTMANN ' 80 \%. It may be noted that /MUNDY \& JOYNSON '77/ use dedicated hardware and a minicomputer for on-line processing while /FLOSCHER \& PARTMANN ' 80 / use a general purpose DIA-system (to be described in the next chapter) which also allows on-line computation with high troughput.

Automotive parts where either parts have to be inspected before assembly or where assemblies have to be inspected for completeness. One example for part inspection is /PERKINS 179/ who checks control arm bushings with 
the aid of shape and spacing of notches that are on the outer rim of those bushings. The system is implemented in PL/I on an IBM 370/168 computer and uses gray level images as input. The circle boundary and the notches are obtained by applying the Sobel-operator for boundary detection. The extracted notches are then matched with those of stored models. As a result of the matching one obtains the class of the bushing as well as its orientation. An example for the inspection of assemblies can be found in /ALBRECHT et a1. ' $77 /$ who check steering gears. They work with binary images that are transferred to a minicomputer through a TV interface. Here, the authors check the presence of snap rings and similar components - - all checks being based upon shape analysis.

Tablets which must be inspected for roundness; in /NAKAMURA et al. '78/ and /NITTA '80/ we find an example where a DIA-system is applied that performs component labeling, as well as computation of area, perimeter, and centroid coordinates. This system could easily be applied in a number of different applications. The roundness of the tablets is obtained in this particular application through comparison between area and perimeter.

Labels on packages, bottles or other containers that must be inspected for correct size, shape, integrity as well as position and orientation. One early system performs heuristic checks along three selected scan lines that are binarized to verify label alignment /CALLEN \& ANDERSON 175/. A more complete analys is is performed in /BROOK et al. 177/, /CLARIDGE \& PURLL 178/ who use a linear diode camera for scans in the X-direction and the motion of a conveyor belt for scans in the $Y$-direction. The obtained binary image is further reduced by edge detection which is performed by special hardware. From the edge image one determines label orientation as well as pattern correctness. Due to the high data reduction high throughput can be reached despite the application of a micro computer for data analysis.

Parts where complex patterns must be inspected such as:

Printed wiring boards or mask patterns where a great many lines must be checked for indentations, protrusions, gaps, distance to neighbouring lines, etc. This application has found a very wide spread interest and 4 basic techniques have been developed for this type of inspection 
/JARVIS ' $80 \mathrm{~B} /$. These are: 1) non-reference methods which work with definitions of local shape and size and are the most popular techniques; 2) pixel by pixel comparison methods which are difficult to implement because of alignment and dimension problems; 3) local pattern matching techniques which look for correct or incorrect local patterns; or 4) comparison techniques based on symbolic descriptions. Examples for nonreference methods can be found in:/STERLING 19/, /EJIRI et a1. '73/, /GOTO et al. '78/ or /RESTRICK 177/; local pattern matching is performed in /JARVIS 18CA/; one example for model driven comparison can be found in /THISSEN $177 / 78 /$. Recently, syntactic models that describe mask patterns have found some interest.

Typical examples for the application of DIA-systems for part handling are:

Electrical probing of IC chips which requires to determine the exact position and orientation of the chips on an already assembled component. Based on relational template matching in gray level images the corners of the chip are found and the orientation of the chip is computed. This information allows to position electrical probes into the base and emitter areas of the chip for functional tests/BAIRD $176 /$, /BAIRD $178 /$.

Bonding of chips with similar requirements as in electrical probing since here, too, one must determine the exact location and orientation of the chips in order to control bonding. Two examples are: /KASHIOKA et al. "76/ who use local pattern matching for chip positioning; /HSIEH \& FU '79/ who scan along vertical and horizontal lines in the gray level image to determine mask orientation and location as well as the location of 2 bonding pads. The first system is partially implemented in hardware and works online while the second approach was simulated on a minicomputer.

Sorting of parts according to their type, position and orientation. Part sorting is an important task since for transportation and storage workpieces are often scattered in bins, boxes or other containers. Visually controlled sorting devices usually consist of 4 components: feeder system, vision system, handling device, and part deposit. It would be most feasible to grasp the parts directly from the bin but - with few exceptions the state-of-the-art is far from a solution of this task. There are a number of set-ups for part sorting. These use bowl feeders, chutes or 
conveyor belts as feeding system;vision is performed with dedicated hardware, most often in combination with minicomputers; typical handling devices are:selective channels, X-Y-tables, turntables, pick- $\&-p l a c e$ devices and industrial robots; the parts are deposited either directly into machines or into magazines or pallets. Let us cite a fex examples. /CRONSHAW et a1. '79/, /CRONSHAW et al. '80/ use a bowl feeder where in an escapement are mounted 2 linear bundles of fibre optics that provide slit views of passing objects in two directions. Recognition is based on point templates that are interactively constructed. In this experimental set-up no handling is provided.The combination of a bowl feeder and a $X-Y$-table that can be rotated is shown in /SARAGA \& SKOYLES 176/. Workpieces are fed onto the table which is in the viewing field of the camera. After location and orientation of a part are determined the table is moved and rotated such that the workpiece comes to rest at a fixed site. From there it is picked and transferred to a second fixed site - the deposit - by a pick\&-place manipulator. Visual recognition of the workpieces is based on models that contain features such as area, perimeter, polar radius, etc.

The idea to decouple handling actions into several separate steps is driven even further in the approach of /HILL \& SWORD '80/. The system consists of a belt or a vibratory feeder, a movable shuttle, an elevator, a turntable, a vision system and an industrial robot. Computer-controlled operations of elevator, shuttle and turntable can bring a part to a desired stable state, location, and orientation such that it can be grasped by the robot. Undesired stable states can be changed by pushing the part from the elevator at a predetermined height ('controlled tumbling'); desired orientations are obtained with the aid of the turntable. The shuttle moves the parts between elevator, turntable and pick-up site from where the robot moves the part to a pallet that is mounted on a $X-Y$-table. The set-up uses a vision system (Vision Module VS 100) that will be described later in this section.

One example for grasping parts from a conveyor belt with a robot is shown in /ZURCHER 178/. The vision system extracts the contour of the workpiece images, computes the centroid for part location and performs a polar coding comparison with a reference for part orientation. These data control the manipulator which grasps the parts from the belt. Another example for grasping of parts from a running conveyor belt will be 
explained in chapter 3 .

The use of a robot for part handling allows to grasp parts that abut or rest upon each other. This may happen when parts are fed from a chute and one example for an experimental set-up is / KELLEY et al.'77/. A binary image of the workpiece scene is obtained and local image features are computed from it. From these, feature candidates are selected for recognition of workpieces. The list of candidates is further refined by checking relations between features. Once a workpiece and its orientation are found it is verified whether that part can be grasped. If so, the robot acquires the part. Obviously this approach leads towards the "grasp from the bin". This problem is tackled in a different way by two similar approaches: / KELLEY at al.'79/ use a surface adapting vacuum gripper to grasp parts form the bin. One camera mounted on the robot arm guides the gripper by locating smooth surfaces where the gripper can get hold. Once a part has been grasped in a random position it is presented to a second camera. Here, the orientation of the part is determined and the robot can now place the part in a desired stable state at the goal site. A similar approach was used by /GEISSELMANN ' $80 /$ who uses a magnetic gripper instead of a vacuum gripper and deposits the part in front of the camera.

Assembly tasks are often performed with the aid of tactile sensing, for instance in bolt fitting to prevent jamming. Yet it seems useful to consider visual sensing as well to guide the manipulator approximately to the site of fitting. Visual control for assembly tasks has indeed been studied in a number of approaches. In an experiment where a rectangular block was inserted into a rectangular hole/TANI et al. 177/ the authors have mounted fiberscopes to the manipulator for image acquisition. From the binary image the contours of the bar and the hole are checked until they are parallel. Here, perspective distortions must be accounted for. In another approach the camera is mounted directly in the hand of an industrial manipulator/AGIN $777 \mathrm{~B} /$. The assembly task is to insert bolts into holes. First, an initial gross correction brings the robot arm (and the camera) from a random position to a position above a hole, then two fine corrections are perfomed. A similar assembly task is required in a related experiment by /MCGHIE \& HILL '78/. Here, the assembly operation consists of placing a cover on a compressor housing and fastening them together with eight bolts. The experimental set-up includes a robot, an 
$X-Y$-table, and DIA-system. The DIA-system analyzes binary images of the top of the compressor housing and commands the $X-Y$-table to move the compressor housing such that the cover can be placed. After each assembly step the DIA-system also inspects the result of the operation.

Yet another example is the approach of /OLSZTYN et al. 173/ where wheels are mounted by a visually controlled manipulator. In this experiment the DIA-system has to find studs on hubs as well as stud holes in wheels. The DIA-system first determines the center of symmetry of either hub or wheel and then uses this information to perform a circular search to locate the studs and the holes.

While the afore mentioned assembly tasks are rather simple and could be performed with acceptable efforts, the final example shows how difficult complex assembly tasks can be /KASHIOKA et al. 177/. The authors describe a multi-sensory robot which was tested in a vacuum cleaner assembly operation. The robot has two arms (a power arm and a sensor arm) and no less than seven cameras are applied.

From all these examples we see the variety of tasks that exists for DIAsystems. Until now, most often specific solutions of particular tasks have been sought and realized. Sometimes even only simulations or off-line computations were performed. Nevertheless there are some approaches that try to generate systems that provide more generality. These approaches can be grouped into several categories depending on their basic philosophy. These categories are:

\section{1) Software based systems: such systems usually store the image with the} aid of a fast interface either directly in the computer or in a dedicated image memory. The complete analysis is performed by accessing the stored data with software. Usually in such systems either micro or mini computers are used. Such systems are highly flexible and it is not surprising that they are used in research institutions that are envolved in basic research /BATCHELOR'78B/./BIRK, KELLEY et a1. '79 A, B/. Typically, such systems are based on binary image analysis - as for instance /PUGH et al. '78/ - while some systems store gray level images before they convert the picture to binary data /SPUR et al. '78/. At the time of writing only a few systems 
are manufactured by the industry and are available commercially. Among the few, one has to take notice of the VS-100 system which is sold by Machine Intelligence Corporation and is an off-spring of work that was performed at the Stanford Research Institute /AGIN \& DUDA 175/, /AGIN 175/, / AGIN 177/A/, /GLEASON \& AGIN 179/,/BOLLES '79B/. This system adapts to several cameras ( 3 solid state with resolutions varying from $256 \times 1$ to $240 \times 240$ and a standard vidicon camera). Its hardware basically consists of a binarization unit, a run length encoder with image memory, and a DEC LSI-11. Its software provides efficient programs for connectivity analysis, the computation of 13 features such as area, perimeter, centroid coordinates, number of holes, minimal and maximal radii,..... - nearest neighbour classifiers and a menu driven operation system, as well as 1/0-ports for communication with other devices such as controllers for gates, $X-Y$-tables, industrial robots, etc.. Typical performance times vary from $25 \mathrm{~ms}$ to $2.5 \mathrm{sec}$.

2) Hardware based systems: such systems process the image directly during the image scan. Most often only rather simple operations can be performed during the scan therefore methods such as pointwise template matching or the polarcheck are brought to bear /BRETSCHI 176/, /GEISSELMANN ' 80/.

3) Mixed systems: Certainly a very promising approach would be to mix software and hardware based approaches by applying hardware whenever many data have to be processed very fast and by applying software when data have to be analyzed in a flexible way. Two examples that use such hybrid approaches are /KARG 178/ and /KARG \& LANZ '79/ as well as /ARMBRUSTER et al. '79/ and /MARTINI \& NEHR '79/. In the first case a 2-processor system is applied where a LSI-11/2 provides the system management and a bit sclice microprocessor is used for fast access to an image memory. This system binarizes the image data on-line, stores them in 2 image memories and accesses these data through the $\mu \mathrm{P}$. In 2 processing steps features such as area, centroid coordinates, radii, area as a function of polar distance or polarcheck intersections are computed. The second system uses quite similar features; contrary to the first system special hardware is applied to compute area and centroid coordinates of a workpiece in the (binary) image.

4) Light section systems: the use of light section techniques delivers most of ten stable results - a fact that makes this technique rather feasible for industrial applications. Basically, images that stem from light sectioning 
illumination are easily reduced to binary images; therefore these systems are included in our survey. Such systems use either linear or 2-dimensional diode arrays for image acquisition. Examples for the first approach are: the CONSIGHT system /WARD et a1. '79/ that uses two strip lights and a linear diode array. Due to the presentation of workpieces on a conveyor belt the system acquires silhouettes of passing objects. These silhouettes are recognized with the aid of shape features such as area, first and second moments, or hole shape features. Much the same approach is pursued by /WOLF '79/ who determines two-dimensional features of the workpiece base as well as three-dimensional features of the workpiece volume (by triangulation). The application of such techniques to object tracking and welding seam following can be found in /AGIN $79 /$.

Two-dimensional approaches are also used in light sectioning techniques. Two examples are /VANDERBRUG et al. '79/ and /TODA \& MASAKI '80/. One essential feature of light sectioning is the fact that the images are easily converted to binary images. Thus, the same image analysis techniques apply. One particular advantage is that three dimensional information can also be obtained from the data.

In concluding it can be stated that existing systems are ready for many practical tasks that one encounters in the industry. At the time of writing the first systems are about to be commercially available and many more are likely to follow. It is generally predicted that these systems will find a wide spread use. 
In this chapter we describe a DIA-system that the authors have developed at the Fraunhofer-Institute for Information and Data Processing (IITB) at Karlsruhe. The system will be referred to as S.A.M. which stands for 'Sensorsystem for Automation and Measurement'. S.A.M. is commercially available from Robert Bosch GmbH, TV Equipment Division in Darmstadt, W-Germany.

Let us first explain the basic philosophy that guided our development. As was pointed out above, there is a wide range of practical tasks for DIA systems. Tasks with low complexity require simple measurements such as lengths, widths, etc. Highly complex tasks denand recognition of workpieces with a multitude of stable positions. Thus there is an extremely broad range of complexity that a DIA system has to cope with. The same holds for the required processing times: these vary from tenths of seconds to several seconds. It is obvious that no single DIA system - as universal as it may be - can cope in an economic way with these wide ranges of tasks and processing times. There may be simple tasks with long processing times allowed; here one would best apply a DIA system that performs the analysis in software from an image memory. On the other hand there may be difficult tasks that must be solved in a very short time; here, a lot of special hardware must be applied. Thus the costs of DIA systems also range from cheap systems to costly ones.

The answer to these problems are modular kits from whose modules DIA systems can be configurated such that they are cost effectively adapted to the task at hand. Thus one can always pick the most economic solution. One such modular kit is S.A.M. which is therefore not a DIA system itself; rather from its modules DIA systems can be built. S.A.M. consists of a number of hardware and software modules that perform an extremely fast analysis of binary images. 


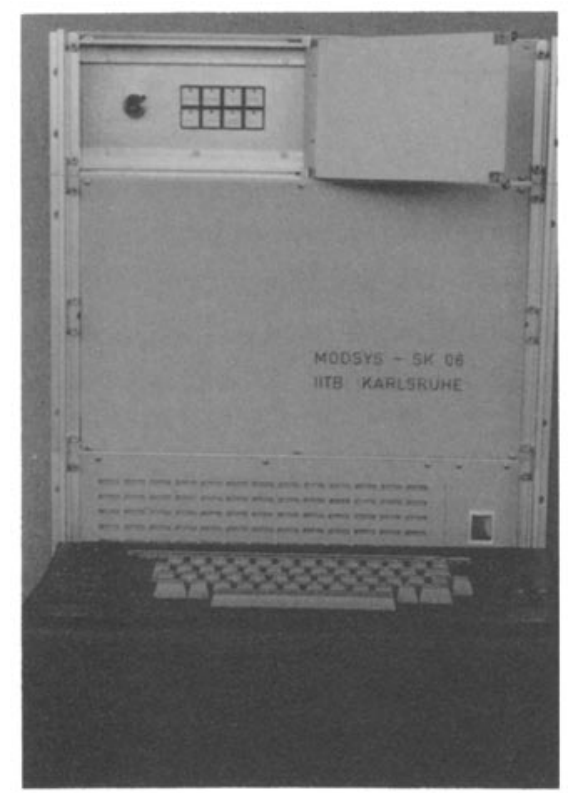

Fig. 3.-1: A typical S.A.M. configuration

Typical processing times are between 50 and $500 \mathrm{milliseconds.} \mathrm{S.A.M.} \mathrm{can} \mathrm{be}$ extended towards simple measurements as well as towards gray level image processing. From S.A.M. components one can build simple configurations as well as complex ones. Figure 3.-1 shows an example of a S.A.M. configuration. As can be seen there is only a keyboard and a panel of buttons for the operation of the system. With the panel one selects START, STOP as well as the display of one of four images on a TV monitor (analogue, binary, memory 1, memory 2). As will be explained later all operating modes of the system are selected with the aid of a menu driven dialogue. Thus the system can be operated by almost untrained persons. 
We might mention that S.A.M. was developed under the project name 'MODSYS' (for:modular system) and was renamed after completion. Therefore previous publications about our project all refer to MODSYS /FOITH et al. '78/, /FOITH 179/, /FOITH et al. '80/, /RINGSHAUSER '80\%, /ENDERLE '80/.

\subsection{S.A.M. - HARDWARE}

The most drastic problem in real-time image processing is the immense amount of data one has to cope with. For economical reasons the use of micro or mini computers is imperative yet if these were put to processing complete images, cycle times would certainly be outside the required ranges. It is therefore important to reduce the amount of data as much as possible. From our point of view the microprocessor never should get so "see" the complete image but only "interesting" parts of it or - even better - only data that were obtained from the image through dedicated processors. For this reason we have implemented into S.A.M. a number of features that support the reduction of data in various ways. These features make S.A.M. different from most other systems that we are aware of.

The most important features that serve to reduce data are:

- Iterative image processing capability where operators such as shrink, blow or combinations thereof can be applied consecutively to a stored binary image. The amount of necessary hardware is rather small due to a 'ping-pong processing' mode where the image is transferred hence and forth between two image memories. At each transfer the selected operator is applied at TV speed. 
- On-line image analys is where the following computations on a binary image are performed in parallel during the TV scan: 1) component labeling, 2) computation of area, 3) computation of perimeter, 4) computation of number of holes, and 5) computation of centroid coordinates. Steps 2) through 5) are performed for each labeled blob in the image (for up to 255 labels).

- Inversion of images from black to white and vice versa allows the computation of the above mentioned features for holes as well with the same hardware. During image analysis black pixels are considered to be figure points and usualiy one selects the polarity of the binary image such that black points correspond to regions. If one wants to analyze hole features as well, one scans the (stored) image a second time and inverts simultaneously its polarity. As a result, image analysis is now performed on the hole features.

- Suppression of uninteresting blobs can be performed by means of a filtering mode that is implemented within the component labeling module. One specific feature of this module is the fact that the label numbers are not stored pixelwise, in fact they are not stored at all. As will be explained later only the equivalences and the number of assigned labels are stored. If one needs a blob with a specific label one simply repeats the component labeling, only this time with the selected labels flagged. The filtering mode then suppresses all blobs whose labels are not flagged. Since component labeling is done with TV speed it oniy takes $20 \mathrm{~ms}$ for a half frame.

- Logical combinations of an input image with stored images help to reduce the incoming data by setting windows whose size and shape are determinded by blobs from a previous image. One can also combine input images with computergenerated patterns.

The overall structure of the system reflects our basic philosophy about data reduction. Since we intended the design of a modular system,S.A.M. had to be bus-oriented. The S.A.M.-Bus consists of two different subsystems: a videobus and a processor-bus. If one reads Fig. 3.1.-1 from the left to the right one notices 3 layers of processing units: 1) video circuits, 2) image processing and analysis and, 3) data processing and storage. Video circuits, the image processing and analysis units including image memories share the video bus; image analysis units and image memories share the processor bus with the $\mu \mathrm{P}$, data memories, and $1 / 0$ devices. Thus the image processing and analysis layer can be thought of as a reduction stage where the huge amount of image data is reduced to a few data that must then be processed by the data processing stage. In the following we will explain the important modules with greater detail. 


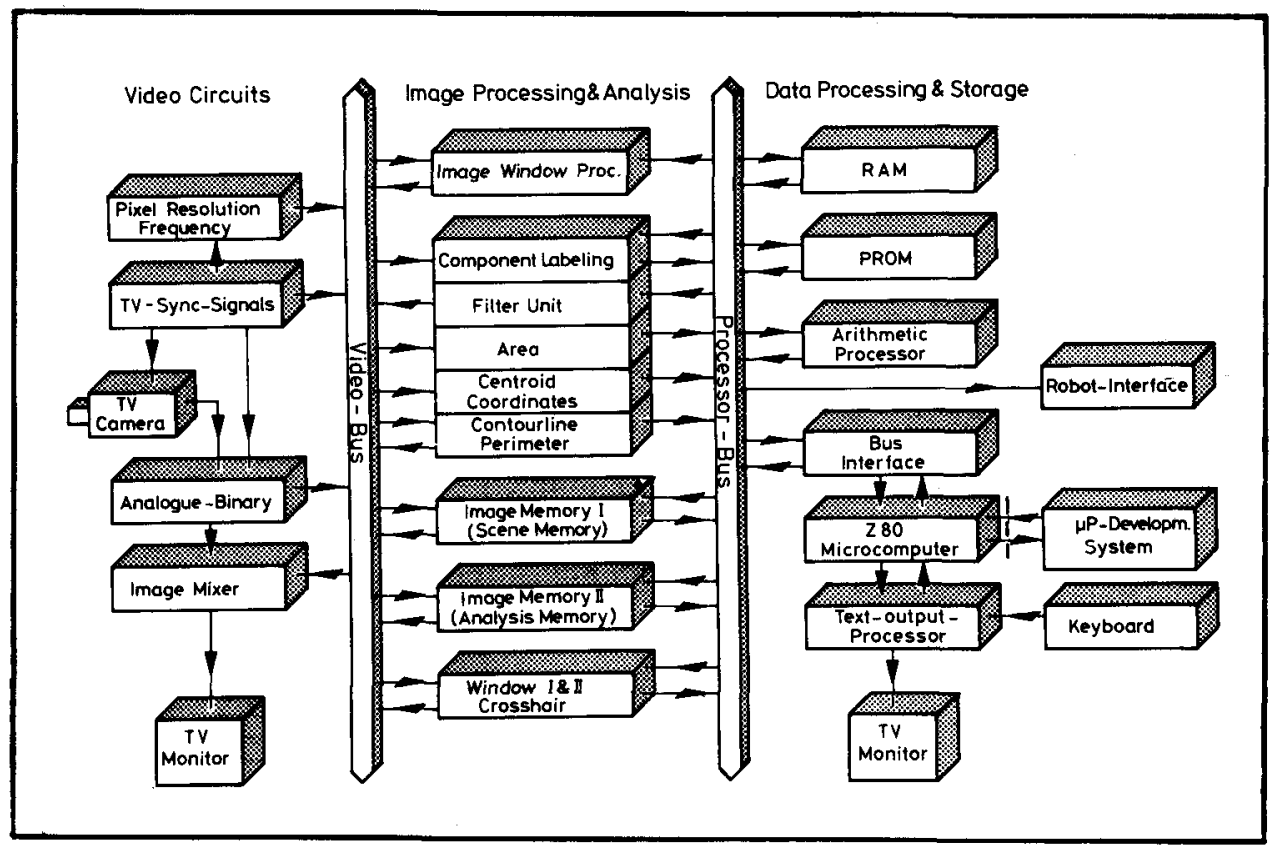

Fig. 3.1.-1: Blockdiagramm of S.A.M. hardware

\section{VIDEO CIRCUITS}

Video circuitry consists of Signa 1 Input Processors. (SIP) that perform the binarization and synchronisation of signals from either TV or solid state cameras. It is possible to read in images from 2 cameras simultaneously. The polarity of the input images can be selected (black/white or vice versa). The Pixel Resolution Frequency module determines the resolution along scan lines. In order to obtain square pixels when working with TV half frames we usually chose that frequency such that 320 pixels are resolved in one line. The Analogue-Binary module thresholds the image with a threshold that can be set by hand. In the future this module will be replaced by a module where threshold intervals can be set by the $\mu \mathrm{P}$. The Image Mixer module supports the simultaneous display of any combination of gray level image, binary image or images from two image memories. The binary images are coded with different gray levels as to be able to discriminate between them on the video monitor. In the same way two image windows and a crosshair are displayed. 


\section{IMAGE PROCESSING AND ANALYSIS}

The layer of Image Processing and Analysis modules consists of three different groups: image memories (including windowing and crosshair), image processing units, and image analysis units. Although memories are not processing devices they are included here because they play an essential role in the processing and analys is steps.

\section{Image Memories (IM)}

There are two types of Image Memories; a half-frame-IM and a run-length-IM. Both IM types can read/write with TV speed. The S.A.M. -Bus allows the application of up to 8 IMs of either type simultaneously.

The half-frame-IM stores the binary image pixel by pixel with a capacity of $256 \times 512 \times 1$ bit. Each pixel is addressed via its $X-Y$-coordinates. The $\mu \mathrm{P}$ can access every pixel and either read the bit or write it. The $\mu P$ also commands the half-frame-IM to: 1) start reading in an image, 2) start to output an image, or 3) to invert the image that it outputs. In the output mode the IM generates a binary video signal from the stored image. It is possible to link two IMs such that one outputs an image which the other one reads. Thus 'blob filtering' can be performed in cooperation with the component labeling module; the linkage of two IMs can also be used for the 'ping-pong processing' mode where the image is transferred hence and forth between the 2 memories while at each transfer some binary operation is performed.

The run-length-IM stores the positions of black/white and white/black transitions along scan lines with a capacity of $4 \mathrm{~K} \times 16 \mathrm{bit}$. This IM knows two different types of data words: transition words (TW) and line-number words (LN). Both types of words are 13 bit long. Bit 0 - bit 8 are used for the $X$-coordinate of a transition or the line number (=Y-position), respectively.Bit 9 specifies the polarity of the transition, bit 10 - bit 12 determine the type of data word (TW, LN, begin $1 / 2$ half frame, end $1 / 2$ half frame). When the run-length-IM reads in an image, it stores at the beginning of a line a line number word, even if the line is empty. Transitions along a line are stored with the corresponding TW. Thus this IM contains data in the following way (for some imaginary image): $\mathrm{LN} / \mathrm{LN} / \mathrm{LN} / \mathrm{TW} / \mathrm{TW} / \mathrm{TW} / \mathrm{TW} / \mathrm{LN} / \mathrm{TW} / \mathrm{TW} / \mathrm{LN} / \mathrm{LN} /$. The $\mu \mathrm{P}$ can access any stored word and can al so write into the memory. Run-length-IM commands are: 1) start reading half frame image, 2) start reading frame image, 3) start to output half frame image, and 4) start to output frame image. In the output mode the IM generates a 
binary video image from the stored data. This image can be displayed on a TV monitor. The run-length-IM can be applied in much the same way as the halfframe-IM. It is especially useful when run length features must be computed with the $\mu \mathrm{P}$. A particular feature is the capability to shift the stored image in positive $X$ and $Y$ direction deliberately by setting initial coordinates with one command.

It is not necessary to store always the complete TV images. In order to reduce image size two windows can be set which define the area of the image that is actually processed and stored. Window 1 defines the maximal area which is $256 \times 512$ pixels. Its left upper corner also defines the origin of all coordinates. This window is positioned by hand and cannot be accessed by the $\mu P$. The second window lies within the first and can be set by the $\mu P$ in size and position. Thus one can restrict the analysis to certain areas that one is interested in. The Window module also contains a crosshair generator. The crosshair is used to mark any position in the image. It can be used by a human operator or be positioned by the $\mu \mathrm{P}$.

Image Processing with the Image Window Processor (IWP)

Image Processing is performed with the aid of the Image Window Processor (IWP). This module represents a $7 \times 7$ image window that moves with the TV scan along the image. Binary operators are implemented that process pixels within the window through logical connections. Right now we have realized 8 different operators that can be selected by the $\mu \mathrm{P}$. These operators are: shrink, doubleshrink, blow, double-blow, shrink-and-blow, blow-and-shrink, contourline (= difference between shrink and blow), and finally NOP (= no operation). The IWP can also compare two images via XOR and AND. It can also mix one image into another.

Al1 operations can either be performed on TV-input and/or stored images. Output signals of the IWP constitute a binary video signal that can be routed to one of the image memories. Operators are realized by hardwiring. Any of the operators as well as the direction of the signal flow are determined by commands from the $\mu p$ that are sent to a data port.

If two image memories (IM) are used, iterative image processing can be performed in the 'ping-pong-processing' mode where the image is transferred hence and forth between the 2 IMs and is processed at each transfer. A11 IWP-operators work at TV speed. Thus one can apply image transformations many times without 
great loss of time. Usually such transforms are to clean the image by suppressing noise, closing gaps, or removing notches. Fig. 3.1.-2 shows an example for the iterative cleaning of a binary image (B) with the aid of the following sequence: double-shrink (C), shrink (D), and double-blow (E). The comparison of (B) with (E) shows that much of the noise has been suppressed while the figure of interest has kept its original shape. The whole sequence of transformations takes only about $80 \mathrm{~ms}$ ( $20 \mathrm{~ms}$ per transformation and a few ms for $\mu \mathrm{P}$ control).

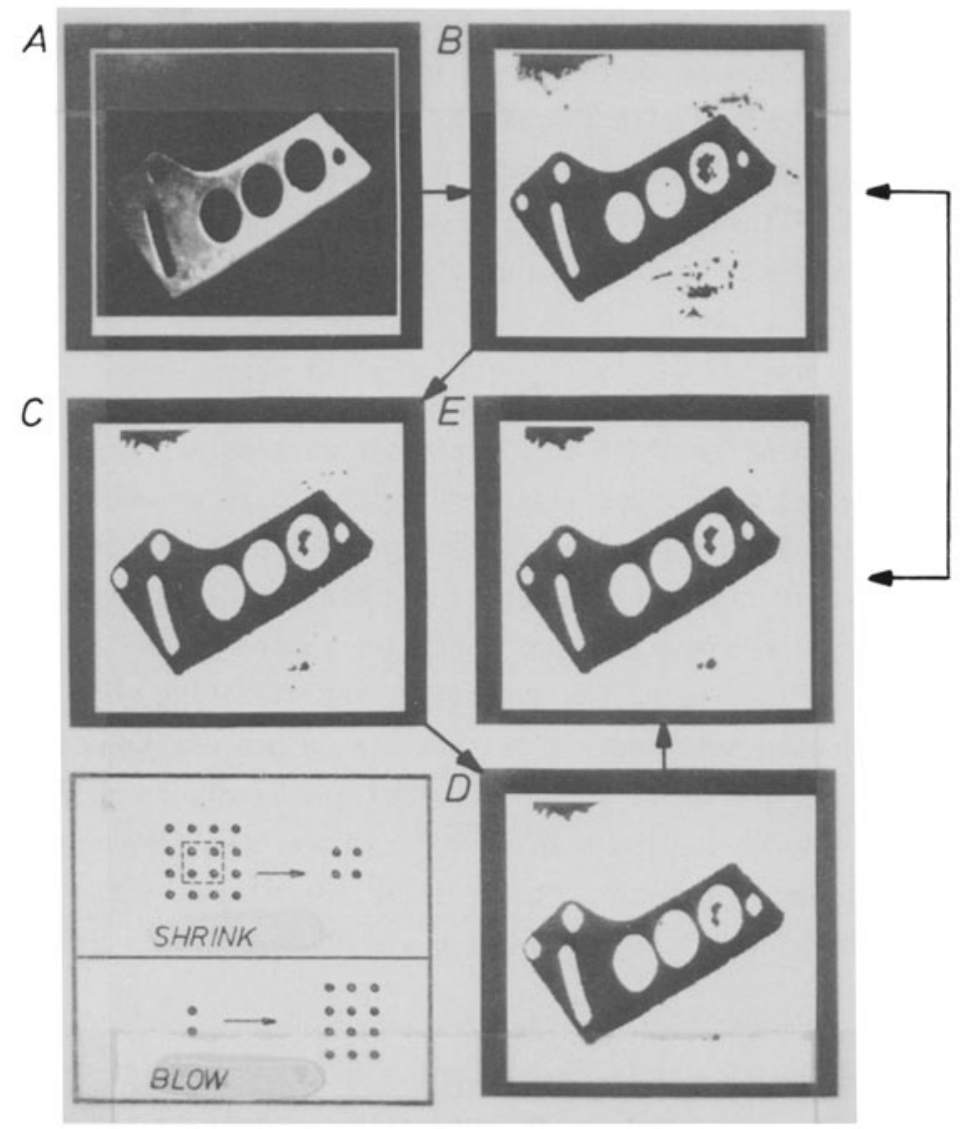

Fig. 3.1.-2: 'Clean-Up' By Iterated Picture Processing

$$
\begin{aligned}
& A \text { - TV Image } \\
& B \text { - Binary Image of } A \\
& C \text { - Double Shrink of } B \\
& D \text { - Shrink of } C \\
& E \text { - Double Blow of D }
\end{aligned}
$$


Image Analysis Processors (IAPs)

The Image Analys is Processors (IAPs) are the core of S.A.M. Basically, they perform: 1) component labeling, 2) computation of: (i) area, (ii) perimeter, (iii) number of holes, (iv) centroid coordinates for each labeled blob. It is the role of the IAPs to perform the necessary data reduction by extracting shape and position features from the blobs in the binary image. These features are the data that must be further analyzed by the $\mu P$. Let us briefly evaluate the reduction rate. A binary image with 2561 ines and 320 pixels per line has almost 100.000 bit. Suppose that such a binary image contains about 60 blobs. Each blob can be described with the aid of the extracted features by approximately 16 byte (cf. section 3.2), i.e. such a binary image can be represented by less than 1 kbyte. Although bit and byte operations may not be directly comparable it is obvious that the savings in data processing are tremendous and may amount to a factor of 1.000 .

We will describe the IAPs with greater detail in the following. It may be noted here that the component labeling module plays a particular role among the IAPs since it knows two different operating modes: the 'data extraction' mode is the actual process of labeling and the 'filtering' mode where the labeling process is repeated, yet this time certain labels are flagged. In this mode flagged labels (or rather, the corresponding blobs) are passed on while unflagged blobs are suppressed from the image. The purpose of the filtering mode is, again, data reduction. Due to this mode it is possible to process images such that the resulting images contain only those blobs that are likely to be of interest for a more scrutineous analysis. Such an analysis could then take place either with more dedicated hardware or with software in an image memory. The filtering mode is a powerful tool since it helps to cut costs in hardware as well as in software.

During the data extraction mode the component labeling module (CLM) determines connected components in binary images and assigns labels to these components. These labels are numbers that correspond to the order of appearance in the TV image from left to right and top to bottom (TV scan). The principle of connectivity is based on the observation of three consecutive pixels in the previous line (i.e. we use 8 -adjacency) through a $2 \times 3$ window:

$$
\begin{array}{ll}
\text { Line N-1 : } & \text { P P P } \\
\text { Line N : } & X
\end{array}
$$

If there was a label on any of the points $P$ on line $N-1$ then the same label holds for pixel $X$. If not - and if $X$ is a point of a blob - then a new label 
is assigned. Such an event corresponds to the beginning of a new blob; the first event corresponds to the continuation of an old blob. There are two more cases that one must take care of: convergences and divergences of branches of blobs. In the case of a convergence, two branches with different labels from the previous lines 'converge', i.e. it turns out that they belong to the same blob. In the case of a divergence one notices that one blob fans out into branches in the succeeding line. Since in this case one knows what is happening one can keep the old label for the new branches. In the case of convergences one must establish rules that determine which of the labels 'survives'. In our design we choose the leftmost label to be dominant over all other converging labels. Fig. 3.1.-3 shows examples for all these cases.

In the literature it is often suggested to store for each pixel the label of the blob to which it belongs. In practice this approach has two disadvantages. Firstly, one transforms a binary image into an 8 bit image (for 256 labels); secondly, if one wants to extract a connected component in a label image one must still search for all equivalent labels. We therefore don't store the labels at all. Instead, we store the binary image together with the equivalence list of labels and the total number of labels and repeat the process of component labeling whenever we are interested in a particular blob (or several blobs).

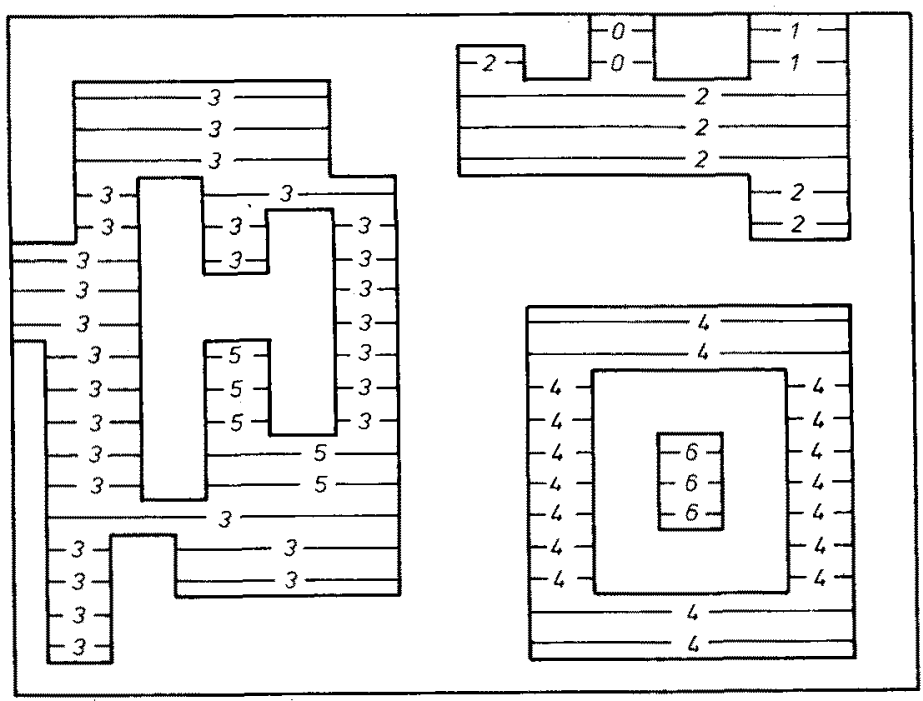

Fig. 3.1.-3 : The Principles of Component Labeling With Branch Beginnings, Continuations, Convergences, And Divergences 
Interesting blobs would typically be selected with the aid of features that were extracted during component labeling in the data extraction mode. The second run of the CLM is then performed in the filtering mode. Since results of the CLM are always obtained by repeating the component labeling we call this approach 'dynamic component labeling'.

Dynamic component labeling requires of course that there are processing units that are capable of computing features during the image scan since this is the only time when the labels are present. In fact, this is just what the IAPs do. As a result we obtain shape and position parameters that can be identified by their label number.

One particular feature of the CLM is the fact that it also determines if a blob is completely inside the image or not. If a blob touches the image window one cannot be sure how complete it is; therefore such blobs are marked and are later ignored in the analysis or treated separately.

The present realization of the CLM permits to assign up to 255 labels and as many convergences. If there are more blobs or branches in the image, then the first 255 are labeled by their corresponding number; the remaining ones are all labeled with label number 256. If the upper borders of blobs are fringed then a lot of labels would be assigned to those blobs. Therefore the CLM smoothes such borderlines by filling all branches that are only one pixel wide. Also, single points as well as holes of one pixel are removed or filled respectively. Due to this cleaning only relevant branches are labeled.

The results of the CLM allow to compute the number of holes of each blob. This will later be explained in the section on software. To conclude, the results that the CLM delivers are:

- the number of all assigned labels

: NAL

- the number of a11 convergences : NAC

- the list of convergences : CONVLIST

- the list of blobs that touch the image window: BORDLIST.

During the component labeling the CLM transmits the active label to the other feature processors such that the features of a blob can be assigned to its label.

The computation-of-area module (CAM) integrates for each label the number of pixels that belong to it. This number $A$ is given by: 


$$
A=\sum_{y=1}^{M} \sum_{x=1}^{N} B(x, y)
$$

where $x, y$ are the pixel coordinates; $N, M$ are the horizontal and vertical extent of the blob, respectively; and

$$
B(x, y)=\left\{\begin{array}{l}
1 \text { for pixels within the blob } \\
0 \text { for pixels outside the blob }
\end{array}\right.
$$

We assume here without loss of generality that the dimensions of pixels in $x$ and $y$ direction equal 1. The CAM has a counter that is loaded with the intermediate result of summation whenever the image scan moves across a labeled branch. The counter is incremented with the aid of the pixel resolution frequency at each pixel. When the scan leaves that branch on an line the intermediate sum is stored. After the image scan the area of each labeled branch is contained in the area memory. The memory can be accessed by the $\mu P$. Since a blob may consist of several branches it is necessary to add up all the partial areas. This is done at a later stage by software.

The computation-of-perimeter module (CPM) detects and counts contour points of blobs. A blob point is said to be a contour point whenever at least one of the neighbouring points belongs to the background. The detection is performed in a $3 \times 3$ neighbourhood around each blob point. For the computation of the perimeter it is not sufficient to integrate all contour points since the perimeter depends on the orientation of the blob on the grid. Contour points must be weighted according to the number of neighbours outside the blob. Yet, the error that one obtains when rotating a straight contour over the grid may be as high as $\pm 6 \%$. This error can be further reduced if direct and diagonal neighbours are weighted differently. The error is minimized to $\pm 4 \%$ when the ratio between direct and diagonal neighbours is set to 10:7. The CPM is realized with three stages of adders. First direct and diagonal background pixels are added up separately and weighted with multipliers. Then these sums are added in the second layer. This result is added to the perimeter that has been computed up to that point. The result of this adder is finally stored in the perimeter memory. The CPM can also be used to output the contour line simply by issuing a11 detected contour points.

The computation-of-centroid module (CCM) is the last IAP. For the computation of the centroid coordinates one assumes that each blob point has a mass equal to 1. Centroid coordinates are then: 


$$
x_{c}=\frac{\Sigma x \cdot B(x, y)}{\Sigma B(x, y)} \quad, y_{C}=\frac{\Sigma y \cdot B(x, y)}{\Sigma B(x, y)}
$$

Since $\Sigma B(x, y)$ is the area of the $b 7$ ob it is already computed by the CAM. It therefore suffices to compute the noninators of $(3,1,-3)$. The division by the area is later performed by software. The CCM works in much the same way as the CAM, the only difference is that coordinates are integrated instead of pixels.

As was already mentioned the CAM, CPM, and CCM receive the actual label number from the CLM and can thus store the results of their computations under the corresponding label number.

\section{DATA PROCESSING AND STORAGE}

There are three functional groups of modules in this layer: data processor units, data storage units, and data input/output units.

\section{Data Processors (DPS)}

Right now there are two data processor units (DPs). These are a $Z-80$ single Board Computer and a fast arithmetic processor (AM 9511) that support the $Z 80$ in numeric tasks.

The arithmetic processor has an 8 bit bidirectional bus, a data stack, and an arithmetic unit. First the two operands are pushed onto the stack then a command word is transmitted that specifies the operation. A status word signals when the operation is finished. Then the result can be read from the stack. The arithmetic processor performs 16 bit as well as 32 bit integer and floating point operations.

The $Z 80$ is the main controller of all system functions. It can send commands to IAPs. As can be seen from Fig. 3.1.-1 it is not directly linked to the processor bus. This is due to a special feature of the S.A.M.-Bus. When we designed the system we wanted to be able to apply 8 bit processors as well as 16 bit micro processors. We also wanted a lot of address space to be able to store tables, data and programs. The storage of tables can drastically support realtime algorithms by providing fast table look-ups. The processor bus has therefore a 16 bit wide data bus and a 24 bit wide address bus and a Bus Interface is needed. This interface has two functions: it links the $\mu \mathrm{P}$ to the bus and it provides a paging system for data storage access. It is possible to address 
256 memory pages with 32 KByte each. The $64 \mathrm{~K}$ address space of the 280 is divided (with the aid of the interface) into a direct part with $32 \mathrm{~K}$ and a paging part with the afore mentioned 256 pages of $32 \mathrm{~K}$. The address space of the paging part is 8 MByte.

\section{Data Memories (DMs)}

S.A.M. provides RAMs and EPROMS. EPROM boards offer a storage capacity of $32 \mathrm{~K}$ each; RAM boards have $16 \mathrm{~K}$ each. Depending on the S.A.M.-configuration one can use several boards of both types. All memories can be switched to either direct or paging access mode. For mass storage a floppy disk drive is used.

\section{Data Input/Output Units (DIO)}

Data input/output occurs on two different leveis: 1) the human operator must be able to communicate with the system, 2) the system must send and receive data from other devices of the workplace. For man-machine communication a commercial text-output processor was chosen (SGS/ATES VDZ80) that displays alpha-numeric data on a video monitor and receives alpha-numeric data from a keyboard. Thus the human operator can communicate with S.A.M. by dialogue driven techniques. The operator is further supported by a crosshair with which he can point to the image.

Data communication between other devices and S.A.M. can be performed by using the Z80 PIO and SIO. Specific data output channels are further: 2 DigitalAnalogue-Converters and a digital Robot Interface. This interface is adapted for industrial robots such as the Volkswagen R-30 or the KUKA IR-601. It delivers data for: a 'ready' message, the stable position, location and orientation of a workpiece; it can receive an acknowledge signal from the robot.

As was pointed out in section 3. these hardware modules can be used to build configurations of various complexity. Some typical configurations will be discussed in a later section.

\subsection{S.A.M. SOFTWARE MODULES}

Real-time processing of images cannot only be based on the application of dedicated hardware. It is also necessary to implement algorithms that process the data as effectively as possible. There is no systematic approach how to 
implement real-time processing algorithms. Yet, there are at least two general principles that facilitate real-time implementations: 1) the use of table-lookups instead of on-line computations; 2) presorting of data into highly organized data structures which are easily accessed. The use of table look-ups is supported in S.A.M. through the huge address space of the system; the organization of data will be explained in this section. Due to the implemented software it is for instance possible to perform a model-driven search in less than $200 \mathrm{~ms}$.

S.A.M. software is implemented in PLZ, a PASCAL-1ike language with two levels: an assembler type level (PLZ/ASM), and a high level (PLZ/SYS). According to the required speed we have either used PLZ/ASM or PLZ/SYS.

S.A.M. software is organized into a hierarchy of three layers. These layers are:

- basic software

- problem-oriented software

- operator-oriented software.

The first two levels are supposed to be used by the expert programmer who adapts a S.A.M. configuration to a particular problem. The third level is an interactive surface for the untrained operator who performs in-site programming at the workplace.

The basic software itself consists of two levels:

- a microprogramming level for hardware and $\mu \mathrm{P}$ control

- a higher level for collection, organization, storage, and access of data.

At this level one also finds some high system commands such as text $I / 0$, crosshair commands or graphic output.

On the second level are implemented routines for nearest neighbour classifiers, polar check, model driven search or other problem-oriented programs. It is at this level that a S.A.M. configuration will be programmed by a system programmer. The third level finally provides interactive means to operate the system at the workplace - for instance to adapt it to new workpieces. Here, no programming 
knowledge is required and the user is guided by menu driven dialogues.

BASIC SOFTWARE : MICROPROGRAMS

Let us discuss these levels at some greater detail. The basic software consists of two subsystems: MONSYS and SAMOS. MONSYS is the monitor program for the Z80; SAMOS is the control system for all S.A.M. hardware (besides SAMOS there are some more hardware control commands that are on a higher level then SAMOS commands but are still part of the basic software).

\section{MONSYS commands are grouped into:}

- Memory commands such as:

- display.m : displays the content of a memory whose address range can be specified.

- SET.M

: sets the content of a memory cell with a value that can be specified.

- FILL.M

: sets the content of a memory sector with a value that can be specified; also, the range of the memory space can be determined.

- MOVE.M : moves the content of one memory sector to another one.

- locate.s

: locates a string in a given memory sector and outputs its address.

- Register commands such as:

- display.r

- SET.R
: displays the content of register $R$.

: sets the value of register $R$.

- Break commands such as:

- SET.BREAK.A : sets a break point at address A.

- clear.break. a clears a breakpoint at address A.

- CONTINUE.B : clears a breakpoint, sets a new one, and executes the program from the new breakpoint ('GO').

- PROCEED. $B \quad:$ allows to continue a program while leaving the breakpoints where they are. 
- Execution commands such as:

- NEXT.N

- $\mathrm{GO}$

- JUMP.A
: executes the next program line(s) and displays the register contents.

: executes a program whose starting address is specified in the Program Counter.

: jumps to address $A$.

Floppy Disk Drive commands such as:

- SET.SECTOR

: moves a file to a sector of the floppy disk.

- GET.SECTOR

: transfers a file from the floppy disk to a RAM.

Port commands such as:

- OUT.P

: transfers data to a port (out of 256 ports).

- IN.P

: gets data from a port.

All S.A.M. hardware modules can be accessed through ports; thus one could program a S.A.M. configuration with the aid of these commands. Yet, this would be rather aukward since there is a special monitor system for the hardware, too: SAMOS.

SAMOS commands are grouped into:

Initialization commands such as:

- INIT : initializes the complete system including the filter memory in the component labeling module and all image memories.

- WINDOW : sets the image window to 'on/off' and - in the 'on'-case - to $x_{\min } / y_{\min }$ and $x_{\max } / y_{\max }$.

- EXEC

: executes a SAMOS command line.

Data extraction commands such as:

- READ.I

: reads an image while specifying the flow of information: it is determined to which image memory the image is sent and also which IAPs are to be applied.

- COMP.M

: performs the same operation as READ.I, only 
in this case the image is already stored in one of the image memories; therefore one must specify from which IM the image must be read.

Mode selection commands such as:

- fILTER. B

: filters blobs with the aid of the component labeling module (CLM); one must specify:

1) the direction of the image transfer (from memory 1 to memory 2 or vice versa), 2) the desired label numbers must be flagged.

- PROC.I : in this mode the Image Window Processor (IWP) is programmed. Again, the flow of information (either from TV, memory 1 or memory 2) as well as the operator sequence must be determined. It is possible to input any sequence of operations; the system completes the sequence always such that the resulting image will be in memory 1.

Interactive commands such as:

- xhair

: turns the crosshair on or off and also allows to either set it to specific coordinates or read the coordinates of its locations.

- get. CHAR : gets an alpha-numeric character from the keyboard.

- pUt.CHAR

: puts a character onto the TV-monitor.

- CONV.H.D

: converts a hex number to a decimal number.

- CONV.D.H : converts a decimal number to a hex number.

Graphic commands such as:

- LINE

- cirCle

- patTern

- ASCII
: outputs a line with given direction and coordinates.

: outputs a circle with given centroid and radius.

: outputs 6 different pattern (rectangle, diamond, cross, star, point, ....).

: outputs any ASCII symbol in a white field in an Image Memory. 
- Numeric commands such as:

- PUT.ARI : programs the arithmetic processor (ARI) by sending data and the required operation.

- GET.ARI : gets the data from the ARI when its status signal indicates that the operation is finished.

These commands control the complete S.A.M. hardware. As a result one obtains a number of feature data that can be used for a compact description of the binary input image. As was pointed out at the beginning of this section it is feasible to organize these data such that they can be retrieved efficiently. This organization is performed at the second level of basic software.

\section{BASIC SOFTWARE : DATA COLLECTION AND ORGANIZATION}

The first step at this level consists of the collection of all feature data into the "Scene Table" (ST) - a data structure onto which all further analysis is based.

After input of an image the Image Analysis Processors (IAPs) have stored the extracted features for all labels that were assigned to blob branches. Since a blob can consist of several branches, the features of the corresponding labels are only partial results. One must therefore determine the set of labels for each blob and combine their feature values into the final values. Let us call this process 'label collection'. It is performed by an algorithm that analyzes the convergence list (CONVLIST) (cf. section 3.1.).

If CONVLIST is empty then all blobs in the image have only one label each and the Scene Table can be generated directly. Otherwise, equivalent labels are grouped together by a fast algorithm in one run through CONVLIST. This algorithm works with a set of stacks into which equivalent labels are pushed. For fast access of the stacks a stack-address field (SA field) is used that contains as many cells as there are labels. The SA field is accessed directly through the label number. Every time a label gets pushed onto one of the stacks, a pointer to that stack is set in the corresponding SA cell.

Let us give an example (cf. Fig. 3.2.-1). Suppose the first equivalent labels $(i, j)$ have been pushed onto stack 1 . Then we set $S A(i)=S A(j)=$ 'pointer to stack 1'. If later another label turns out to be equivalent to either $i$ or $j$, 
then it gets pushed onto the same stack and we set the pointer in its SA cell to that stack. In our example, labels $k$ and 1 end up in stack 1 . In genera 1 , every time a pair of labels $(i, j)$ is taken from CONVLIST, one first tests whether one of the corresponding $S A$ cells already contains a pointer to a stack. The following cases can occur:

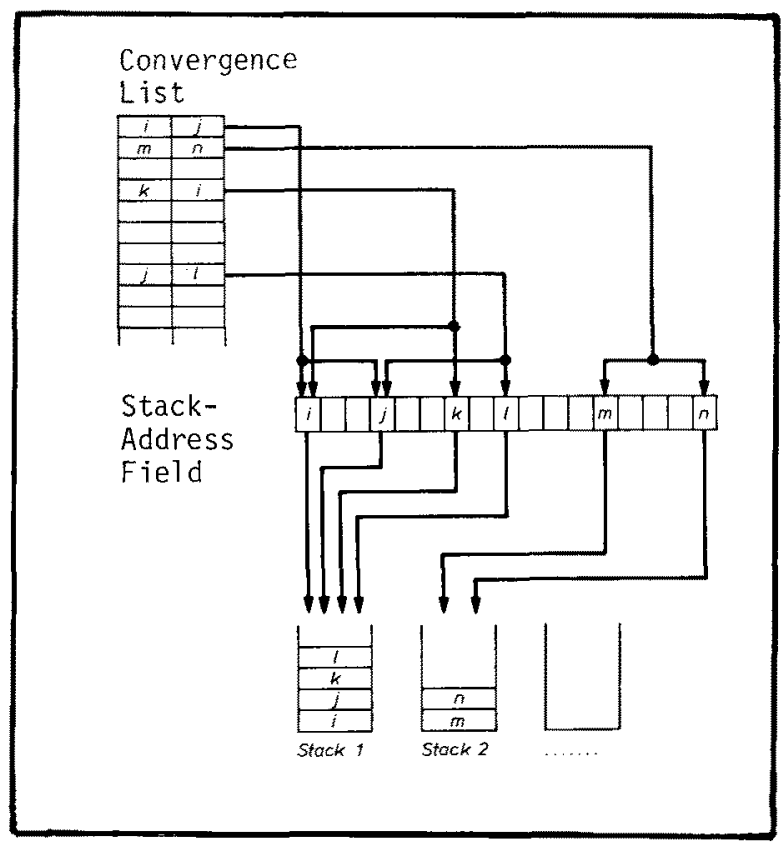

Fig. 3.2.-1 : Label Collection with The Aid of A Stack-Address-Field

1) None of the labels is in one of the stacks $(S A(i)=S A(j)=N O N E)$, then both labels are pushed onto the next empty stack $X$ and $S A(i)=S A(j)=$ 'pointer to stack $X^{\prime}$ are set.

2) One of the labels -- say $i$-- is already in a stack; then $j$ is pushed onto the same stack and we set $S A(j)=S A(i)$.

3) The two labels are in different stacks $(S A(i) \neq S A(j))$; then one the stacks is pushed on top of the other in order to collect all equivalent labels. At the same time, all SA cells that pointed to the emptied stack must be updated to the common stack and the empty stack is free again.

After one run through CONVLIST, each stack contains a set of equivalent labels. During label collection it is also noted how often each label appears in CONVLIST (1abel frequency is needed for computation of the number of holes in 
a blob). Due to the SA field the algorithm for label collection is linear in the sense that it performs with $O(N A C)$ where NAC is the length of CONVLIST.

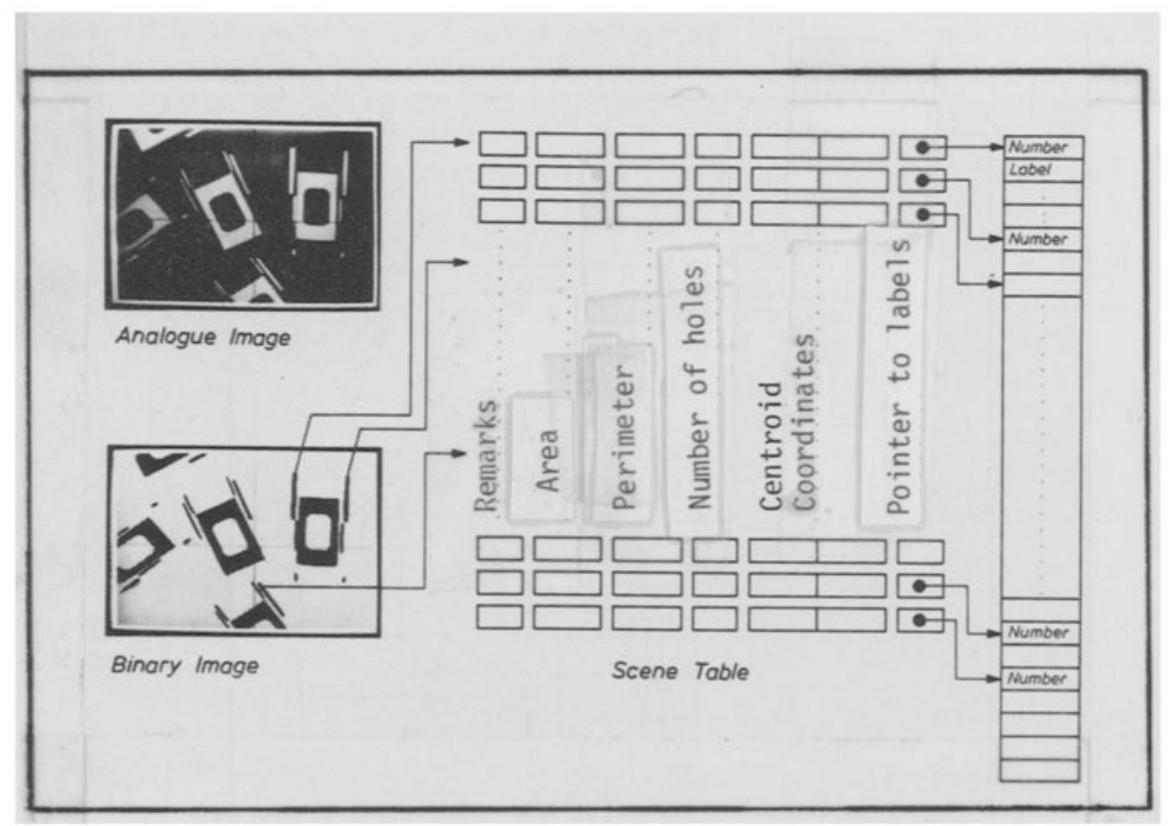

Fig. 3.2.-2: The Scene Table As A Compact Description of Binary Images

After label collection it is possible to generate the Scene Table (ST).

Fig. 3.2.-2 shows the structure of this table. The ST is a compact description of the binary image. For each blob there is one line in the ST; blobs are partialiy ordered from top to down in the order of their appearance during the TV scan. For each blob the ST contains (stored in 16 bytes): 1) a cell for remarks; 2) area; 3) perimeter; 4) number of holes; 5) centroid coordinates (after division of the nominator by area); 6) and a pointer to a separate record that stores corresponding labels.

During construction of the ST one must consider whether a blob has one or several labels. This can easily be determined with the aid of the SA field. We have two cases:

1) If a SA cell is equal to zero, then the corresponding blob has only one label. Its features can be directly entered into the ST where the number of holes equals zero. Actually, before the entry is made, it is verified that that blob doesn' $t$ touch the image window. This is easily done with 
the aid of BORDLIST (cf. section 3.1). If the blob touches the window, then no entry is made, i.e. it is excluded from further analys is since it is likely to be incomplete.

2) If the $S A$ cell contains a pointer to a stack (SA (i) $\neq 0$ ), then we have encountered a blob with several labels. After checking the BORDLIST for these labels we pop label by label from the stack and compute the final feature values by summing up all areas, perimeters, centroid nominators, and label frequencies. If a blob has NL labels with a total label frequency LF then the numer of holes can be computed by:

$$
\text { number of holes in a blob }=L F-N L+1
$$

After all computations, the data are entered in the ST as in case 1). Instead of suppressing blobs from the ST that touch the image window, it is possible to enter them and mark the ST line especially. Further more, blohs whose area is under a given threshold can also be suppressed form the ST ('software filtering'). During generation of the table total area, perimeter, and number of holes in the image are computed. This computation is performed in the Z80 while waiting for centroid coordinates from the Arithmetic Processor. These total features can be used to determine whether an image is likely to contain reasonable blobs at all.

As was aiready mentioned, the Scene Table contains blobs in a partial order from top to down. In image analys is one is often interested in two-dimensional range queries such as: "Is there a blob within the area: $x$ min/ymin and $x m a x /$ ymax?", or "what neighbours does a blob have?" Such queries can support the analysis very efficiently. Due to the partial order of the ST they cannot be easily performed in that table. It was therefore necessary to generate a second data structure which is organized in a two-dimensional way such that range queries are easity performed. We call this second data structure the "Scene Sketch" (SS).

The Sketch is simply a grid of $20 \times 16$ square cells that is laid over the image. Each cell covers a $16 \times 16$ subimage (given an image with 256 lines and 320 pixels along the lines). With each cell is associated an array of 4 words. Into this array all blobs are written whose centroids fall into the corresponding SS cell, This array is organized in the following way:
1) number of blobs in the cell
2) ST line number for first blob
3) ST line number for 2 nd blob
4) ST line numer for 3 rd blob or pointer to an overflow list. 
This organization allows efficient storage since there are seldom more than three blobs within the area of a SS cell. If there are more than three blobs in an SS ce11, then these can be found in the overflow list.

The address of an SS cell is obtained from the X-Y-coordinates of blobs by setting the high byte to $x / 16$ and the low byte to $y / 16$. Thus, if one wants to know to what cell a blob belongs to one performs 4 right shifts on its centroid coordinates and adds the results to the basic address of the SS. Elements in the associated array point to the line in the ST where the features of the corresponding blob are stored. The Scene sketch facilitates a highly organized access of the ST. It is generated from the Scene Table in one run through the table by the program SKETCH.SORT which performs with O(STL) where STL is the length of the Scene Table.

There are three basic search routines that work on the SS. These are:

\begin{tabular}{|c|c|c|c|}
\hline & SKETCH.SEARCH.C & : & $\begin{array}{l}\text { input are } X-Y \text { coordinates; } \\
\text { output is a list of ST line numbers of } \\
\text { those blobs that are in the same SS cel1. }\end{array}$ \\
\hline & SKETCH.SEARCH. 9 & $:$ & $\begin{array}{l}\text { input are } X-Y \text { coordinates; } \\
\text { output is a list of ST line numbers of } \\
\text { those blobs that are either in the same } \\
\text { SS cell or in any of the neighbouring } \\
\text { cells (i.e. } 3 \times 3 \text { SS cells are searched } \\
\text { for blobs). }\end{array}$ \\
\hline & SKETCH.SEARCH.W & : & $\begin{array}{l}\text { input are the coordinates of the upper } \\
\text { left and lower right corner of a } \\
\text { rectangular search window: } x \text { min/ymin and } \\
\text { xmax/ymax; }\end{array}$ \\
\hline & & & $\begin{array}{l}\text { output is a list of ST line numbers } \\
\text { of all those blobs that are in any of } \\
\text { the SS cells that the window covers. }\end{array}$ \\
\hline
\end{tabular}

With these search routines it is possible to start at some coordinates (not necessarily those of $a b l a b$ ) and find all blobs that are in the neigbourhood. That neighbourhood is either one SS cell, 9 SS cells, or all SS cells covered by the rectangular search window.

other search routines are based upon the organization of feature data since range queries about feature values are equally important ("Which blobs have an area between value-1 and value-2?", ...). Right now we only sort area values since range queries with this feature are often sufficient to reduce the number of likely candidates in an efficient way. 
Area values are organized into a data structure that consists of two substructures: the AREA.KEY-TABLE and the AREA.LIST. The AREA.KEY.TABLE (AK-Table) allows fast access to the data that are stored in the AREA.LIST (A-List). This is a technique that we often use: to have an access structure (mostly based on hash coding techniques) and another structure (mostly a linked list) that contains the sought data.

For accessing the AK-Table we have divided it into 6 ranges with increasing widths: $0-255 / 256-1023 / 1024-2047 / 2048-4095 / 4096-8191 />8192$. Each of these ranges is subdivided into a number of cells; the number of cells varies in order to represent each range according to its importance. Thus the ranges have the following cell distribution:

1) range $0-255: 16$ cells with 16 values each;

2) range $256-1023$ : 24 cells with 32 values each;

3) range 1024-2047: 16 ce11s with 64 values each;

4) range 2048-4095: 16 cells with 128 values each;

5) range 5096-8191: 16 cells with 256 values each;

6) range > 8192: 1 cell for all the remaining values.

We thus have the following structure for the AREA. KEY.TABLE and the AREA.LIST:

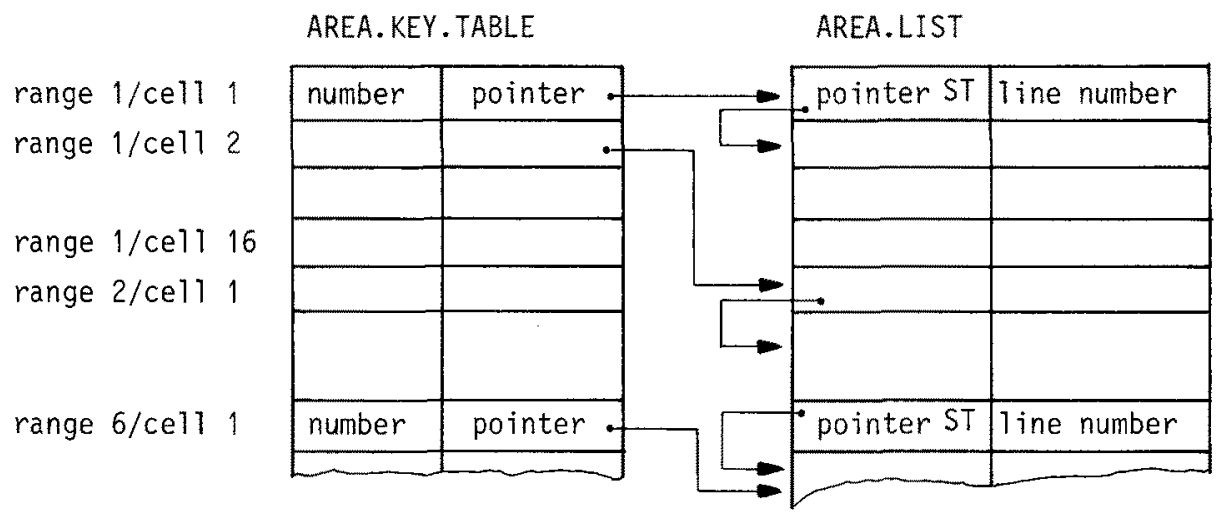

Thus, cells in the AK-Table indicate how many areas belong to that cell and the pointer indicates the starting address of the corresponding part of the A-List. A-List elements are then linked together by another sequence of pointers while the content of an A-List element is the ST line number in which the corresponding area value can be found. Both data structures are generated from the Scene Table by a program AREA.SORT which performs with 0 (STL), i.e. 
the structures are generated in one run through the Scene Table.

Based on the organization of area values two search routines have been implemented:

- $\quad$ aREA.SEARCH

- area.search.tol
: input is an area value;

output is a sequence of ST line numbers where area values of the according range/ cell can be found.

input is a deliberate range of area values: amax and amin;

output is a sequence of ST line numbers where area values can be found that are within the given range.

Based on the afore mentioned data structures we have implemented several Image Analysis Routines that are rather general in the sense that they perform data queries that are useful in many ways. Of particular interest are:

- PNT.PNT.DIST.DIR ; delivers the coordinates of a pixel if given the coordinates of a starting point together with a distance and a direction. input: $(x 1, y 1)$, distance, direction; output: $\left(x_{2}, y_{2}\right)$.

- DIST.P1.P2

- DIR.P1.P2

- FIX.TOL

- PERC.TOL

- SEARCH.WINDOW
: computes the distance between two given points.

input: $(x 1, y 1),(x 2, y 2)$;

output: distance $d(P 1, P 2)$.

: computes the direction between two points P1, P2 where the direction is defined to be the angle between the connection line (P1,P2) and a horizontal line through P1. input: $(x 1, y 1),(x 1, x 2)$ DIST; output: $P(P 1, P 2)$.

: computes a fixed tolerance for a given value of a feature.

input: feature value $v$, threshold $\delta$; output: vmin, vmax.

: computes tolerances in $\%$ of a feature value. input: feature value $v, \%$ tolerance; output: vmin, vmax.

: determines a square search window of given dimension around a point of given coordinates. input: $(x, y)$, width; output: $(x \min / y \min ),(x \max / y \max )$. 
- XHAIR.SELECT : compares a given crosshair coordinate with entries in the Scene Table; if the centroid coordinates of an entry coincide (within a specific range) then the corresponding ST ine number is given. input: $x-y$ coordinates of the crosshair; output: either an ST line number or ERROR.

All these programs constitute the level of basic software onto which the remaining two levels are based. They both make intensive use of these programs, and it should be noted here that it is the basic level whose routines do facilitate real-time processing of the data.

\section{PROBLEM ORIENTED SOFTWARE}

Problem oriented software performs the actual recognition tasks -- for instance in workpiece recognition and inspection. At the time of writing we have implemented three programs that can be used for quite a number of applications. For other applications it will be necessary to implement more programs. Due to the software that is available at the basic leve1, system programming is rather easy. In the following we will describe some procedures as wel1 as the three recognition programs.

The most basic step in recognition programs is the comparison between model features and blob features from the Scene Table. Typically a model determines what features a blob should have and it must be verified whether an actual blob has features that match the description. Since the S.A.M. hardware extracts area, perimeter, and number of holes, the procedure 'FEATURE. VERIFICATION" sequentially compares these features between a given model and all blobs in the Scene Table:

procedure 'FEATURE. VERIFICATION'

begin GET.MODEL. FEATURES

AREA.SEARCH. TOL

do AREA.LIST.NEXT

PERC.TOL. PERIMETER

PERIMETER. COMPARISON

od

do PERIMETER.LIST.NEXT

HOLE. COMPARISON

end 
As can be seen from the listing, the procedure first gets the model features and performs then AREA.SEARCH.TOL which delivers a list of blobs whose area values are similar(within a given range) to the model area value (AREA.LIST). In the next loop this list is checked for blobs whose perimeters are similar to the model perimeter. As a result one obtains another 1 ist (PERIMETER.LIST) whose elements are compared with a minimal and maximal number of holes of the model. The result of the complete procedure is a list of all those ST line numbers that contain blobs with verified features. It may be noted that before calling this procedure in a main program, procedure AREA.SORT must be applied which organizes area by increasing values.

One application of this procedure can be found in a 'NEAREST-NEIGHBORCLASSIFIER' program that delivers all blobs that correspond to a given set of input classes. These classes have been learned by the system during a 'TeachIn' phase that will be described later.

program 'NEAREST-NEIGHBOR-CLASSIFIER'

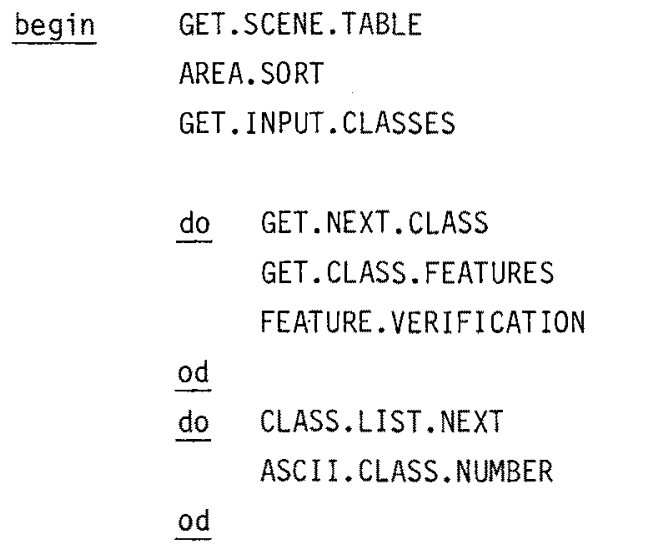

end

In the first step the Scene Table is constructed and the areas are sorted. Then a list of classes that must be found is obtained from a data memory. The procedures GET.NEXT.CLASS and GET.CLASS. FEATURES pass on model features to the FEATURE. VERIFICATION procedure which delivers a CLASS. LIST for each input class. Each such CLASS.LIST contains matching blobs. In the final step a 11 recognized blobs are displayed on a TV monitor together with their class number (ASCII. 
CLASS, NUMBER).

Previous to a discussion of the second recognition program 'POLAR.CHECK' we must briefly explain a data structure that is very similar to the AREA. KEY. TABLE/AREA.LIST structure. This structure is called MODEL. ACCESS. STPUCTUIDE and it is shown in Figure 3.2.-3. Its purpose is fast access to models that contain several blobs as elements.

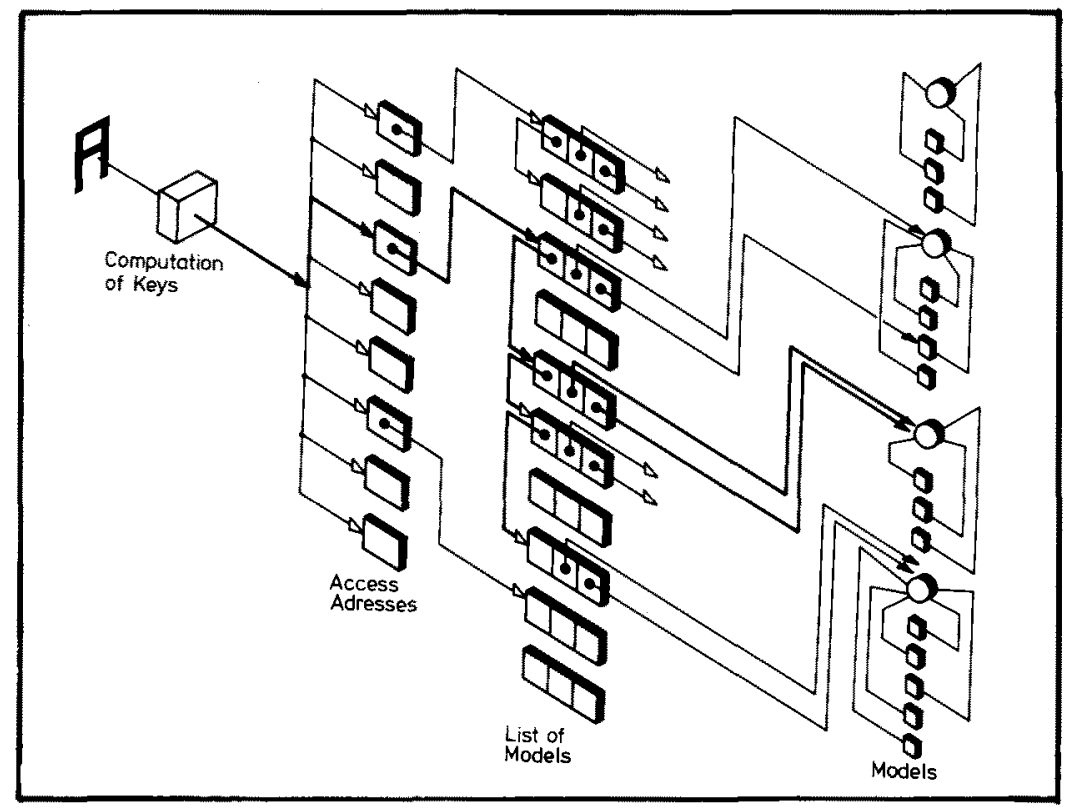

Figure 3.2.-3: The Mode1.Access.Structure

As in the AREA.KEY.TABLE the MODEL.ACCESS.STRUCTURE is accessed by hash coding of area values - in this case we have chosen a linear coding (division by a constant). The MODEL.LIST differs from the AREA.LIST in the fact that it contains two pointers instead of one. While AREA.LIST pointers refer to ST line numbers, the MODEL.LIST pointers reference a model space. The first pointer refers to the model head, the second points to the corresponding blob directly. The corresponding procedures to AREA.SORT and AREA.SEARCH are the procedures: MODEL.SORT and MODEL.ACCESS. The first procedure generates the MODEL.ACCESS. STRUCTURE, the second procedure delivers a list of model and blob addresses for a given area value. In other words, if one has found a blob in the image with a particular area value, MODEL. ACCESS tells in what models -- and where in the models -- such a blob appears. This information is stored in a queue such that candidates can be worked off sequentially. 
The recognition program 'POLAR. CHECK' is based on the assumption that a binary image of a workpiece may well consist of more than one blob. Among those, one blob is said to be the dominant blob (DOM); if there is only one blob it is automatically DOM. In the case of several blobs one should be selected as DOM (in the Teach-In-phase) that is easy to distinguish from the other blobs. All other blobs are called 'satellites' (SATS). The orientation of DOM in the image plane is obtained with the aid of the 'polar.check' which also verifies the features of DoM. This polar. check is an algorithm that scans several circles around the centroid of DoM, determines the intersections of the circles with the contour, and connects these intersections with the centroid. Thus, one obtains a sequence of angles that can be used for recognition and computation of rotational orientation as well (cf. Fig. 3.2.-4).

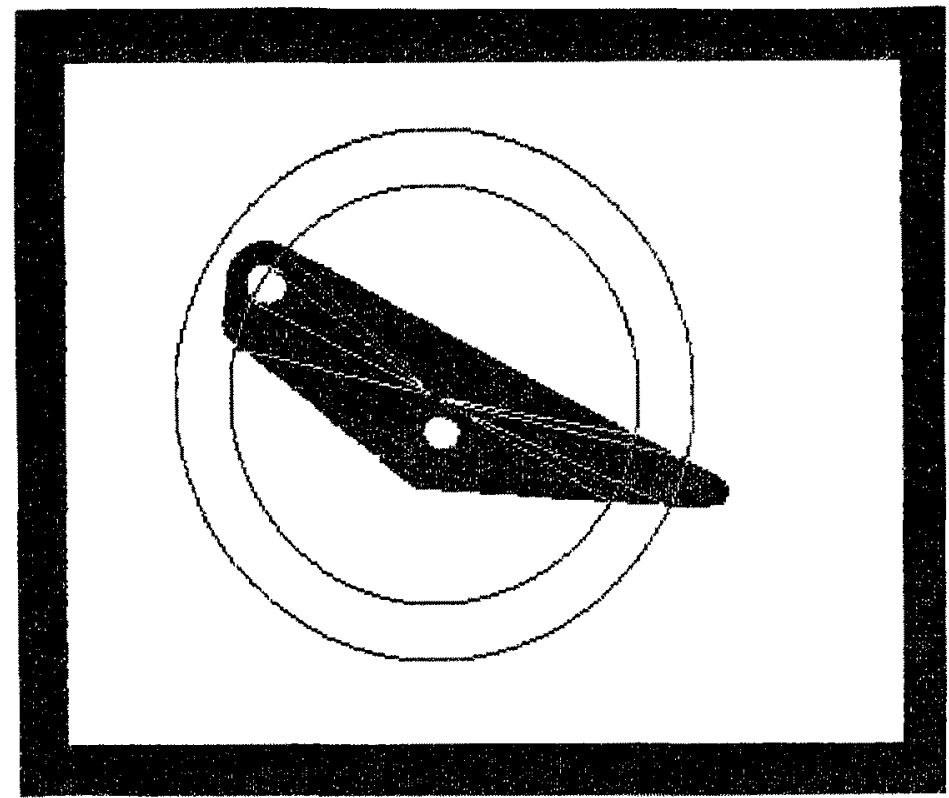

Fig. 3.2.-4: 'Polar.Check' For Workpiece Recognition And Computation of Rotational Orientation 


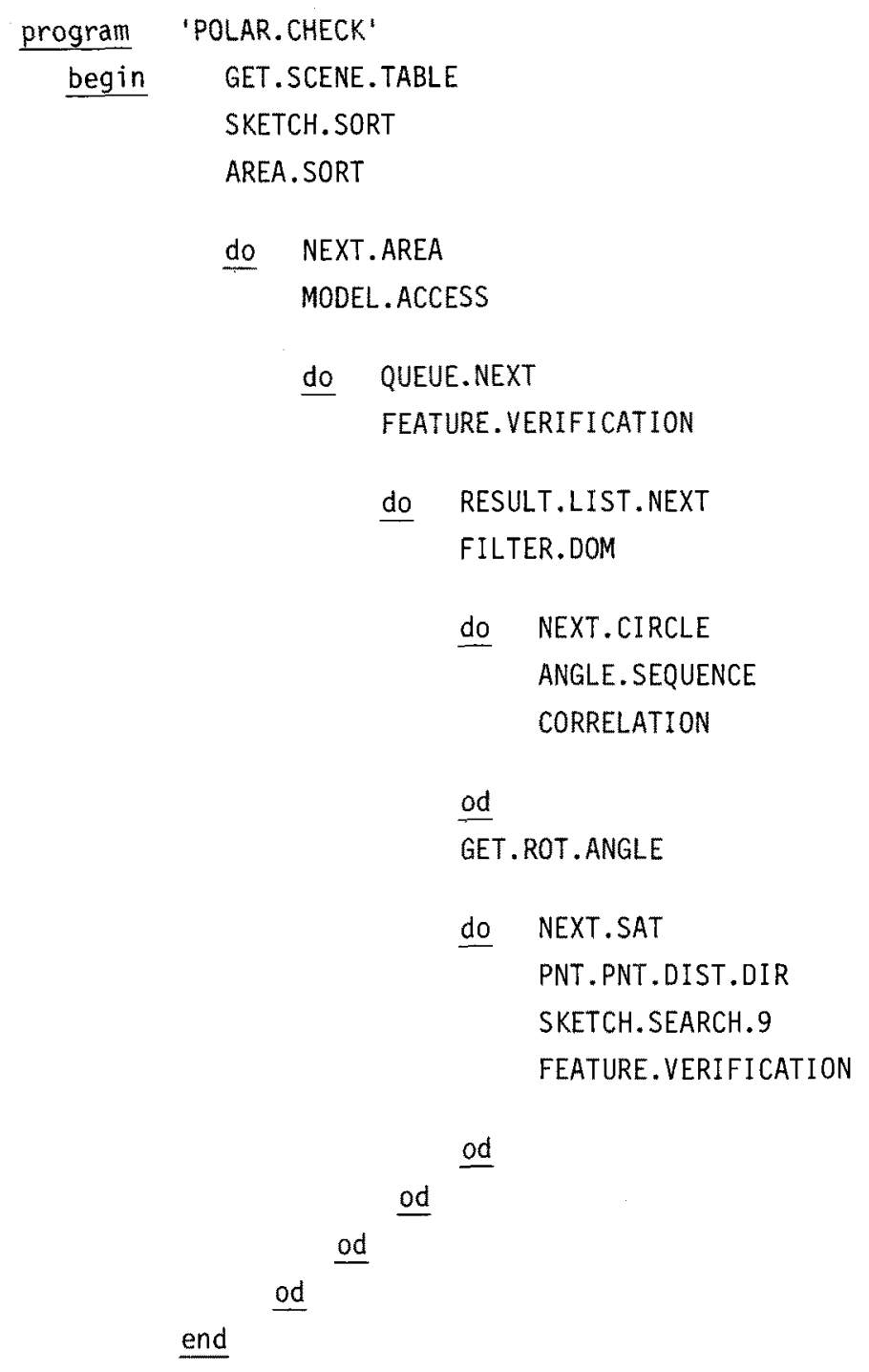

As can be seen from the listing, the program first generates the Scene Table and organizes its data through SKETCH.SORT and AREA.SORT. In the main 10op, blobs are selected one by one whose area values indicate that they might be a DOM. During the Teach-In phase the range of area values of DOMs (amin/amax) is determined. The procedure NEXT.AREA fetches one area value from the AREA. LIST and passes it on to MODEL. ACCESS which generates a queue of model and blob addresses for that area value. QUEUE. NEXT pops one pair of addresses from 
that queue and the model features are compared with the features of the actual blob by the procedure FEATURE. VERIFICATION: In case of a match the corresponding blob is entered into the result list as a hypothetical DOM. Before scanning circles around that DOM it is necessary to separate it from neighbouring blobs. Otherwise the circles would intersect not only the DOM contour but also conours of surrounding blobs (see for instance Fig. 3.2-5A where the triangle shaped DOM in the center has two satellites close by). Therefore the image is transferred from Image Memory 1 to Image Memory 2 in the filtering mode where the labels of the DOM are flagged. As a result one obtains an image that only contains the DOM(Fig. 3.2-5B). Now a circle is drawn around the centroid of the DOM and the intersections of circle and contour are determined. The result is a sequence of angles. In the CORRELATION procedure this sequence is rotated against a stored sequence of the model until both sequences match. If they do not match that blob is rejected as DOM and the next blob is tested. In case of a match the correlation delivers the rotational angle in the image plane. After repetition of the polar.check with other circles the average rotational angle is computed as final result (GET.ROT. ANGLE).

In many cases it is possible to recognize a workpiece with the aid of just one blob; yet in many cases it is feasible to continue with the analysis in the following way. Since location and orientation of the DOM are now known it is easy to predict where the satellites ought to be provided that the model contains distance and direction data from DOM to the SATs. This is indeed given: the model contains for each satellite an entry for: the distance (DIST) from DOM-centroid to SAT-centroid, the direction (DIR) of that connection line (in terms of a counter-clock-wise angle that refers to an initial DOM orientation), and the features of the corresponding blob. 


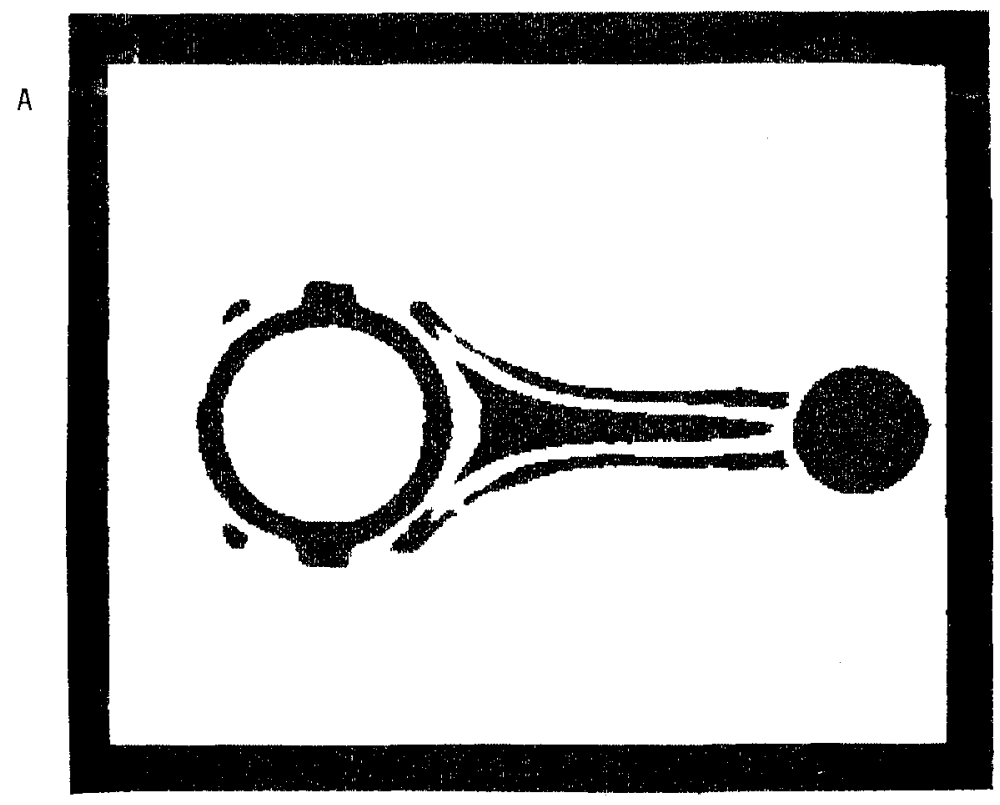

B

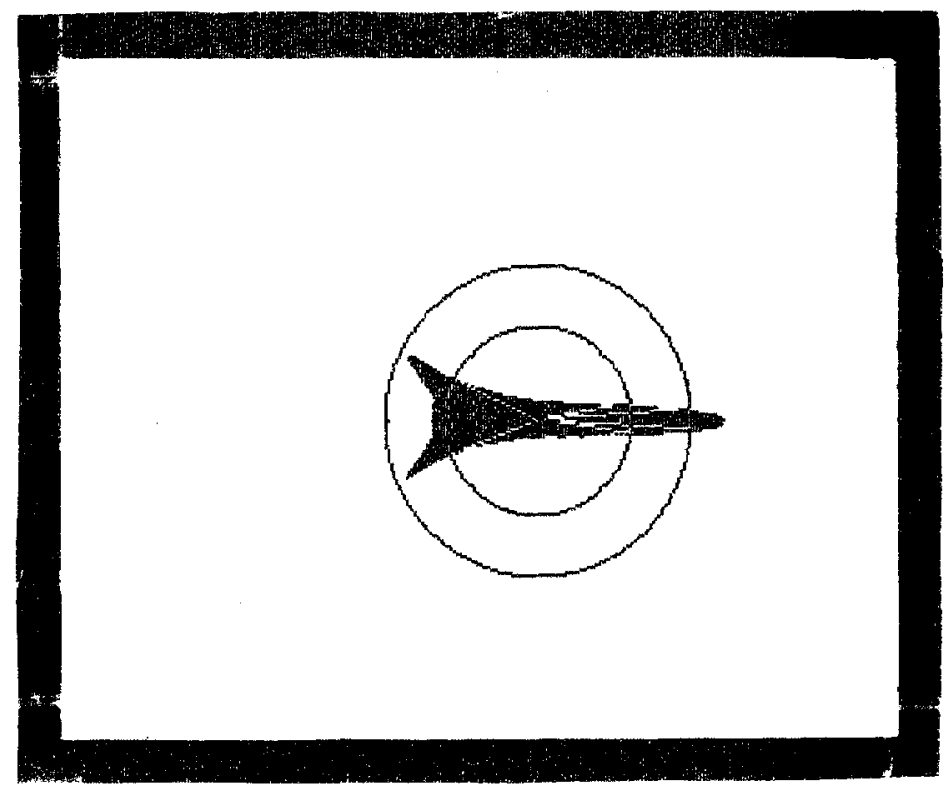

Fig. 3.2.-5: 'Polar. Check' After Filtering the Dominant Blob 
By adding the computed rotational angle of DOM to the initial orientation one computes the actual direction where the satellite must be located. The procedure PNT.PNT.DIST.DIR computes that location and SKETCH.SEARCH. 9 delivers a list of candidates for that SAT. The procedure FEATURE.VERIFICATION selects that blob that matches best. The search for satellites is repeated until all SATs are found. For a model that contains several SATs see the section on 'operator oriented software' (Fig. 3.2.-8). With the aid of such model it is easy to distinguish between workpieces whose dominant blobs are very sinilar but whose satellites look different. This is often the case when stable positions of workpieces occur for similar yaw/pitch/roll coordinates of the workpiece (i.e. by slightly turning the workpiece from one stable position into another one). In such cases the binary images resemble each other strongly except for one or two blobs that can be found with the aid of these models.

The idea to store relational information in the models can be taken one step further by eliminating the polar.check and relying completely on relations for the image analysis. Before we explain the recognition programm a few remarks about model driven search are in order. A relational model actually consists of two separate structures: 1) the relational structure that determines which blobs are connected, and 2) a control structure that determines the order in which these blobs are searched for. In order to avoid explosion of our search space not all blobs are treated in the same way. Rather, one blob serves as "access region" (ACR) of a model, i.e. this blob must be found first before the model driven search can start. In this way the ACR plays a similar role as DOM in the polar.check. In order to establish a local orientation a second blob is needed that is also treated differently tha $\mathrm{n}$ the other satellites: SAT1. The connection of the centroids of ACR and SAT1 defines the orientation in the image plane. All other blobs are attached to the ACR in the same way as in the polar.check. Instead of using blobs, holes may also be used as model elements by simply inverting the polarity of the binary image. Fig. 3.2.-6 shows examples for relational models with blobs and holes (mixed models with blobs and holes are also possible if the image is stored and then read from the Image Memory by inverting it). 


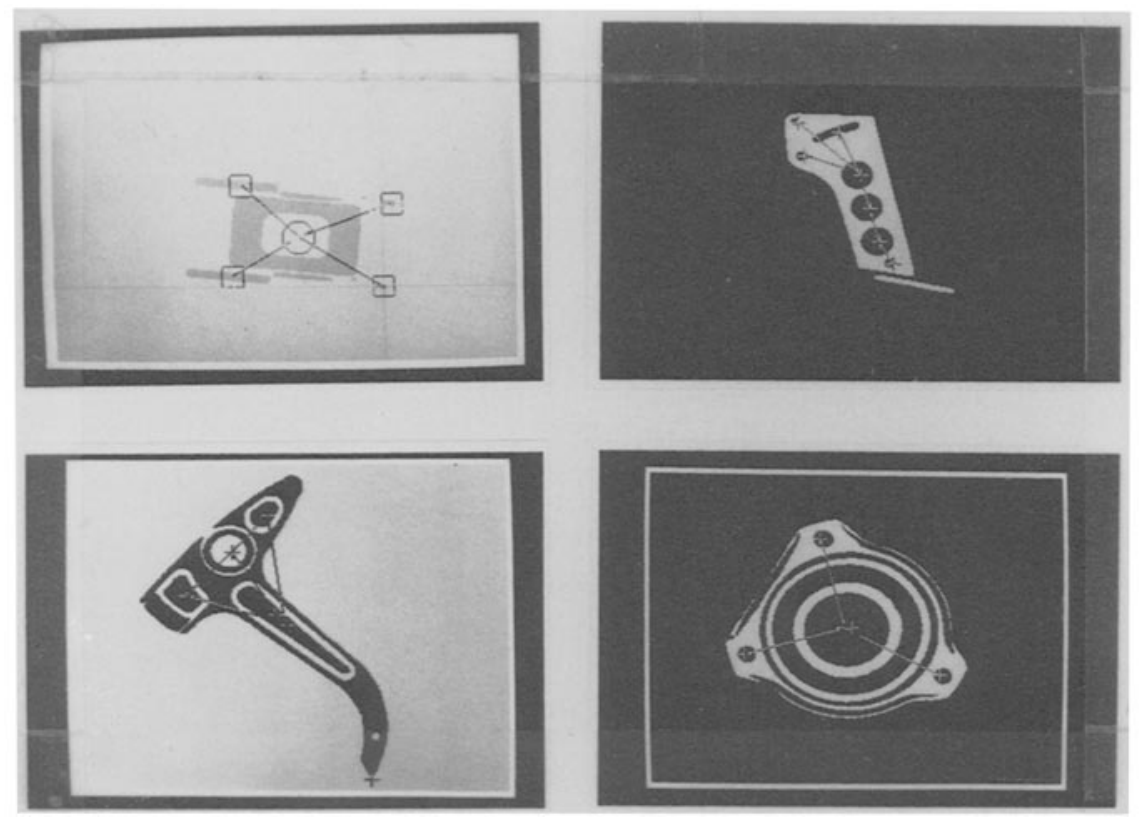

Fig. 3.2.-6: Relational Models Based On Blobs (Left Row) or Holes (Right Row)

The star-like models that we are using right now are rather simple structures but have proven useful in many cases. The investigation of other structures is the topic of on going research.

Let us now give the program in its present version. 
program 'MODEL.SEARCH'

begin GET.SCENE.TABLE

SKETCH.SORT

AREA.SORT

CHECK. TOTAL. AREA

do NEXT.MODEL

GET.MODEL. FEATURES

FEATURE.VERIFICATION

do RESULT.LIST.NEXT

SKETCH.SEARCH.T

FEATURE. VERIFICATION

CHECK.DIST.ACR.SAT1

DIR.ACR.SAT1

$\underline{\text { od }}$

do RESULT.LIST.NEXT

do NEXT.SAT

PNT.PNT.DIST.DIR

SKETCH.SEARCH.9

FEATURE. VERIFICATION

CHECK.DIST.ACR.SAT

CHECK.DIR.ACR.SAT

$\underline{\mathrm{od}}$

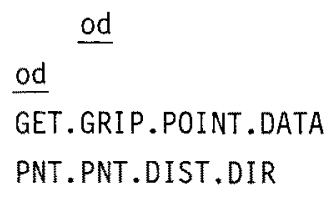

end

As in other programs, the data of the Scene Table are organized previous to the analysis. Before the analysis starts, the total area of the image is checked. If it is too small then another image is read in. Otherwise the analysis starts with the search for an ACR. The features of the next model are passed on to FEATURE. VERIFICATION which delivers a result list that contains likely ACRs and 
their corresponding models.

For each such ACR a search is started in a search window whose size is determined by the distance of SAT1 of that model. This is done by SKETCH.SEARCH.T.BTobs within that window are tested by FEATURE. VERIFICATION for matching features. In order to be sure that one has really found SAT1 the distance between ACR and SAT1 is verified. Finally the direction between ACR and SAT1 is computed (defined as the counter-clock-wise angle of the connection line and a horizontal line through the ACR centroid). The following search for the other satellites is performed as in the polar.check program. Since in 'MODEL. SEARCH' the analysis depends mostly on the spatial relations between the blobs, the exact distances and directions are compared with the mode data after the satellite has been found. This is especially necessary if more than one SAT was found in the search window.

There remains a final step (that is also incorporated in the polar.check program): the location of the workpiece is defined by the centroid coordinates of either DOM or ACR. Yet, most often this is not a point where the gripper of a robot could grasp the object. It is therefore necessary to define a "gripping point" and relate it to the workpiece location. This is easily done: In the model are stored distance and direction of the gripping point from the DOM/ACR location and orientation. The procedure PNT.PNT.DIST.DIR computes with these data the location of the gripping point which is the final result.

\section{OPERATOR ORIENTED SOFTWARE}

The third layer of S.A.M. software is operator oriented and supports the untrained operator during in-site programming. Such programming is necessary to adapt the recognition programs to new workpieces or new inspection tasks. All these software modules are dialogue oriented. Typically, the operator uses a keyboard and a crosshair for programming. With the keyboard he inputs single characters that were proposed to him by a menu on a TV monitor. If the operator needs to input information about the image he simply 'points' with the aid of the crosshair.

A11 recognition programs rely on data that had to be programmed into the system during an instruction phase. It is the purpose of this software layer to support this instruction phase. In the following, we will explain the instruction programs for the three recognition programs that were described in 
the last section. Basically, they all work in the same way. A workpiece is put in front of the camera and the operator points to certain blobs in the binary image while keying in some information about the meaning of those blobs. Since instruction is performed through 'teaching by showing' we call this type of instruction the 'Teach-In' phase.

Before describing these Teach-In programs it may be noted that S.A.M. can also be used as an interactive system for image analysis -- much like commercial systems that are for instance available for microscope image analysis. Interactive image analysis is important for the application-programmer in order to establish parameters for workpiece models, threshold values, etc.. We have therefore implemented a simple interactive mode that allows to read data that the system computes either by hardware or from the Scene Table. It is obvious that this program can be easily extended to more complex tasks.

program 'INTERACTIVE'

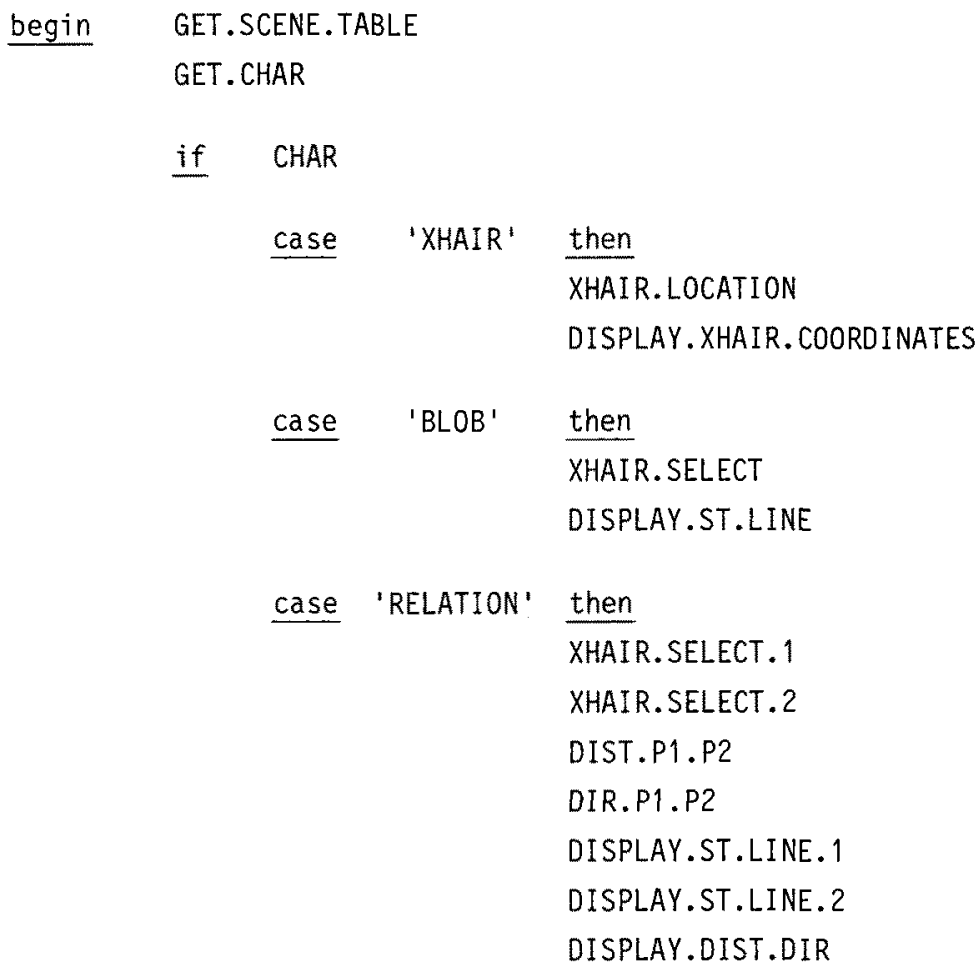


We have not included in the listing the display of the menu which proposes:

$$
X=\text { CROSSHAIR } \quad B=\text { BLOB } \quad R=\text { RELATION. }
$$

Selection of the crosshair allows to read its coordinates from any point in the image. This kind of information may be necessary to design new algorithms. In the 'BLOB' case one marks a blob with the crosshair; the system then displays the corresponding Scene Table line with the extracted features. For the construction of relational models it is important to know distances and directions as well. For this purposes one may select 'RELATION'. Here, the user marks two points or blobs with the crosshair and the system computes distance and direction (from the first point to the second) between the marked points/ blobs. These data are displayed together with the features of the two blobs.

The Teach-In program for the NEAREST.NEIGHBOUR.CLASSIFIER is very simple since we had assumed that each class consists only of one blob. Thus, during Teach-In one must just indicate a particular blob with the crosshair. The corresponding features from the Scene Table are then put into the class list. This way one specifies blob after blob until finished. The input of ' $Q$ ' for 'quit' terminates the program. Fig. 3.2.-7 shows an example of the performance of this program.

program 'NEAREST-NEIGHBOUR-TEACH-IN'

begin GET.SCENE.TABLE

do GET.CHAR

if $C H A R=$ ' $Q$ ' then exit fi

XHAIR.SELECT

PUT. FEATURES

$\underline{\text { od }}$

end

The Teach-In programs for the polar.check and the model search are very much alike. Two particular procedures that are needed in both programs are PUT.SAT and PUT.GRIP.POINT. With PUT.SAT one attaches satellites to either the DOM or the ACR. This is done by pointing to a blob with the crosshair. The system computes the distance and direction and puts these as well as the blob features into the model. 


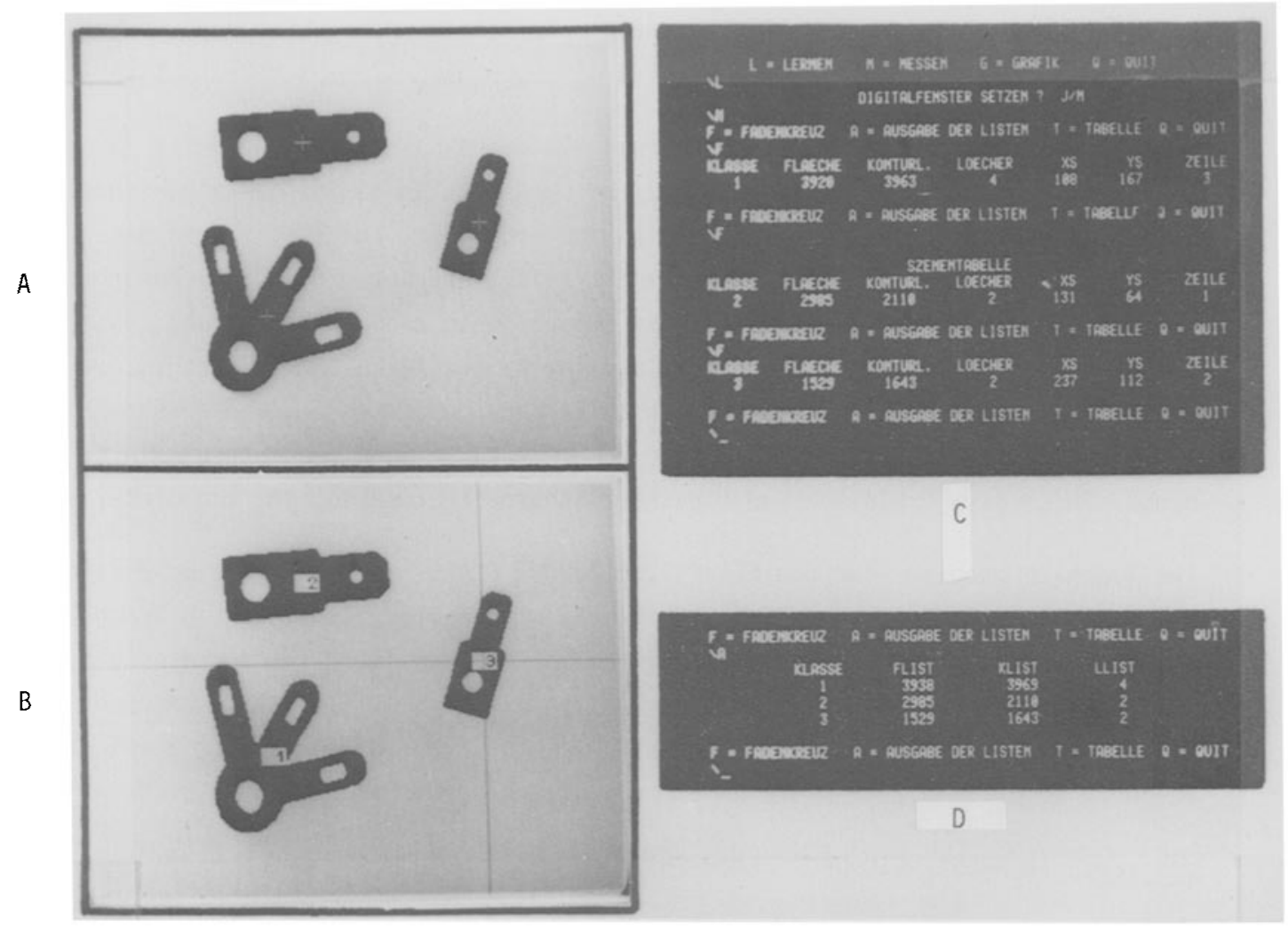

Fig. 3.2.-7: Teach-In Programming with The Crosshair $\begin{array}{ll}\text { A -- Binary Image } & \text { B -- Pointing With Crosshair } \\ \text { C -- Dialogue } & \text { D - Class List }\end{array}$

procedure 'PUT.SAT'

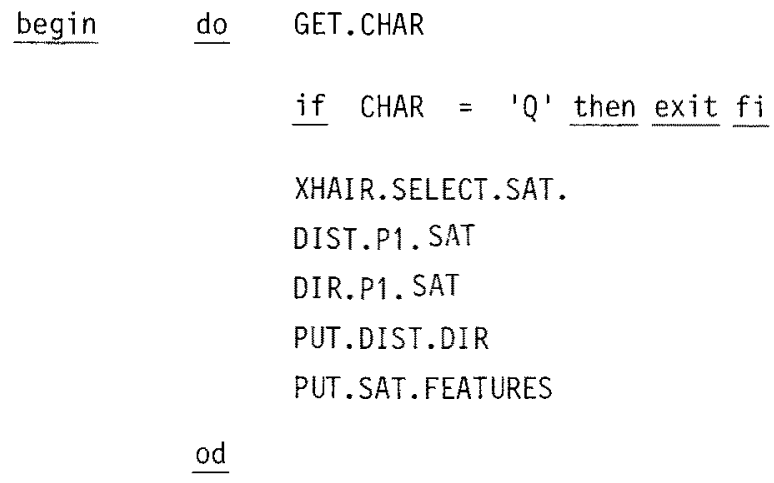


As was already pointed out, the gripping point must not always coincide with the centroid of DOM/ACR. The location of a good gripping point is easily marked with the crosshair. Again, distance and direction to DOM/ACR are computed and put into the model.

procedure 'PUT.GRIP.POINT'

$$
\begin{array}{ll}
\text { begin } & \text { XHAIR.SELECT.GRIP.POINT } \\
\text { DIST.P1.GRIP.POINT } \\
\text { DIR.P1.GRIP.POINT } \\
\text { PUT.DIST.DIR }
\end{array}
$$

end

With these procedures it is easy to understand the two following Teach-In programs. In POLAR. CHECK. TEACH. IN one first selects a DOM whose features are automatically entered into the model. The system then filters DOM from Image Memory1 to Image Memory2. There the user specifies up to four radii. The system scans the corresponding circles, determines the angle sequences, and puts them into the model. Finally, satellites and the gripping point are entered into the model. Fig. 3.2.-8 shows an example.

program 'POLAR. CHECK.TEACH.IN'

$\begin{array}{ll}\text { begin } & \text { GET.SCENE.TABLE } \\ & \text { XHAIR.SELECT.DOM } \\ & \text { PUT.DOM.FEATURES } \\ & \text { FILTER.DOM } \\ & \text { PUT.RADII } \\ \text { CIRCLES } \\ \text { PUT. ANGLE. SEQUENCES } \\ \text { PUT.SATS } \\ \text { PUT.GRIP.POINT }\end{array}$

end 


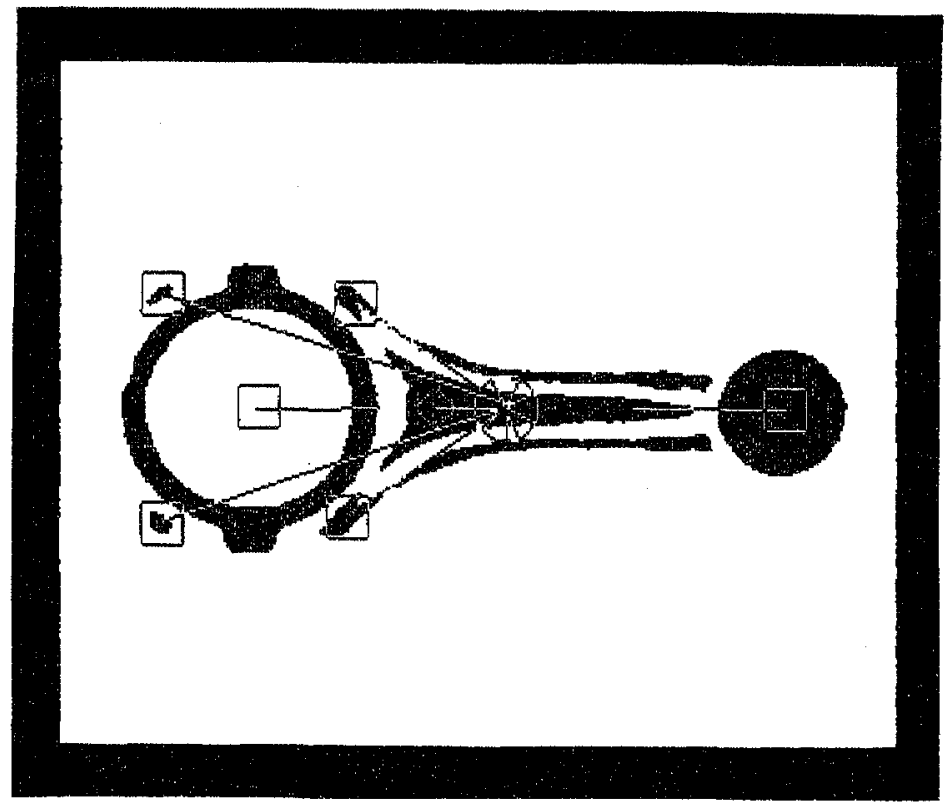

Fig. 3.2.-8: Model For Polar. Check With Satellites

(DOM is represented by a circle; the SATs by squares;

the polar.check of the DOM is demonstrated in Fig. 3.2.-5;

the gripping point coincides with the DOM centroid)

In the MODEL.TEACH. IN the operator marks first the access region ACR and then SAT1 which determines the local orientation. In both cases the system automatically enters the corresponding blob features into the mode? (together with distance and direction data). Then the remaining satellites and the gripping point are entered into the model. Examples for models are shown in Fig. 3.2.-9.

program 'MODEL.TEACH.IN'

begin

GET. SCENE.TABLE

XHAIR.SELECT.ACR

PUT. ACR. FEATURES

XHAIR.SELECT.SAT 1

DIST.ACR.SAT 1

DIR.ACR.SAT 1

PUT.DIST.DIR

PUT.SAT 1.FEATURES

PUT.SATS

end

PUT.GRIP. POINT 
This concludes our description of S.A.M. software. The given programs should be viewed as examples only, since details may vary from application to application. Especially, many details depend on the type of configuration which is used. We will therefore explain the most important types of S.A.M. configurations in the following section.
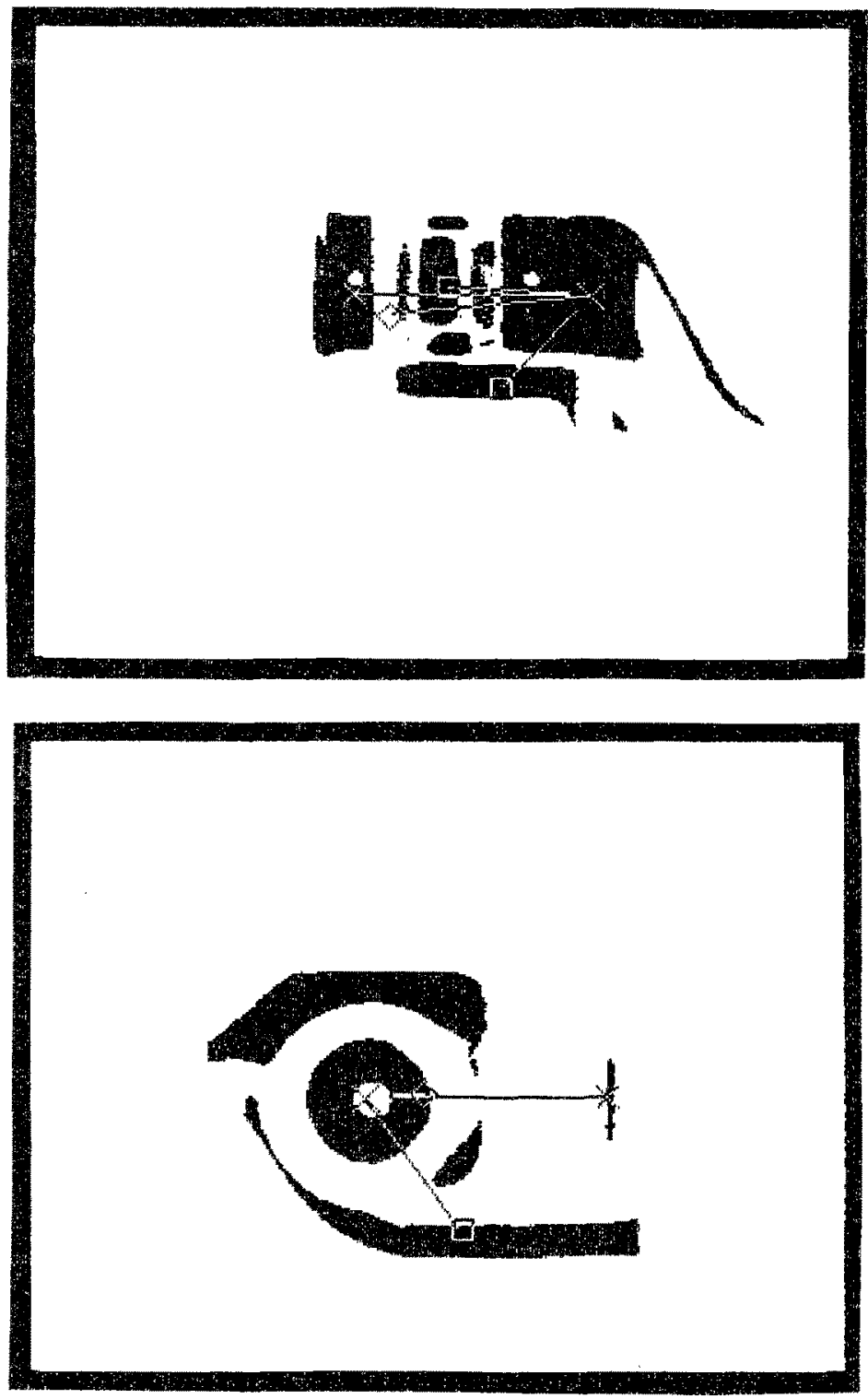

Fig. 3.2.-9: Relational Models

(the diamond represents the gripping point) 


\subsection{S.A.M. CONFIGURATIONS}

S.A.M. hardware was designed such that it can be composed into various configurations that are adapted to the task at hand. It is obvious that all kinds of configurations are possible; here, we want to outline the basic types of configurations. Let us recall that S.A.M. hardware consists of three different groups of modules:

- video circuits

- image memories (IMs), image processing and image analysis processors (IAPs)

- data processors (DPs) and storage.

Video circuits must always be applied; it is possible to synchronize the camera from S.A.M. or vice versa. Furthermore images from two cameras can be read in simultaneousiy into two Image Memories. Other than that, and the choice of an appropriate threshold for binarization, there are not many possibilities to modify the front end of S.A.M. configurations. Configurability is mostly given in the second group of S.A.M. hardware modules, i.e. in the image memories, image processing, and image analysis processors. The most typical configurations are:

- The 'Software Configuration' consists of Image Memories and Data Processing modules. In this configuration everything is done by software. This is certainly the slowest configuration but it is feasible to use it whenever long cycle times are given.

- The 'Image Processing Configuration' consists of two Image Memories, the Image Window Processor (IWP), and Data Processing modules. The purpose of such a configuration is to process images by operations such as shrink, blow, and combinations thereof. Since this configuration includes the Software Configuration it is also possible to perform an image analysis after processing is finished. During image processing, this configuration will typically perform in the ping-pong-processing mode where an image is transferred hence and forth between the two IMs. At each transfer one transformation is performed. Typical operator sequences are: shrink/shrink/ shrink/blow/blow; contourline/blow/blow; blow/blow/shrink. Since each transformation requires only $20 \mathrm{~ms}$, sequences with up to 5 operations can still be executed in about $100 \mathrm{~ms}$. 
- The 'Real-Time Configuration' consists of Image Analysis Processors (IAPs) and Data Processing Units. This type of configuration performs image analysis in real-time, i.e. during the TV scan. After the image scan data are collected into the scene Table. The duration of the generation of the Scene Table depends on the complexity of the scene: the more blobs in the image and the more branches in each blob, the longer the time to generate the Scene Table. Time measurements for typical scenes containing several (4-5) workpieces whose silhouettes decompose into several blobs show that the Scene Table can be generated within 40-80 ms; there are of course scenes where it may take several $100 \mathrm{~ms}$ to construct the Scene Table. Yet, in general the data are organized for further analys is after about $100 \mathrm{~ms}$.

- Combined Configurations consist of mixtures of all other configurations and are likely to be the most important configurations since they are the ones that are really adapted to particular tasks.

Figure 3.3.-1 shows an example for a configuration that might be called the 'maximal' configuration since it provides all operating modes that S.A.M. is now capable of. In particular, this configuration can perform:

- ping pong processing

- real time data extraction

- filtering.

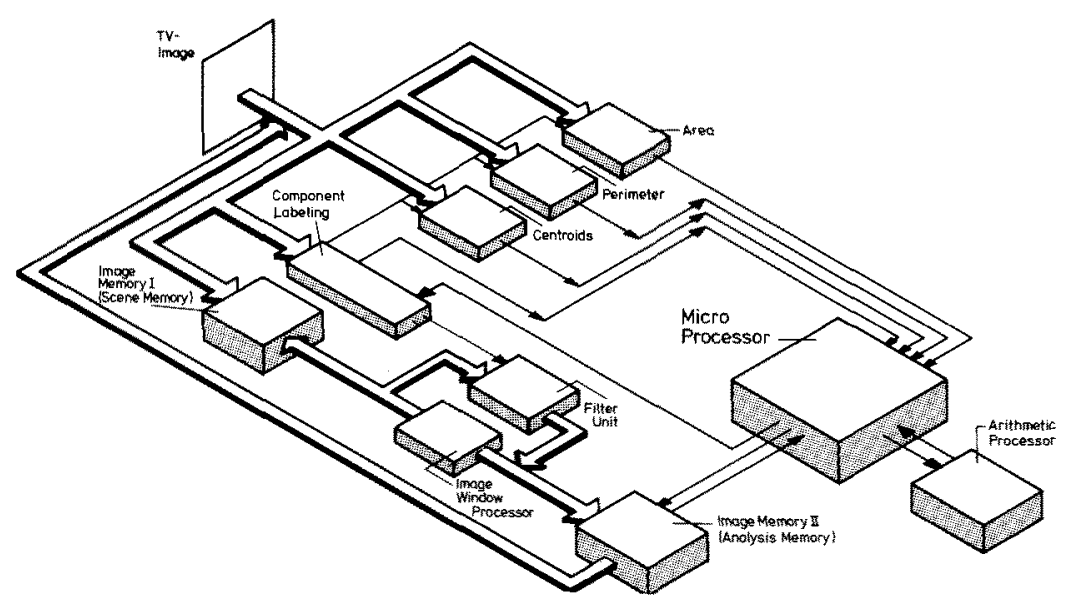

Fig. 3.3.-1: Data Flow In A 'Maximal' Configuration 
Let us follow the flow of data in such a configuration. First the image is read into Image Memory 1. From there it is transferred hence and forth between IM1 and IM2 while the IWP applies some operator to the image (ping pong processing). After $N$ such operations the image is then routed to data extraction. This is done by outputting the image from the actual IM onto the bus. All IAPs extract data from that preprocessed image. These data are then collected by the microprocessor (with the aid of the arithmetic processor). From the scene Table are then selected blobs that are of particular interest. The labels of these blobs are then flagged in the Component Labeling Module (CLM). After flagging, the image is once again transferred from one IM to the other one (the system takes care that this transfer always takes place from IM1 to IM2). During this transfer the CLM is in its filtering mode where all unflagged labels (and their corresponding blobs) are suppressed from the image. As a resuit one obtains a new image that only contains those blobs that were selected previously. Fig.3.3.-2 shows an example for this filtering. In such a filtered image further analysis either by hardware or software can be performed much easier than in the original image.

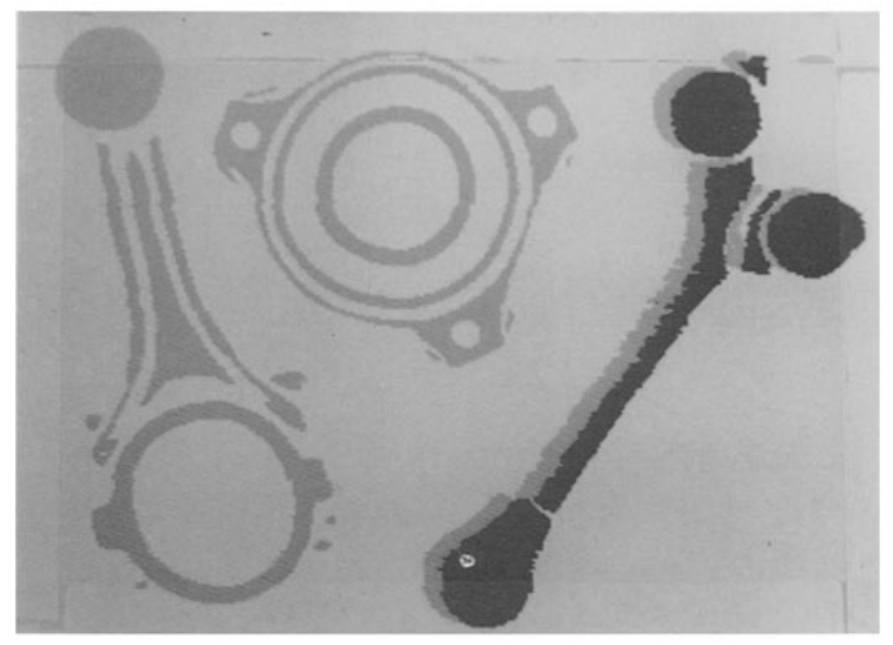

Fig. 3.3.-2: Filtering of Blobs From Image Memory 1 To Image Memory 2 (gray blobs are in IM1, black blobs are in IM2)

It is possible to perform the analys is of rather complex scenes with such a configuration in less than $500 \mathrm{~ms}$. There are other configurations; yet, these examples should suffice to show the processing power of the S.A.M. hardware and software. 


\subsection{THE GRIPPING OF PARTS FROM A RUNNING CONVEYOR BELT}

The gripping of parts from a conveyor belt has found wide-spread interest. Early solutions suffered from the fact that the belt had to be stopped for image acquisition and analysis as well as for part gripping.

We have chosen this task as a first application of a S.A.M. configuration because here a complex analysis must be performed in a very short time. With a visual field of $30 \mathrm{~cm}$ length and a belt running at $30 \mathrm{~cm} / \mathrm{s}$, it is necessary to perform the image analysis in less than $500 \mathrm{~ms}$ so that parts can be 'seen' at least once when passing through the visual field.

In order to avoid motion blur at this high belt speed, very short image acquisition times are necessary. These can be reached with the use of a flashlight. We have designed an infra-red flashlight which has the following advantages:

1) the light frequencies are adapted to the spectral sensitivity of a silicon TV camera;

2) the reflected light can be filtered out in a narrow band, thus effects of the surrounding light are eliminated;

3) the light is invisible to the human eye so that the permanent flashing does not disturb workers at nearby workplaces.

Fig. 3.4-1 and 2 show the experimental set-up that we are using:

- conveyor belt with path measurement

- flashlight

- camera and S.A.M. configuration (with robot interface)

- robot computer and robot

- pallet.

Details about the robot and its control can be found in /STEUSLOFF $180 /$. The movements of the conveyor belt are constantly monitored both by the S.A.M. configuration and the robot computer with the aid of a path measurement. Sensor and robot computer are coupled via the robot interface (cf. section 3.1 ). The task that has to be solved is to grasp parts from the running belt and put them onto a pallet. Workpieces can be positioned on the belt at random but should not overlap. 


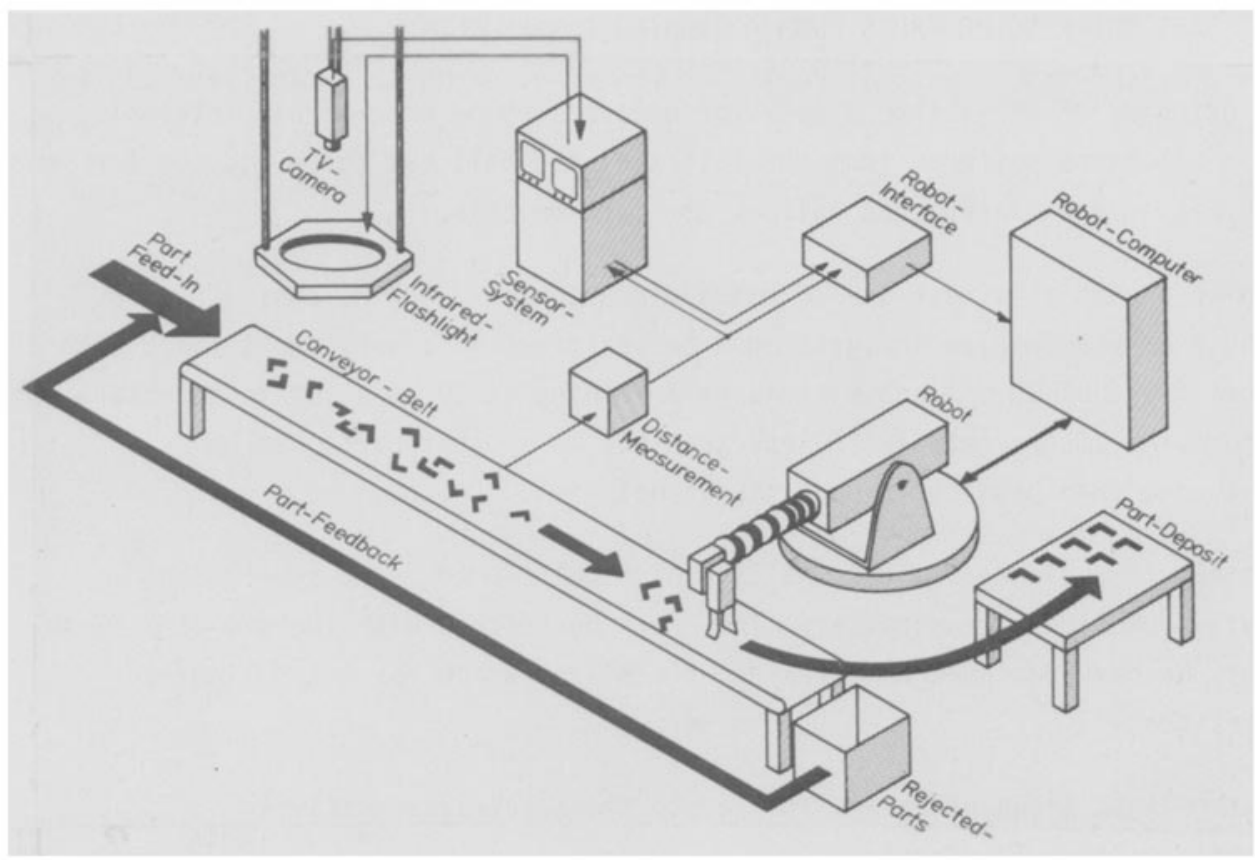

Fig. 3.4.-1: Experimental Set-Up (Diagram)

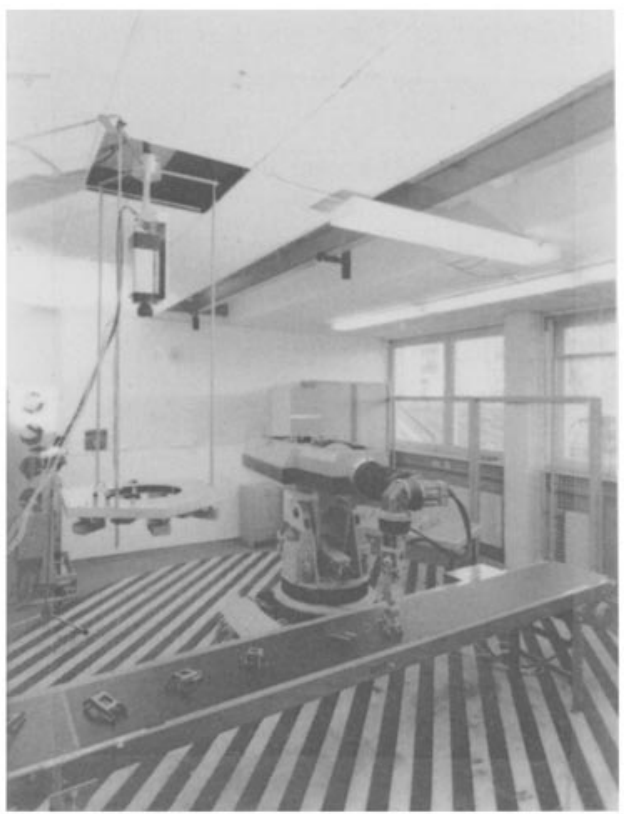

Fig. 3.4.-2:

Experimenta1 Set-Up

(Photography) 
Fig. 3.4.-3 shows a typical scene that the S.A.M. configuration can analyze completely within less than $300 \mathrm{~ms}$. It should be noted here that industrial robots that are available today are not able to grasp fast enough to cope with such high speeds and the multitude of workpieces; for this reason, during the actual handling experiments, there were fewer parts on the belt (as in Fig. $3.4 .-3)$ and the belt was running at a lower speed $(12 \mathrm{~cm} / \mathrm{s})$. Instead of a robot one could also use a computer controlled gate for part sorting.
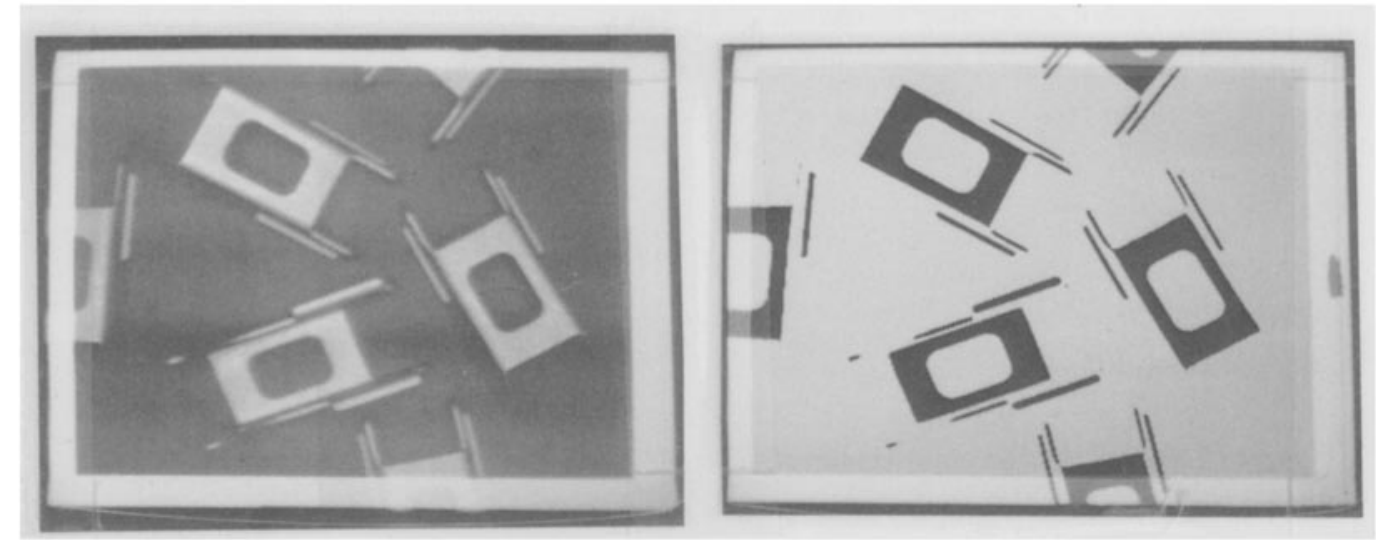

Fig. 3.4.-3: Workpieces On Conveyor Belt

$$
\text { A -- Gray-Level Image B -- Binary Image }
$$

In the handling experiments, the sensor ignites in the beginning flashes contineously and analyzes the image for the appearance of blobs. When the first part enters the image the sensor starts recognition. This will only succeed when all blobs of a workpiece are in the image. Typically it takes $1-2$ images before this happens. At the time of the flash, i.e. the time of image acquisition, an interrupt signal is transmitted to the robot computer which then starts to integrate the path that the belt covers. After the image analysis, the S.A.M. configuration transmits to the robot interface the positional classes of all workpieces in the image together with their locations (of the gripping point) and their rotational angles. Before transmission, these data are converted from pixel coordinates to metric dimensions $(\mathrm{mm})$.

One problem arises from the fact that the same workpiece may appear in the next image again (somewhat further down in the visual field). If care is not taken then the sensor would compute and transmit the data of that workpiece again, and the robot would try to grasp that part a second time. We therefore need a 
'belt description' which contains not only information about workpiece location but which also indicates whether a workpiece has already been 'seen' and transmitted or not. For this reason the S.A.M. configuration integrates the belt path between consecutive images. While reading in a new image the $Y$-coordinates of the belt description are updated by the belt path. By comparison between the belt description and the coordinates of recognized blobs in the new image one can determine which of the blobs were already transmitted to the robot. Thus, information about each workpiece on the belt is transmitted to the robot computer only once. It may be noted that these data have to be transformed into the coordinate system of the robot. This is done in the robot computer which also determines the path of the robot and performs path control. For details see /STEUSLOFF '80/.

\subsection{CONCLUSION}

A prototype S.A.M. configuration ('maximal' version) has been up and running now for two years and we have built six more systems in the mean-time. All configurations have proved to be well suited for the required applications. There are two features that we wish to incorporate into the system in the near future: better thresholding techniques (such as an adaptive threshold) and one more Image Analysis Processor: the computation of moments of inertia.

It has often been argued that binary images are too simple to allow an efficient analys is of complex objects. We do not share this belief. As long as binary images can be produced in a reproducible way they suffice for most practical applications (even though these images don't resemble the images that the human observer sees). The reproducibility can be supported by good illumination engineering. Due to model based analysis techniques it doesn't matter that the silhouette of a workpiece decomposes into several blobs, quite the contrary: relations between those blobs provide enough information about the type of workpiece as well as its orientation. Since in most cases these models can be constructed such that they contain only a minimal number of elements, problems with the explosion of the search space can be avoided.

Our future work will be oriented towards two goals: firstly, we want to implement more (and more complex) operators in the Image Window Processor; 
secondly, we want to extend the structures of the relational models and investigate methods of automatic model generation.

Let us briefly summarize the basic philosophy that has guided us in the design of the system:

1. Whenever possible, mass data must be reduced as much as possible; most often this is done by dedicated hardware which is the core of our system.

2. Complex computations must be avoided and replaced by table look-ups; since such tables tend to be memory consuming we have facilitated such look-ups by providing an extremely wide address space.

3. Data must always be organized such that they can be easily accessed; this is done by sorting of data as well as providing access structures.

These guidelines have led to a system that shows how the analysis of complex binary images can be performed in real-time with reasonable efforts in software and hardware. Due to the modular concept of S.A.M. specific configurations can easily be built that provide economic solutions for practical tasks.

\subsection{ACKNOWLEDGEMENT}

S.A.M. has been developed by E. Enderle, C. Eisenbarth (IBAT-AOP, Essen), J.P. Foith, H. Geisselmann, H. Ringshauser, and G. Zimmermann.

The development was supported by the German Minister of Research and Technology (BMFT), the German National Science Foundation (DFG), and several companies. Responsibility for the content of the work is completely with the autors. 
4. Literature

11/ Abbraham, R.G. Stewart, R.J.S. Shum, L.Y.

"State-0f-The-Art in Adaptable-Programmable Assembly Systems".

International Fluidics Services Ltd. (Pub1.), Kempston, Bedford, UK, 1977.

12/ Agin, G.J.

"An Experimental Vision System for Industrial Applications".

Stanford Res. Lab., Tech. Note 103, Menlo Park, CA, USA, June 1975 .

13/ Agin, G.J.

Duda, R.0.

"SRI Vision Research for Advanced Industrial Automation".

Proc. USA-Japan Computer Conference, Tokyo, August 1975.

14/ Agin, G.J.

"An Experimental Vision System for Industrial Application".

Proc. 5th Int. Symp. on Industrial Robots, Chicago, I11., September 1975.

15/ Agin, G.J. (A)

"Vision Systems for Inspection and for Manipulator Control".

Proc. of the 1977 Joint Automatic Control

Conference,

pp. $132-138$

16/ Agin, G.J.

(B)

"Servoing With Visual Feedback".

Proc. 7th Int. Symp. on Industrial Robots, Tokyo, October 1977, pp. 551-560.

/7/ Agin, G.

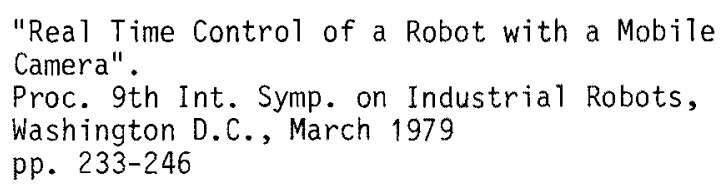

18/ Agrawala, A.K. KuTkarni, A.V.

"A Sequential Approach to the Extraction of Shape Features".

Computer Graphics and Image Processing 6 (1977), pp. $538-557$

19/ Albrecht, M.

Hille, G. Karow, P. Schöne, $H$. Weber, $\mathrm{J}$.

\footnotetext{
"Teileprüung in Automobilbau mittels Fernsehkamera und Prozessrechner".

Proc. INTERKAMA 1977, Syrbe, M. \& Wi11, B. (Eds.), Fachberichte Messen-Steuern-Rege In 1, Springer-Verlag 1977, pp. 107-117.
} 
/10/ Allen, G.R. Juetten, P.G.

111/ Armbruster, K. Martini, P. Nehr, G. Rembold, $U$. Olzmann, $W$.

112/ Ashkar, G.P. Modestino, J.W.

113/ Batchelor, B.G.

/14/ Batchelor, B.G. (B)

/15/ Baird, M.

(A)

/16/ Baird, M.L. (B)

/17/ Baird, M.L.

/18/ Baird, M.
"SPARC - Symbolic Processing Algorithm Research Computer".

Proc. 'Image Understanding Workshop' Nov. 78, pp. $182-190$.

"A Very Fast Vision System for Recognizing Parts and their Location and Orientation".

Proc. 9th Int. Symp. on Industrial Robots, Washington D.C., March 1979, pp. 265-280.

"The Contour Extraction Problem with Biomedical Applications". Computer Graphics \& Image Processing 7 (1978), pp. $331-355$.

"A Preliminary Note on the Automatic Inspection of Male Screw Threads".

Proc. 3rd Int. Conf, on Automated Inspection and Product Control, Nottingham, UK, April 1978, pp. 139-176.

"SUSIE: A Prototyping System for Automatic Visual Inspection".

4th Int. Conf. on Automated Inspection and Product, Chicago, I11, November 1978, pp. 49-80.

"An Application of Computer Vision to Automated IC Chip Manufacture".

Proc. 3rd Int. Joint Conf. on Pattern Recognition Coronado, CA, November 1976, pp. 3-7.

"Sequential Image Enhancement Technique for Locating Automotive Parts on Conveyor Belts". Gerneral Motors Res. Lab. Pub1. GMR-2293, CS Dept., Warren, MI, USA, Nov. 1976.

"Image Segmentation Technique for Locating Automotive Parts on Belt Convevors". Proc. Int. Joint Conf. on Artificial Intelligence, Tokyo, Japan, Aug. 1977, pp. 694-695.

"SIGHT-I: A Computer Vision System for Automated IC Chip Manufacture".

IEEE Trans. Systems, Man \& Cybernetics SMC -8

(1978) 2, pp. 133-139.

119/ Barrow, H.G.

Popplestone, R.J. "Relational Descriptions in Picture Processing". in: B. Meltzer \& Michie, D. (Eds.): 'Machine Intelligence $6^{\prime}$, University Press, Edinburah, 1971 pp. 377-396. 
120/ Barrow, H.G. Ambler, A.P. Burstal1, R.M.

121/ Birk, J. Kelley, R.B. et.al.

122/ Birk, J. Kelley, R. Chen, N. (A)

123/ Birk, J. Kelley, R. et al.

(B)

124/ Birk, J. Kelley, R. et al.

/25/ Bjorklund, C.M.

/26/ Bolles, R.C. (A)

Bolles, R.C. (B)

/28/ Bretschi, J.
"Some Techniques for Recognizing Structures in Pictures".

in: S. Watanabe (Ed.), 'Frontiers of Pattern Recognition', Academic Press, N.Y., 1971, pp. 1-32

"Orientation of Workpieces by Robots Using the Triangle Method".

SME Tech Paper MR 76-612

Univ. Rhode Island, EE Dept.

Kingston, RI, USA, 1976

"Visually Estimating Workpiece Pose in a Robot Hand Using the Feature Points Method". Proc. IEEE Conf. on Decision \& Control, San Diego, CA, January 1979, pp. $A 1-1-A 1-6$

"General Methods to Enable Robots with Vision to Acquire, Orient, and Transport Workpieces". 5 th report, EE Dept., Univ. of Rhode Island, Kingston, RI, USA,

Aug. 1979

"General Methods to Enable Robots with Vision to Acquire, Orient and Transport Workpieces". 6th report

EE Dept., Univ. of Rhode Island, Kingston, RI, USA, Aug. 1980.

"Syntactic Analysis \& Description of Stroke-Based Shapes".

Proc. IEEE Conf. Pattern Recognition and Image Processing, Troy, NY, USA, June 1977, pp. 198 202.

"Robust Feature Matching through Maximal Cliques". SPIE Vol 182 'Imaging Applications for Automated Industrial Inspection \& Assembly ; Washington, D.C., USA, April 1979, pp. 140 - 149.

"Part Acquisition Using the SRI Vision Module". Proc. 3rd IEEE Computer Software \& Applications Conference COMPSAC-79, Chicago, I11., November 79 , pp. 872-877

"A Microprocessor Controlled Visual Sensor for Industrial Robots".

The Industrial Robot $\underline{3}$ (1976) 4, pp. 167-172. 
129/ Brook, R.A.

Pur 11, D.J.

Jones, G.H.

Lewis, D.0.

130/ Burow, M. Wahl, F.

/31/ Callen, J.E. Anderson, P.N.

/32/ Claridge, J.F. Purll, D.J.

/33/ Colding, B.

Colwe 11, L.V.

Smith, D.N.

134/ Cronshaw, A.J. Heginbotham, W.B. Pugh, A.

/35/ Cronshaw, A.J. Heginbotham, W.B. Pugh, A.

/36/ Davis, L.S.

/37/ De Coulon, D. Kammenos, $P$.

/38/ Dessimoz, J.-D.
"Practical Experience of Image Processing in OnLine Industrial Inspection Applications". SPIE Proc. Vol. 130, Automation and Inspection Applications of Image Processing Techniques, London, Sept. 1977, pp. 84-97.

"Eine verbesserte Version des Kantendetektionsverfahrens nach Mero/Vassy".

'Angewandte Szenenanalyse', J.P. Foith (Ed.), Informatik-Fachbericht 20, Springer-Verlag, Heidelberg, 1979, pp. 36-42.

"Checking Labeled Bottles Electro-0ptically". Electro-Optical Systems Designs, July 1975, pp. 44-46.

"Automatic Inspection \& Gauging Using Solid-Sate Image Scanners".

3rd Int. Conf. on Automated Inspection and Product Control, Nottingham, UK, April 1978, pp. 31-41.

"Delphi Forecasts of Manufacturing Technology". International Fluidics Services (Publ.) Kempston, Bedford, UK, 1979

"Software Techniques for an Optically Tooled Bowl Feeder".

3rd Int. Conf. on Trends in On-Line Computer Control Systems, Univ. of Sheffield, UK, March 1979, pp. 145-150.

"A Practical Vision System for use with Bow] Feeders".

Proc. ist int. Conf. on Assembly Automation, Brighton, UK, March 1980, pp. 265-274.

"A Survey of Edge Detection Techniques". Computer Graphics and Image Processing 4 (1975) pp. $248-270$.

"Polar Coding of Planar objects in Industrial Robot Vision". Neue Technik (NT) (1977) 10, pp. 663 - 671

"Identification et Localisation Visuelle D'objets Multiples Par Poursuite de Contour et Codage de Courbure". Compte-Rendus Journées de Microtechnique, EPFLausanne, Suisse, Sept. 1978 
/39/ Dessimoz, J.D. Kunt, $M$.

Zurcher, J.M. Granlund, G.H.

140/ Dodd, G.G. Rossol, L.

141/ Duda, R. Hart, $P$.

142/ Duff, M.J.B.

/43/ Ehrich, R.W.

"Detection of Global Edges in Textured Images". IEEE. Trans. Comp. C-26 (1977) 6, pp.289-603

/44/ Ejiri, M. Uno, T.

Mese, M. Ikeda, S.

/45/ Enderle, E.

146/ Eskenazi, R. Wilf, $\mathrm{J}$.

"A Process for Detecting Defects in Complicated Patterns".

Computer Graphics and Image Processing (1973) 2, pp. 326-339.

"Ein Baukastensystem fü Bildsensoren zur Sichtprüfung und Prozeßsteuerung".

PDV-Bericht "INTERKAMA'80", W. Hofmann, (Ed.), Kernforschungszentrum Karlsruhe, August 1980, Kfk-PDV, pp. 358 - 365 .

"Low Level Processing for Rea1-Time Image Analysis". Proc. IEEE Comp. Soc. 3rd Int. Comp. - Software \& Applications Conference (COMPSAC 179), Nov. 79, Chicago, I11, USA, pp. $340-343$.

147/ Eversole, W.L.

Mayer, D.J.

Frazee, F.B.

Cheek, Jr.J.F.
"Investigation of VLSI Technologies for Image Pro- cessing".
Proc. 'Image Understanding Workshop',
Palo A1to, CA, USA, Apri1 1979, pp. 159 - 163.

/48/ Feng, H.F.

Pavlidis, T.

"Decomposition of Polygons into Simpler Components:

Feature Generation for Syntactic Pattern Recognition".

IEEE Trans. Comp. C-24 (1975) 6, pp. 636 - 650. 
149/ Flöscher, R. Partmann, $T$.

/50/ Foith, J.P.

/51/ Foith, J.P. Geisselmann, H. Lübbert, $\mathrm{U}$. Ringshauser, $H$.

/52/ Foith, J.P.

153/ Foith, J.P. Eisenbarth, C. Enderle, $E$. Geisselmann, $H$. Ringshauser, $\mathrm{H}$. Zimmermann, G.

154/ Frei, W. Chen, Ch.-Ch.

155/ Fries, R.W. Modestino, J.W.

/56/ Geisselmann, H.
"Sensorsystem zum automatischen Aussortieren fehlerhafter kleinteile".

Mitteilungen aus dem Fraunhofer-Institut für Informations- und Datenverarbeitung (IITB), FhG-Berichte 2-80, Karlsruhe 1980, pp. $23-25$

"Lage-Erkennung von beliebig orientierten Werkstuicken aus der Form ihrer Silhouetten".

Proc. 8th Int. Symposium on Industrial Robots, Böblingen, W.-Germany, May/June 1978, pp. 584-599.

"A Modular System for Digital Imaging Sensors for Industrial Vision."

Proc. 3rd CISM-IFToMM Symposium in Theory and

Practice of Robots and Manipulators, Udine, Italy, Sept. 1978, Elsevier, Amsterdam, 1980,pp. 399-422

"A TV-Sensor for Top-Lighting and Multiple Part Analysis."

Proc. 2nd IFAC/IFIP Symposium on Information Control Problems in Manufacturing Technology, Stuttgart, Oct. 1979, U. REMBOLD (Ed.), Pergamon Press, Oxford, 1979, pp. 229-234.

"Optischer Sensor für Erkennung von Werkstücken auf dem laufenden Band - realisiert mit einem modularen System."

in: H. Steusloff (Ed.): "Wege zu sehr fortgeschrittenen Handhabungssystemen", Messen - Steuern Regeln Band 4, Springer-Verlag, Berlin 1980, pp. $135-155$.

"Fast Boundary Detection: A reneralization and A New Algorithm".

IEEE Trans. on Comp. C-26 (1977) 10, pp. 988-998

"An Empirical Study of Selected Approaches to the Detection of Edges in Noisy Digitized Images".

Proc. IEEE Conf. on Patt. Rec. \& Image Processing, Troy,N.Y. USA, June 1977, pp. $225-230$

"Griff in die kiste durch Vereinzelung und optische Erkennung."

in: H. Steusloff (Ed.): 'Wege zu sehr fortgeschrittenen Handhabungssystemen', Fachberichte Messen-Steuern-Regein Band 4, Springer-Verlag, Berlin 1980, pp. 156-165 
/57/ Giralt, G. Ghallab, M. Stuck, $F$.

158/ GTeason, G.J. Agin, G.J.

/59/ Goto, N.

Kondo, T. Ichikawa, K. Kanemoto, $M$.

/60/ Haralick, R.M. Shapiro, L.G.

161/ Hasegawa, $\mathrm{K}$. Masuda, R.

163/ Hill, J.W. Sword, A.J. et al.
164/ Holland, S.W.

"Object Identification and Sorting with an Optimal Sequential Pattern Recognition Method". Proc. 9th Int. Symp. on Industrial Robots, Washington, D.C., USA, March 1979 , pp. $379-389$

"A Modular Vision System for Sensor-Controlled Manipulation and Inspection."

Proc. 9th Int. Symp. on Industrial Robots Washington D.C., March 1979, pp. $57-70$.

"An Automatic Inspection System for Mask Patterns".

Proc. 4th Int. Joint Conf. on Pattern

Recognition, Kyoto, 1978

pp. $970-974$

"Decomposition of Polygonal Shapes by

Clustering".

Proc. IEEE Conf. Pattern Recognition and

Image Processing ' 77 ,

Troy, N.Y., USA, June 1977,

pp. $183-190$

"On Visual Signal Processing for Industrial

Robot".

Proc. 7 th Int. Symposium on Industrial Robots,

Tokyo, Japan, Oct.'1977,

pp. 543-550

162/ Heginbotham, W. B. "The Nottingham 'Sirch' Assembly Robot".

Proc. 1st Conf. on Industrial Robots

Nottingham, UK; 1973

pp. $129-142$

"Programmable Part Presenter Based on Computer Vision and Controlled Tumbling".

Proc. 10th Int. Symp. on Industrial Robots, Milan, Italy, March 1980, pp. 129-140

"A Programmable Computer Vision System Based on Spatial Relationships".

General Motors Res. Lab. Publication GMR-2078 CS Dept., Warren, MI, USA, Feb. 1976 
165/ Holland, S.W.

Rossol, L.

Ward, M.R.

166/ Hsieh, Y.Y. Fu, K.S.

167/ Hueckel, M.H.

168/ Iannino, A. Shapiro, S.D.

/69/ Jarvis, J.F. (A)

170/ Jarvis, J.F. (B)

171/ Kamin, G.

172/ Karg, R.

173/ Karg, R. Lanz, O.E.
"CONSIGHT-I: A Vision Controlled Robot System for Transferring Parts from Belt Conveyors". in: 'Computer Vision and Sensor-Based Robots', G.G. Dodd \& L. Rossol (Eds), Plenum Press, N.Y., 1979, pp. $81-97$

"A Method for Automatic IC Chip Alignment and Wire Bonding".

Proc. IEEE Conf. on Pattern Recognition and Image Processing, Chicago I11., August 1979, pp. 101-108

"An Operator which Locates Edges in Digitized Pictures".

Journal of the ACM 18 (1971) 1, pp. 113-125.

"A Survey of the Hough Transform and its Extensions for Curve Detection". Proc. IEEE Conf. on Patt. Rec. \& Image Processing, Chicago, I11, June 1978, pp. $32-3 c$

"A Method for Automating the Visual Inspection of Printed Wiring Boards".

IEEE Trans. Pami-2 (1980) 1, pp. 77-82.

"Visual Inspection Automation"

IEEE Computer May 1980, pp. 32-38

"Der Geometrie Computer".

rme 40 (1974) 3, pp. 105 - 109

"A Flexible Opto-Electronic Sensor".

Proc. 8th Int. Symp. on Industrial Robots, Stuttgart, W.-Germany, May/June 1978, pp. 218-29

"Experimental Results with a Versatile Optoelectronic Sensor in Industrial Applications".

Proc. 9th Int. Symp. on Industrial Robots Washington D.C. , March 1979, pp. 247-264.

"A Transistor Wire-Bonding System Utilizing Multiple Local Pattern Matching Techniques." IEEE Trans. on System, Man, and Cybernetics SMC-6 (1976) 8, pp. 562-570 
175/ Kashioka, S. Takeda, S. Shima, Y. Uno, $T$. Hamada, $T$.

/76/ Kelley, R.B. Birk, J. Wilson, $L$

/77/ Kelley, R.B. Birk, J. Martins, $\mathrm{H}$. Tella, R.

178/ Korn, A.

/79/ Kruse, B.

/80/ Levialdi, S.

181/ Löffler, H. Jäger, J.

182/ Martelli, A.

/83/ Martini, P. Nehr, G.
"An Approach to the Integrated Intelligent Robot with Multiple Sensory Feedback:

Visual Recognition Techniques".

Proc. of 7 th Int. Symp. on Industrial Robots, Tokyo, October 1977, pp. 531-538

"Algorithms to Visually Acquire Workpieces". Proc. 7 th Int. Symp. on Industrial Robots, Tokyo, Japan, Oct. 1977 , pp. 497-506

"A Robot System which Feeds Workpieces Directly from Bins into Machines". Proc. 9th Int. Symp. on Industrial Robots, Washington D.C., March 1979, pp. 339-355

"Segmentierung und Erkennung eines objektes in natürlicher Umgebung".

in: E. Triend1. (ED.): 'Bildverarbeitung und Mustererkennung ', DAGM-Symposium 0ct. 78, Informatik-Fachberichte Band 17, Springer Verlag, Berlin, 1978, pp. 265-274

"A Parallel Picture Processing Machine". IEEE Trans. Comp. C-22 (1973) 12 , pp. $1075-1087$

"Finding the Edge". Proc. NATO Advanced Study Institute on Digital Image Processing and Analys is, June 23 - July 4, 1980, Bonas, France, pub]. by INRIA, Le Chesnay, pp. $167-208$

"Meßverfahren der Bildanalyse zur Fertigungskontrolle feinmechanischer Präzisionsteile oder elektronischer Bauelemente". messen + prïfen/automatik, Oct. 79 , pp. $755-758$

"Edge Detection Using Heuristic Search Methods". Computer Graphics \& Image Processing 1 (1972) pp. 169-182.

"Recognition of Angular Orientation of objects with the Help of Optical Sensors". The Industrial Robot (1979) June, pp. 62-69. 
/84/ McGhie, D.

Hi11, J.W.

185/ McKee, J.W.

Aggarwal, J.K.

186/ Mero, L.

Vassy, Z.

/87/ Milgram, D.L.

(A)

Milgram, D.L.

(B)

189/ Milgram, D. Herman, $M$.

/90/ Montanari, U.

191/ Mori, K. Kidode, M. Shinoda, H. et al.

192/ Mundy, J.L. Joynson, R.E.

193/ Nakagawa, Y. Rosenfeld, A.
"Vision Controlled Subassembly Station". Society of Manufacturing Engineers (SME) Paper No. MS78-685, 1978

"Computer Recognition of Partial Views of Curved Objects".

IEEE Trans. Comp. C-26 (19-7) 8, pp. $790-800$

"A Simplified and Fast Version of the Hueckel operator for Finding Optimal Edges in Pictures". Proc. IJCAI '75, Tbilisi, USSR, 1975

"Region Extraction Using Convergent Evidence". L.S. Baumann (Ed.), Proc. 'Image Understanding Workshop', Science Applications, Inc.

Arlington, VA, Apri] 1977, pp. 58 - 64

"Progress Report on Segmentation Using Convergent Evidence".

L.S. Baumann (Ed.), Proc. 'Image Understanding Workshop', Science Applications, Inc., Arlington VA, 0ct. 1977, pp. 104-108

"Clustering Edge Values for Threshold Selection". Computer Graphics and Image Processing 10 (1979), pp. 272-280

"On the Optimal Detection of Curves in Noisy Pictures".

Communications of the ACM 14 (1971), pp. 335-345.

"Design of Local Parallel Pattern Processor for Image Processing".

Proc. AFIPS, Vol 47, June 1978, pp. 1025-1031.

\footnotetext{
"Automatic Visual Inspection Using Syntactic Analysis".

Proc. IEEE Conf. on Pattern Recognition and Image Processing, Troy, N.Y., June 1977, pp. 144-147.
}

"Some Experiments on Variable Thresholding". CS Report TR 626, Univ. of Maryland, College Park, MD, January 1978 
194/ Nakagawa, Y. Rosenfeld, A.

195/ Nakamura, K. Edamatsu, $K$. Sano, $Y$.

196/ Nawrath, R.

197/ Nevatia, R. Babu, K.R.

198/ Nitta, Y.

199/ Nudd, G.R. Nygard, P.A. Erickson, J.L.

/100/ Nudd, G.R. Nygard, P.A. Fouse, S.D. Nussmejer, T.A.

/101/ O'Gorman, F.

1102/ Ohlander, R. Price, $K$. Reddy, D.R.

/103/ 01sztyn, J.T.

Rossol, L Dewar, R. Lewis, N.R.
"A Note on Polygonal and Elliptical Approximation of Mechanical Parts".

Pattern Recognition 11 (1979), pp. 133-142.

"Automated Pattern Inspection Based on 'Boundary Length Comparison Method'".

Proc. 4th Int. Joint Conf. on Pattern Recognition Kyoto, 1978.

"LEITZ-T.A.S., neue Möglichkeiten der Bildanalyse".

LEITZ-Mitteilungen Wiss.- $u$. Techn, Band VII (1979) 6, Wetzlar, pp. 168-173

"Linear Feature Extraction and Description". Proc. 6th Int. Joint Conf. on Artificial Intelligence, Tokyo, Auf. 1979, pp. 639-641

"Visual Identification and Sorting with TVCamera Applied to Automated Inspection Apparatus".

Proc. 10th Int. Symp. on Industrial Robots Milan,Italy, March 1980, pp. 141-152

"Image Processing Techniques Using ChargeTransfer Devices".

Proc. 'Image Understanding Workshop'. Palo A1to, CA.USA, Oct. 1977, pp. $1-6$

"Implementation of Advanced Real-Time Image Understanding Algorithms".

Proc. 'Image Understanding Workshop', Palo Alto, CA. USA, April 1979, pp. 151-157

"Edge Detection Using Walsh Functions". Artificial Intelligence 10 (1978), pp. 215-223.

"Picture Segmentation Using a Recursive Region Splitting Method".

Computer Graphics and Image Processing 8 (1978), pp. 313 - 333 .

"An Application of Computer Vision to a Simulated Assembly Task".

Proc. 1st Int. Joint Conf. on Pattern

Recognition, Washington D.C., Oct./Nov. 1973, pp. 505-513 
/104/ Panda, D.P. Rosenfeld, A.

1105/ Pavlidis, T.

/106/ Pavlidis, T.

/107/ Pavlidis, T.

/108/ Perkins, W.A.

/109/ Perkins, W.A.

/110/ Perkins, W.A.

/111/ Prager, J.M.

/112/ Prewitt, J.M.S.
"Image Segmentation by Pixel Classification in (Gray-Level, Edge Value) Space".

IEEE Trans. Comp. C-27 (1978) 9, pp. 875-879

"Structural Pattern Recognition:

Primitives and Juxtaposition Relations". in: S. Watanabe (ed.) "Frontiers of Pattern Recognition, Academic Press, N.Y., 1972 pp. $421-451$

"Structural Pattern Recognition". Springer Verlag, Berlin, 1977.

"A Review of Algorithms for Shape Analysis". Computer Graphics and Image Processing 7 (1978) pp. 243-258

"Mode1-Based Vision System for Scenes Containing Multiple Parts".

Proc. Int. Joint Conf. on Artificial Intelligence, Tokyo, Japan, Aug. 1977, pp. $678-684$

"Computer Vision Classification of Automotive Control Arm Bushings".

Proc. IEEE 3rd Int. Computer Software \& Applications Conference COMPSAC 79, Chicago, I11., November 1979, pp. 344-349

"Area Segmentation of Images Using Edge Points". IEEE Trans. PAMI-2 (1980) 1, pp. 8-15.

"Extracting and Labeling Boundary Segments in Natural Scenes".

IEEE Trans. PAMI-2 (1980) 1, pp. 16-27.

"Object Enhancement and Extraction".

in: B. Lipkin, A. Rosenfeld (Eds.).

'Picture Processing and Psychopictorics'. Academic Press. 1970, pp. 75-149

"A Microprocessor-Controlled Photo-Diode

1113/ Pugh, A. Waddon, $K$. Sensor for the Detection of Gross Defects". Proc. 3rd Int. Conf. on Automated Inspection and Product Control, Nottingham, UK, Apri1 1978, pp. 299-312. 
1114/ Restrick III, R.C. "An Automatic Optical Printed Circuit Inspection System".

Proc. SPIE Vol. 116 'Solid State Imaging Devices', 1977, pp. 76-81

/115/ Ridler, T.W.

Calvard, S.

"Picture Thresholding Using an Iterative

Selection Method".

IEEE Trans. SMC-8 (1978) 8, pp. 630-632

1116/ Ringshauser, $H . \quad$ "Digitale Bildsensoren für industrielle Anwendungen in Sichtprüfung, Handhabung, Ablaufsteuerung und Prozeßregelung".

LEITZ-Symposium "Quantitative Bildauswertung und Mikroskopphotometrie, Wetzlar, Sept. 79, Sonderheft MICROSCOPICA ACTA, Hirzel Verlag, Stuttgart, 1980, pp. 298-302.

/117/ Riseman, E.M Arbib, M.A.

1118/ Riseman, E.M. Hanson, A.R.

1119/ Roberts, L.G.

/120/ Robinson, G.S. Reis, J.J.

/121/ Robinson, G.S.

1122/ Rosen, C. Nitzan, D. et a1.

/123/ Rosen, C.A.
"Computational Techniques in the Visual Segmentation of Static Scenes". Computer Graphics and Image Processing 6 (1977) pp. $221-276$

"Segmentation of Natural Scenes".

in: HANSON \& RISEMAN (Eds.): 'Computer Vision Systems', Academic Press, N.Y. 1978, pp. 129-163

"Machine Perception of Three-Dimensional Solids." in: J.Tipett, D. Berkowitz, L. Clapp, C. Koester, $\&$ A. Vanderbrugh (Eds.),

Optical and Electro-optical Information,

M.I.T. Press, 1965, pp. 159-197

"A Real-Time Edge Processing Unit".

Proc. of IEEE Workshop on 'Picture Data Description and Management', Chicago, $\Pi 1$. , U.S.A., Apri1 1977, pp. 155-164.

"Detection and Coding of Edges Using Directional Masks".

Opt. Engr. 16 (1977) 6, pp. 580-585

"Exploratory Research in Advanced Automation".

5th Report, Stanford Research Institute,

Menlo Park, CA, USA, Jan. 1976

"Machine Vision and Robotics:

Industrial Requirements".

in: 'Computer Vision and Sensor-Based Robots'

G.G. Dodd \& L. Rossol (Eds.), Plenum Press,

N.Y., 1979, pp. 3-20 
1124/ Rosenfeld, A. Thurston, $M$.

/125/ Rosenfeld, A. Kak, A.

/126/ Rosenfe1d, A.

1127/ Rosenfeld, A. Humme1, Zucker, S.

/128/ Saraga, P. Skoyles, D. R.

/129/ Schärf, R.

/130/ Shapiro, L.G.

/131/ Slansky, J.

/132/ Shirai, Y.

1133/ Spur, G. Kraft, H. -R. Sinning, $H$.
"Edge and Curve Detection for Visual Scene Analysis".

IEEE Trans. Comp. C-20 (1971), pp. 562-569

"Digital Picture Processing".

Academic Press, N.Y., 176

"Interactive Methods in Image Analys is"

Proc. IEEE Conf. on Pattern Recognition \& Image Proc. Troy, N.Y.,

June 1977

pp. 14-18

"Scene Labeling by Relaxation Operations". IEEE Trans. SMC-6 (1976), pp. $420-433$

"An Experimental Visually Controlled Pick and Place Machine for Industry".

Proc. 3rd International Joint Conf. on Pattern Recognition, Coronado, CA, November 1976, pp. 17-21

"Untersuchungen zur mehrkanaligen Bildverarbeitung und objektseparierung".

Proc. 'Digital Image Processing', GI/NTG conference, March 1977, Munich, H. -H. Nagel (Ed.), Informatik-Fachberichte 8 Springer-Verlag, 1977, pp. 280-294

"A Structural Model of Shape".

CS Dept. Tech. Report CS 79003-R, Virginia Polytechnic Institute \& State Univ., Blacksburg, VA, USA April 1979

"Image Segmentation and Feature Extraction". IEEE Trans on Systems, Man, and Cybernetics SMC-8 (1978) 4, pp. 237-247.

"Recognition of Real-World Objects Using Edge Cues".

in: Hanson, A. \& E. Riseman (Eds.): 'Computer Vision Systems', Academic Press, N.Y., 1978, pp. 353-362

"Optisches Erkennungssystem mit Halbleiterbildsensoren zur Steuerung von Industrierobotern". ZwF 73 (1978) 7, pp. 363-366. 
/134/ Steusloff, H. (Ed.)

/135/ Sterling, W.M.

/136/ Stockman, G.C. Agrawala, A.K.
"Wege zu sehr forgeschrittenen Handhabungssystemen". Fachberichte Messen-Steuern-Regeln, Band 4, Springer-Verlag, Berlin, 1980

\footnotetext{
"Automatic Non-Reference Inspection of Printed Wiring Boards".

Proc. IEEE Conf. on Pattern Recognition and Image Processing, Chicago, 111., August 1979, pp. 93-100
}

"Equivalence of Hough Transformation To

Template Matching".

'Interactive Screening of Reconnaissance Imagery'

L.N.K. Corp., AMRL-TR-76-15

Silver Spring, Md

June 76

pp. 105-114

/137/ Tani, K.

Abe, $M$.

Tanie, $K$.

Ohno, T.

"High Precision Manipulator with Visual Sense". Proc. 7 th Int. Symp. on Industrial Robots, Tokyo, October 1977, pp. 561-568.

Proc. Int. Joint Conf. on Artificial Intelligence, D.A. Walker \& L.M. Norton (Eds. 1969)

pp. 521-526 a.

/139/ Thissen, F.L.A.M. "Ein Gerät für die automatische optische Kontrolle von Verbindungsleiterbahnmustern für integrierte Schaltungen".

Philips Technische Rundschau 37 (1977/78) Nr. 4, pp. 85-96.

/140/ Toda, H.

"Kawasaki Vision System -- Mode $179 A^{\prime}$.

Masaki, I.

Proc. 1oth Int. Symp. on Industrial Robots, Milan, Italy, March 1980 ,

pp. 163-174

/141/ Tokumitsu, J.

Kawata, S.

Ichioka, Y.

Suzuki, T.
"Adaptive Binarization Using A Hybrid Image Pro- cessing System".
Applied Optics 17 (1978) №. 16, Aug.,
pp. $2655-2657$


/142/ Tropf. H.

/143/ Vanderbrug, G.J. Albus, J.S. Barkmeyer, E.

/144/ Veillon, F.

1145/ Ward, M.R. Rossol. L. Holland, S.W. Dewar, R.

/146/ Wedlich, G.

$1147 /$ Willett, T.J. Bluzer, $N$.

/148/ Willett, T.J. Brooks, C.W. Tisdale, G.E.

/149/ Wolf, H.

/150/ Yachida, M. Ikeda, $M$. Tsuji, S.
"Analys is-by-Synthes is Search to Interpret Degraded Image vata.

1st International Conference on Robot Vision and Sensory Controls, Stratford-on-Avon, UK. Apri 1 1-3, 1981

"A Vision System for Real Time Control of Robots".

Proc. 9th Int. Symp. on Industrial Robots, Washington D.C., March 1979

pp. 213-231

"One Pass Computation of Morphological and Geometrical Properties of Objects in Digital Pictures".

Signal Processing 1 (1979) 3, pp.

"CONSIGHT: A Practical Vision-Based Robot Guidance System".

Proc. 9th Int. Symp. on Industrial Robots, Washington D.C., March 1979

pp. 195-211

"Serienreifes Gerät zur lokaladaptiven Videosignalverarbeitung".

IITB-Mitteilungen 1977

Fraunhofer-Gesel1 schaft, Karlsruhe, pp. $24-26$

"CCD Implementation of An Image Segmentation Algorithm".

Proc. 'Image Understanding Workshop', Science Applications,

Palo Alto, CA, USA, 1977 , pp. $9-11$.

"Relaxation, Systolic Arrays, and Universal Arrays".

Proc. Image Understanding Workshop' Palo Alto, CA, USA, April 79, pp. 164-170

"Optisches Abtastsystem zur Identifizierung und Lageerkennung dreidimensionaler Objekte".

Feinwerktechnik \& Messtechnik 87 (1979) 2, pp. 86-88.

"A Knowledge Directed Line Finder for Analysis of Complex Scenes".

Proc. IJCAI 179, Tokyo, August 79, pp. 984-991 
/151/ Zamperoni, P.

"Darstellung von Binärbildern mit $\mathrm{Hilfe}$ von dilatierten Kernen"

in: J.P. Foith (Ed.): 'Angewandte Szenenanalyse', Informatik-Fachbericht 20, Springer-Verlag, Berlin, 1978, pp. 124-128.

/152/ Zucker, S.W.

Hummel, R.A.

Rosenfeld, A.

"An Application of Relaxation Labeling to Line and Curve Enhancement".

IEEE Trans. Comp. C-26 (1977) 4, pp. 394-403

/153/ Zurcher, J.M.

"Conception D'Un Systeme De Perception Visuelle Pour Robot Industriel".

Compte rendus des Journées de Microtechnique, Ecole Polytechnique Federale, Lausanne, 1978 , pp. 175-193.

/154/ Zurcher, J.-M.

"Extraction de contours en traitment électronique des images II: Processeur spécialisé pour signal vidêo".

Bu11. ASE/UCS (Switzerland) 70 (1979) 11, 9 juin, pp. 532-536 